\title{
Nonlinear Fourier Analysis: Rogue Waves in Numerical Modeling and Data Analysis
}

\author{
Alfred R. Osborne \\ Nonlinear Waves Research Corporation, Alexandria, VA 22314, USA; alosborne@protonmail.com
}

Received: 7 March 2020; Accepted: 22 June 2020; Published: 9 December 2020

\begin{abstract}
Nonlinear Fourier Analysis (NLFA) as developed herein begins with the nonlinear Schrödinger equation in two-space and one-time dimensions (the 2+1 NLS equation). The integrability of the simpler nonlinear Schrödinger equation in one-space and one-time dimensions (1+1 NLS) is an important tool in this analysis. We demonstrate that small-time asymptotic spectral solutions of the 2+1 NLS equation can be constructed as the nonlinear superposition of many 1+1 NLS equations, each corresponding to a particular radial direction in the directional spectrum of the waves. The radial $1+1$ NLS equations interact nonlinearly with one another. We determine practical asymptotic spectral solutions of the 2+1 NLS equation that are formed from the ratio of two phase-lagged Riemann theta functions: Surprisingly this construction can be written in terms of generalizations of periodic Fourier series called (1) quasiperiodic Fourier (QPF) series and (2) almost periodic Fourier (APF) series (with appropriate limits in space and time). To simplify the discourse with regard to QPF and APF Fourier series, we call them NLF series herein. The NLF series are the solutions or approximate solutions of the nonlinear dynamics of water waves. These series are indistinguishable in many ways from the linear superposition of sine waves introduced theoretically by Paley and Weiner, and exploited experimentally and theoretically by Barber and Longuet-Higgins assuming random phases. Generally speaking NLF series do not have random phases, but instead employ phase locking. We construct the asymptotic NLF series spectral solutions of $2+1$ NLS as a linear superposition of sine waves, with particular amplitudes, frequencies and phases. Because of the phase locking the NLF basis functions consist not only of sine waves, but also of Stokes waves, breather trains, and superbreathers, all of which undergo complex pair-wise nonlinear interactions. Breather trains are known to be associated with rogue waves in solutions of nonlinear wave equations. It is remarkable that complex nonlinear dynamics can be represented as a generalized, linear superposition of sine waves. NLF series that solve nonlinear wave equations offer a significant advantage over traditional periodic Fourier series. We show how NLFA can be applied to numerically model nonlinear wave motions and to analyze experimentally measured wave data. Applications to the analysis of SINTEF wave tank data, measurements from Currituck Sound, North Carolina and to shipboard radar data taken by the U. S. Navy are discussed. The ubiquitous presence of coherent breather packets in many data sets, as analyzed by NLFA methods, has recently led to the discovery of breather turbulence in the ocean: In this case, nonlinear Fourier components occur as strongly interacting, phase locked, densely packed breather modes, in contrast to the previously held incorrect belief that ocean waves are weakly interacting sine waves.
\end{abstract}

Keywords: nonlinear Fourier analysis; nonlinear random waves; rogue waves; nonlinear wave modeling; quasiperiodic Fourier series; almost periodic Fourier series 


\section{Contents}

1. Introduction to Nonlinear Fourier Analysis

2. Overview of Linear and Nonlinear Fourier Analysis

2.1. Discussion of the Classical "Linear Model"

2.2. Overview of Linear Fourier Analysis and Its Capabilities

2.3. Development of Nonlinear Fourier Analysis

2.4. Quasiperiodic Fourier Series for Solving Nonlinear Wave Equations, Numerical Modeling and Data Analysis

2.5. Extending Quasiperiodicity to Almost Periodicity

2.6. The Sub Grid Scale Problem

3. The Nonlinear Schrödinger Equation (NLS) in Two-Spatial Dimensions

3.1. The 2+1 NLS Equation

3.2. The Modulational Dispersion Relation

3.3. Goals of This and Future Papers

4. The Nonlinear Spectrum of Two-Dimensional Wave Trains

4.1. Plane Wave Solutions of the 2+1 NLS Equation

4.2. Modulation Dispersion Relation and Nonlinearity with Rotation

5. Constructing Asymptotic Solutions of the Two-Dimensional Schrödinger Equation

5.1. NLS in 2+1 Dimensions

5.2. Computing the $2+1$ NLS Equation Asymptotic Solutions Using Riemann Theta Functions

5.3. Generalize the $2+1$ NLS Equation to Arbitrary Potential: The Hirota Method

5.4. Computation of the Nonlinear Spectrum in terms of the Riemann Matrix

5.5. Riemann Spectrum for the 1+1 NLS Equation

5.6. Riemann Spectrum for the 2+1 NLS Equation

6. The Physics of the NLFA Formulation

6.1. Overview of the Formulation

6.2. A Simple Example for a Single Degree of Freedom

7. Numerical Methods of Nonlinear Fourier Analysis

7.1. Overview of Numerical Methods for Determining the Nonlinear Spectrum in 2+1 Dimensions

7.2. Procedure for Computing the NLFA Spectrum of the Measured Surface Wave Elevation $\eta(x, y, t)$

7.3. Interpretation of Boxed Equation (106)

7.4. Quasiperiodic Fourier Structure of Measured Riemann Theta Functions

7.5. Numerical Analysis in Two Dimensions: Measurement of a Wave Field of the Sea Surface at $t=0$

7.6. Numerical Analysis for a 1D Sea Surface Elevation

7.7. Concrete Example for a Single Degree of Freedom

7.8. Alternative General Notation

7.9. Primer on Nonlinear Fourier Analysis as a Theory of Nonlinearly Interacting Stokes Waves

7.10. Modelling the Surface Wave Elevation

7.11. Computing the Frequencies $\Omega_{j}=\mathbf{n} \cdot \mathbf{\Omega}$ for Time Series Analysis and Modelling

7.11.1. The Linear Model

7.11.2. Linear Schrödinger Equation in 1+1 Dimensions

7.11.3. The Modulational Instability for $1+1$ NLS

7.11.4. LSE in $2+1$ Dimensions

7.11.5. The Modulational Instability in 2D

8. Application to Analysis of SINTEF Wave Tank Data

9. Application to Analysis of Wave Data in Currituck Sound

10. Application to Radar Data Assimilation and Modelling, and Real Time Prediction of Ocean Waves

11. Summary and Discussion

Appendix A-What Is Fourier or Harmonic Analysis?

References

\section{Introduction to Nonlinear Fourier Analysis}

Nonlinear Fourier analysis (NLFA) is a relatively new field of mathematical physics, numerical analysis, and space/time series analysis, which offers a number of capabilities for working with nonlinear wave motion not offered by traditional periodic Fourier analysis. Many useful properties of NLFA arise 
because of a recent theoretical breakthrough that allows one to transform nonlinear wave motion into a linear superposition of sine waves (Osborne [1-5]). This very surprising and seemingly paradoxical result can be obtained only by generalizing the definition of Fourier series, as used in the field of harmonic analysis: Here, one leverages quasiperiodic Fourier series (see Samoilenko [6] and cited references) and almost periodic Fourier series (see Corduneanu [7] and cited references) to describe nonlinear wave motion. Astronomers have used quasiperiodic Fourier series for over 150 years to work with fundamental problems in celestial mechanics (see Delaunay [8], Poincaré [9-11]). The modern theory of Hamiltonian chaotic dynamics of Kolmogorov-Arnol'd-Moser (KAM) theory (see Kolmogorov [12], Arnold [13-15]) is based on quasiperiodic Fourier series. Furthermore, the integrability of nonlinear wave equations depends on quasiperiodic Fourier series also (Baker [16], Mumford [17,18], Kotljarov and Its [19], Previato [20], Tracy and Chen [21], Osborne [1-5]). This is the first paper in the fields of oceanography and ocean engineering that attempts to use quasiperiodic Fourier series to model nonlinear waves and to analyze data. Of particular importance has been the development of methods for large degrees of freedom, say 1000 to 10,000 nonlinear Fourier modes. Here are a number of useful observations about NLFA:

(1) Even though NLFA is itself a linear superposition of sine waves, the Fourier parameters are not the traditional ones of standard periodic Fourier analysis. NLFA leverages quasiperiodic Fourier series to describe nonlinear waves and the QPF parameters are particular, vast in number and are obtained with the mathematics of harmonic analysis (Bohl [22], Bohr [23], Besicovitch [24], Bochner [25,26] and Jessen [27]).

(2) NLFA is applied to particular nonlinear wave equations whose exact spectral solutions are found using the inverse scattering transform (as this method is known in the United States, see [1]) or finite gap theory (as it is known in Russia, see Belokolos et al. [28]). Equations solvable by these methods are the well-known "soliton equations" such as the Korteweg-deVries (KdV), the Kadomtsev-Petviashvili (KP) and the nonlinear Schrödinger (NLS) equations. Equations of this type have "coherent structure" solutions such as Stokes waves, solitons, vortices, and breather trains. Integrable equations of this type are also known as "soliton theories." The linear superposition law in item (1) above arises from soliton theories when periodic or quasiperiodic boundary conditions hold (see Osborne [1-4]).

(3) The NLFA spectrum is a (Riemann) matrix, not a vector as in standard Fourier series. The diagonal elements of the Riemann matrix correspond to Stokes waves and the off-diagonal elements correspond to pair-wise interactions amongst the Stokes waves. NLFA is therefore a theory of Fourier analysis with Stokes wave basis functions. This contrasts to standard Fourier analysis, which is a theory of non-interacting sine wave basis functions. The dynamics of a nonlinear random wave train with many Stokes waves in the spectrum can be described only by quasiperiodic Fourier series, not by standard periodic Fourier series.

(4) The nonlinearity parameter of NLFA, called the Benjamin-Feir $(B F)$ instability parameter $\left(I_{B F}\right)$, is proportional to the wave steepness divided by the spectral bandwidth (Osborne [1]):

$$
I_{B F}=\frac{2 \sqrt{2} k_{o}^{2} a_{0}}{K}
$$

where $a_{0}$ is the carrier amplitude, $k_{0}$ is the carrier wave number and $K$ is the modulational wavenumber. Large steepness $k_{o} a_{0}$ and small bandwidth $K / k_{o}$ give larger BF parameter and therefore greater nonlinearity, leading to breather trains.

(5) When the BF parameter is much smaller than 1, the NLFA components are sine waves. When the BF parameter is larger, but still less than 1, the components are sine waves and Stokes waves. When the BF parameter exceeds 1, two Stokes waves in the spectrum phase lock together to form a type of nonlinear beat, most often called a breather wave train. Further increase of the BF parameter can lead to nonlinear spectra dominated by breather trains, often referred to as a rogue 
sea. There are also superbreather solutions, which consist of an infinite hierarchy of phase locked Stokes waves and breathers of increasing complexity. Breathers have been seen often in ocean waves, but up to now superbreathers have not been observed. Figure 1 gives a simple perspective of the typical ocean wave spectrum found by NLFA when all three types of Fourier modes are active in a particular energetic sea state.

(6) Breathers have a coherent nonlinear dynamical behavior as they do not undergo linear dispersion as occurs in a linear packet, but instead maintain their packet shape during their evolution while their amplitudes oscillate up and down (they "breathe"), i.e., they are coherent structures. Breathers are a well-known source of rogue or freak waves in oceanic sea states [1,5]. Breather packets are single nonlinear Fourier modes in the NLFA spectrum of ocean waves and are "coherent structures" that mathematically correspond to "hyperbolic vortices" in the coherent turbulence of ocean waves (Osborne et al. [5]), just as "elliptic vortices" appear in classical 2D fluid turbulence. A breather can rise up to as much as three or four times the height of the background waves. The evolution of typical breather is shown in Figure 2.

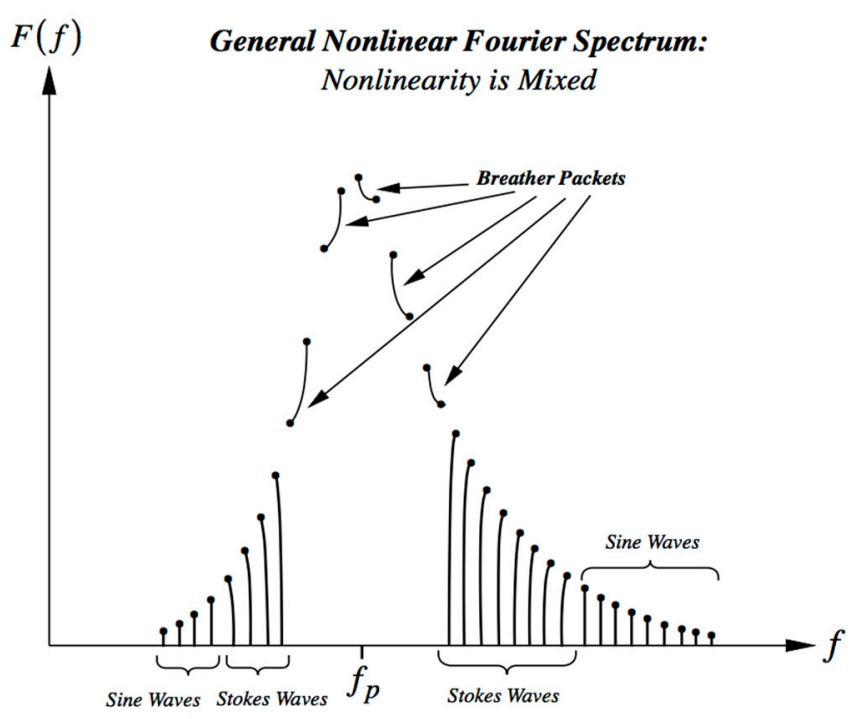

Figure 1. A typical nonlinear ocean wave spectrum has sine waves (in the right- and left-hand tails), Stokes waves (at intermediate amplitudes) and breathers (clustered about the peak of the spectrum). The black dots are "simple point eigenvalues" and the black lines are "spines" (Osborne [1]). When the spines connect an eigenvalue to the frequency axis one has sine or Stokes wave components (Osborne et al. [5]). When one has spines that directly connect two points of spectrum above the frequency axis, one has a breather component. The large peak of the spectrum indicates high nonlinearity (large BF parameter), and is dominated by five breathers.

There are several advantages of NLFA over traditional Fourier analysis for science and engineering purposes. Many of the useful properties arise because of the linear superposition law that they obey. Here is a list of a few of them:

(1) NLFA as quasiperiodic Fourier series solve the well-known integrable nonlinear wave equations and approximately solve many others (Belokolos et al. [28], Eliasson et al. [29], Osborne [1], Osborne et al. [5]). This allows a direct approach for describing solutions of the KdV, KP, and nonlinear Schrödinger equations. Furthermore, at higher order, the Dysthe [30], Trulsen-Dysthe [31], and Zakharov [32,33] equations can be addressed using these series. At higher order still, the Zakharov equation can be studied with such series. Certain aspects of higher order modulation theory have been addressed by Kimmoun et al. [34]. 
(2) NLFA can be used to nonlinearly Fourier analyze space/time series [1,5]. In this way, one determines the nonlinear (Riemann) spectrum from measured time series in terms of sine waves, Stokes waves, and breathers.

(3) NLFA can be used for the computation of the directional spectra of measured space/time series and wave fields. This means that sine waves, Stokes waves, and breathers can have a range of directions in the directional spectrum.

(4) NLFA can be used to develop nonlinear numerical models of wave fields [1]. These are the first class of numerical models that naturally maintain the coherent structures of nonlinear waves (Stokes waves and breathers) in the formulation. The numerical methods compute these coherent structures, which are nonlinear Fourier modes, as a matter of course in the numerical model.

(5) Since NLFA is a linear superposition of sine waves, one can introduce a nonlinear random phase approximation by using random phases in the quasiperiodic Fourier series (Osborne [2-4] and Osborne et al. [5]). NLFA is different than the traditional random phase approximation because phase locking allows the formation of Stokes waves and breathers in the nonlinear spectrum. In this way, the nonlinear Fourier series are then viewed as nonlinear random processes. One can then compute correlation functions, power spectra, triple correlations, coherence functions, transfer functions, and many other properties of these stochastic processes analytically, even though they are fundamentally nonlinear (Osborne [1-5]).

(6) One can analytically compute particle velocity kinematics under wave fields using quasiperiodic Fourier series. This leads to the idea that nonlinear transfer functions can be computed from quasiperiodic Fourier series, thus connecting wave amplitudes and particle velocity components.

(7) Wave forces based upon Morison's equation and diffraction theory can be computed analytically using NLFA. This allows nonlinear Fourier computations of wave forces on offshore structures, ships, breakwaters, etc. with NLFA.

(8) Wind/Wave modeling can be improved with NLFA. This allows the specific implementation of rogue waves into forecasting/hindcasting models. Up to now, rogue waves have not been reliably predicted in forecasting models.

(9) Real-time wave data assimilation onboard ships can be accomplished with NLFA.

Indeed, any application of traditional periodic Fourier series in ocean engineering can only now be carried over to quasiperiodic Fourier series for nonlinear water wave dynamics. Such is the power of the linear superposition of sine waves, even for nonlinear problems.

Herein, we address three uses of NLFA among all of the applications given above, primarily to limit the length of this paper:

(1) The NLFA analysis of surface wave data from the long wave flume at SINTEF.

(2) Analysis of measured time series data from Currituck Sound, North Carolina, USA.

(3) The radar measurements and modeling of two-dimensional wave fields carried out by the United States Navy.

The following arguments are covered in the remainder of this paper. In Section 2, we give an overview and comparison between standard Fourier analysis and nonlinear Fourier analysis. In Section 3, we discuss the $2+1$ nonlinear Schrödinger (NLS) equation and its properties. These properties are important for the development of nonlinear Fourier methods. Section 4 discusses how the solutions of the $2+1$ NLS equation can be viewed in terms of a directional spectrum whose physics along any radial direction in the spectrum is the solution of the one-dimensional $1+1$ NLS equation. This result suggests that solutions of the two-dimensional NLS equation can be constructed by nonlinear interactions of these one-dimensional solutions. Section 5 discusses how to construct the nonlinear "asymptotic" solutions for $2+1$ NLS by nonlinearly interacting the one-dimensional radial solutions of the equation. This, formally, is the mathematical structure of NLFA. To make all of this clear, in Section 6, we discuss the physical interpretation of the NLFA formulation in terms of the modulational instability for the 
simplest cases. Section 7 gives a brisk but important overview of the numerical methods of NLFA. In Section 8, we give an overview of the analysis of wave data from the long wave flume at SINTEF. In Section 9, we discuss the NLFA of data from Currituck Sound, North Carolina. Finally, in Section 10, we give a brief overview of how to analyze radar data in real time on board Naval vessels. Section 11 gives a summary and discussion of the results.
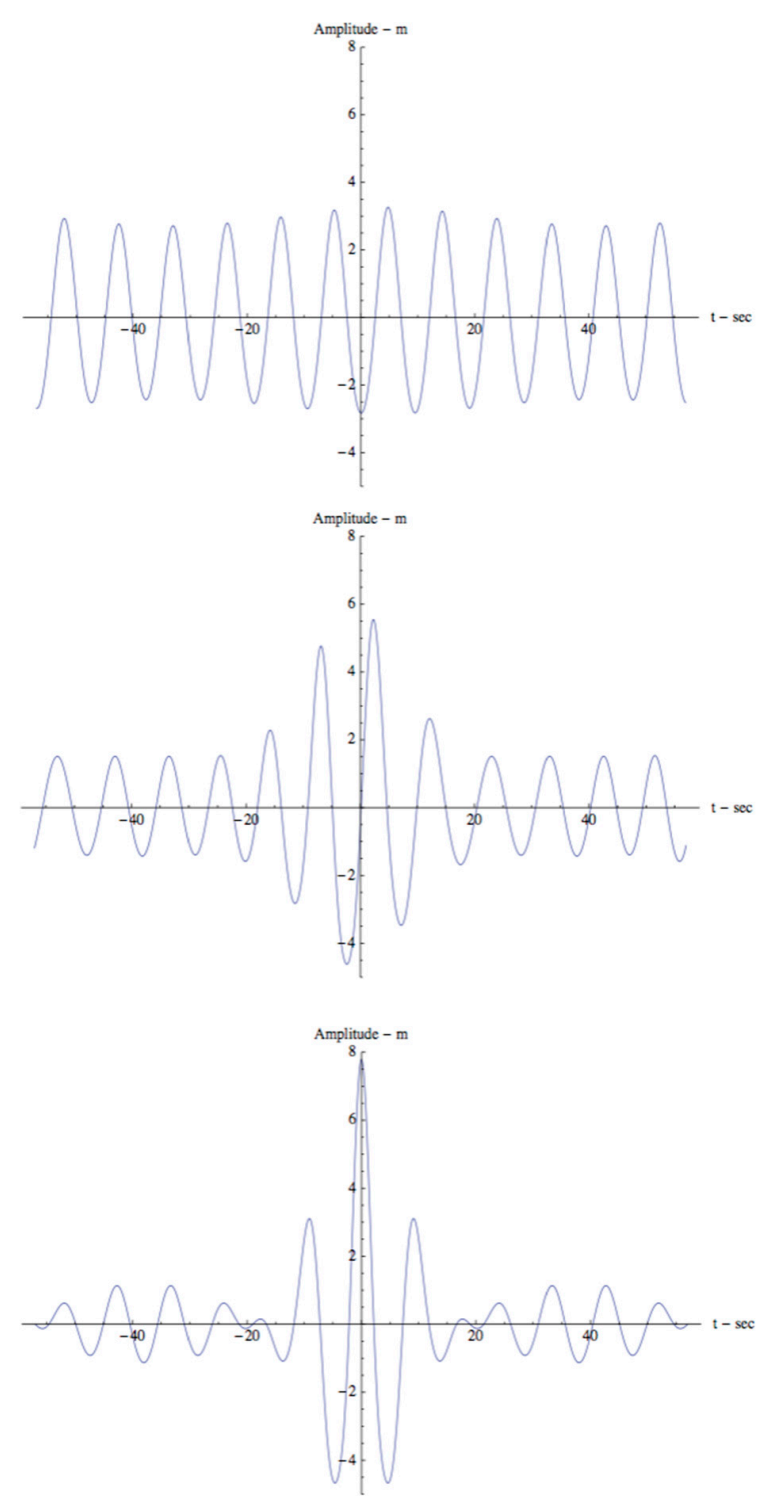

Figure 2. Evolution of a breather packet, or nonlinear beat. A small amplitude modulation of a $3 \mathrm{~m}$ sine wave (upper panel) evolves over two kilometers to form a well-defined wave packet (central panel). After a total of four kilometers the wave train evolves into a robust, localized wave packet with a maximum amplitude of $8 \mathrm{~m}$ (lower panel). The evolution from a low amplitude sine wave to a large amplitude packet is due to the Benjamin-Feir instability. This maximal state generates a central wave that is $8 / 3=2.67$ times the initial amplitude. This behavior has tempted many investigators to refer to the central wave as a "rogue wave." After the full localization of the wave packet in the lower panel, one finds that the packet then begins decreasing in amplitude once again and returns to the initial state in the upper panel, in exact reverse order. Such a dynamical motion is one "breath" of the nonlinear "dynamical breathing" of the modulational instability and constitutes a "breather cycle time." See Osborne et al. [5] for more details. 


\section{Overview of Linear and Nonlinear Fourier Analysis}

\subsection{Discussion of the Classical "Linear Model"}

We are very much used to applying a linear superposition law for describing many features of ocean waves. The idea of a "linear model" occurred during the 1940s and was first proposed by Barber et al. [35], and Longuet-Higgins and Barber [36] (see also Longuet-Higgins [37,38]), based on the seminal work of Paley and Weiner [39] on almost periodic Fourier series (see Appendix A for further discussion). The fundamental idea is the following. Ocean waves are described in two space dimensions and time by a complex Fourier series of the form:

$$
\zeta(x, y, t)=\eta(x, y, t)+i \tilde{\eta}(x, y, t)=\sum_{n} \zeta_{n} e^{i k_{n} x+i l_{n} y-i \omega_{n} t+i \phi_{n}}
$$

Formally speaking, (1) is an "almost periodic Fourier series" such that is there is no requirement for commensurable (equally spaced) wavenumbers and frequencies. The real part of this Fourier series is the surface elevation

$$
\eta(x, y, t)=\sum_{n} \eta_{n} \cos \left(k_{n} x+l_{n} y-\omega_{n} t+\phi_{n}\right)
$$

The imaginary part of (1), which we call the auxiliary surface elevation, is the Hilbert transform of (2a):

$$
\tilde{\eta}(x, y, t)=\sum_{n} \eta_{n} \sin \left(k_{n} x+l_{n} y-\omega_{n} t+\phi_{n}\right)
$$

This latter condition guarantees analyticity of (1) in the complex plane (see Osborne [1]). One assumes that the wavenumbers $(k, l)$ are dense in the $k, l$ plane: This means that there are an infinite number of wavenumbers in an infinitesimally small area $d k d l$. The $\phi_{n}$ are assumed to be uniformly distributed random numbers on $(0,2 \pi)$; in this way, (1) may be viewed as a stochastic process. The $\omega_{n}$ are the frequencies, formally assumed to be a function of the wavenumbers in terms of:

$$
\omega_{n}^{2}=\omega_{n}^{2}\left(k_{n}, l_{n}\right)=g \kappa_{n} \tanh \kappa_{n} h, \quad \kappa_{n}=\sqrt{k_{n}^{2}+l_{n}^{2}}
$$

We have here used the depth-dependent linear dispersion relation (3), where $g$ is the acceleration of gravity and $h$ is the water depth. Below we will modify (3) by: (a) increasing it to nonlinear order and (b) introducing the effects of the modulational instability (see Benjamin and Feir [40]). An important property of the components $\omega_{n}$ is that they are incommensurable even if the wavenumbers $k_{n}, l_{n}$ are chosen to be commensurable, a key feature which we exploit in this paper. In any infinitesimal element of the wavenumber plane $d k d l$, the Fourier amplitudes $\eta_{n}$ obey the relation:

$$
\frac{1}{2} \sum_{d k d l} \eta_{n}^{2}=E(k, l) d k d l
$$

Thus, we see that $E(k, l) d k d l$ is the contribution to the mean square wave height made by wave components in the infinitesimal wavenumber box $d k d l$. Here, $E(k, l)$ is the two-dimensional wave spectrum. The mean square wave height is then given by:

$$
\left\langle\eta^{2}\right\rangle=\underbrace{\lim }_{L_{x}, L_{y}, T \rightarrow \infty} \frac{1}{8 L_{x} L_{y} T} \int_{-L_{x}}^{L_{x}} \int_{L_{y}}^{L_{y}} \int_{T}^{T} \eta^{2}(x, y, t) d x d y d t=\frac{1}{2} \sum_{n} \eta_{n}^{2}=\int_{-\infty}^{\infty} \int_{-\infty}^{\infty} E(u, v) d u d v
$$

The definition (5), used by Longuet-Higgins [41], is based upon the definition of an almost periodic Fourier series (APF) (see Paley and Weiner [39], Corduneanu [7], and cited references): Thus, the limits in (5) imply that there is no periodicity, but at some intermediate value of the limiting process (for fixed 
$\left.L_{x}, L_{y}, T\right)$ the series (1) is quasiperiodic. The almost periodic limit occurs as the spatial and temporal lengths $L_{x}, L_{y}, T$ tend to infinity. Thus, $\eta(x, y, t)$ is considered to be a stationary stochastic process, where the Fourier series (1) is its spectral representation and $E(k, l)$ is its power spectrum. Stochastic processes of this type have been described in detail by Doob [42] and Papoulis [43]. It is worth noting that, in the book by Paley and Weiner [39], no periodic constraints were applied for the wavenumbers and frequencies, and the phases are not necessarily random: This is the generalized perspective taken in the present paper.

In light of the generality of (1) as an almost periodic Fourier series with a high density of incommensurable spectral components in the wavenumber and frequency domains, it is surprising that subsequent use of (1) has been reduced most commonly to standard periodic Fourier series with commensurable wavenumbers and frequencies. In models and in data analysis, we are used to applying the fast Fourier transform (FFT) with commensurable wavenumbers and frequencies. A major perspective of this paper is the idea that (1) can still be used to solve nonlinear wave equations by using incommensurable wavenumbers and frequencies, by leveraging a nonlinear form of the dispersion relation and by particular choices for the phases. We will see how this perspective works out below. Frankly, it is quite shocking that linear Fourier series like (1) can be used to solve nonlinear wave equations, as discussed in recent papers by Osborne [2-4]. We exploit these ideas for modeling and data analysis.

Of course, we are all familiar with the measurement of ocean waves by a single probe at, say $x=y=0$, so that $(2 \mathrm{a})$ becomes:

$$
\eta(t)=\sum_{n} \eta_{n} \cos \left(\omega_{n} t-\phi_{n}\right)
$$

Furthermore,

$$
\frac{1}{2} \sum_{d \omega} \eta_{n}^{2}=E(\omega) d \omega
$$

Here, $E(\omega)$ is most often referred to as the energy spectrum of $\eta(t)$. Clearly, $E(\omega)$ is the cosine transform of the correlation function used by G. I. Taylor:

$$
C(\tau)=\underbrace{\lim }_{T \rightarrow \infty} \int_{-T}^{T} \eta(t) \eta(t+\tau) d t
$$

This formulation has been given a rigorous foundation by Khintchine [44]. Rice [45] used (6), again with uniformly distributed random phases to find the statistical properties of $\eta(t)$. As shown below, the above steps (1)-(8), for the classical "linear model," will also be applicable to the "nonlinear model" of NLFA (anticipating Equation (9) below).

\subsection{Overview of Linear Fourier Analysis and Its Capabilities}

The fields of physical oceanography and ocean engineering use the standard periodic Fourier series (1) in a very pragmatic way. By putting (1) in discrete form (for indeed space/time series are discrete), one finds the discrete inverse Fourier transform by simply changing the sign of the exponential. Then, the fast Fourier transform (FFT) allows (1), and its inverse, to be computed rapidly. Linear random waves are addressed simply by using uniformly distributed random phases in (1), i.e., the so-called random phase approximation. In this case, (1) can be used to compute all kinds of important properties of waves. Indeed, one introduces the correlation function, power spectrum, coherence functions, etc. to characterize the random stochastic properties of random waves. Then, (1) becomes the basis of stochastic kinetic equations to characterize slowly varying sine waves in sea states driven by the wind. The present paper addresses a question: Is there a similar formulation that can allow us to conveniently determine the full properties of nonlinear coherent wave trains (nonlinear Fourier 
decompositions of Stokes waves, breathers and superbreathers) in the same spirit as linear wave trains are described by (1)? One possibility for carrying out such a plan is Equation (9) below, which is the main topic of this paper.

\subsection{Development of Nonlinear Fourier Analysis}

The goal of this paper is to discuss how generalizations of standard Fourier series, referred to as quasiperiodic (Samoilenko [6]) and almost periodic Fourier series (Paley and Weiner [39], Corduneanu [7]), can be constructed to replace the linear superposition law (1) for solving nonlinear wave motion. Both quasiperiodic and almost periodic Fourier series are generalized Fourier series that are linear superpositions of sine waves, but at the same time they solve nonlinear wave equations. This idea, that linear superpositions solve nonlinear wave dynamics problems, may seem like a miracle, but instead it is just rigorous mathematics. In recent papers, it was found that this approach solves by generalized Fourier series nonlinear equations such as the exactly integrable Korteweg-deVries (KdV), Kadomtsev-Petviashvili (KP) and one-dimensional nonlinear Schrödinger (1+1 NLS) equations (Osborne [2-4], and Osborne et al. [5]). Here the asymptotic quasiperiodic and almost periodic Fourier series solutions of the nonlinear Schrödinger equation $(2+1 \mathrm{NLS})$ are constructed, which describes two-dimensional wave motions in deep water, and whose solutions can be viewed as a nonlinear stochastic process in the same sense that (1) is a stochastic process. This approach is referred to as the nonlinear random phase approximation (Osborne [1-4], Osborne et al. [5]).

Basically, this means that nonlinear water waves can be described as a linear superposition of sine waves (1), but with a generalized definition for the Fourier series and its parameters. In this way, (1) can be written as a Fourier series with Stokes wave basis functions, plus phase locked Stokes waves that form breather states, the latter a mathematical pathway to one type of rogue wave. The nonlinear basis functions interact nonlinearly with each other. We refer to this formulation as nonlinear Fourier analysis (NLFA) with Stokes wave and breather basis functions. The miracle of this method is that nonlinear wave motion can be represented as a linear superposition of sine waves. A more detailed mathematical discussion is given in Appendix A.

\subsection{Quasiperiodic Fourier Series for Solving Nonlinear Wave Equations, Numerical Modeling, and Data Analysis}

Now we make a bold leap for solving nonlinear wave equations and instead of (1) we use quasiperiodic Fourier (QPF) series that have the form:

$$
\zeta(x, y, t)=\sum_{\mathbf{n} \in \mathbb{Z}^{N}} \zeta_{\mathbf{n}} e^{i \mathbf{n} \cdot \mathbf{k} x+i \mathbf{n} \cdot \mathbf{l} y-i \mathbf{n} \cdot \boldsymbol{\omega} t+i \mathbf{n} \cdot \boldsymbol{\phi}}
$$

Here, $\mathbf{n}$ is an $N$-dimensional integer summation vector $\mathbf{n}=\left[n_{1}, n_{2} \ldots n_{N}\right], \mathbf{k}, \mathbf{l}$ are $N$-dimensional wavenumber vectors so that $\mathbf{n} \cdot \mathbf{k}=\mathbf{n} \cdot\left[k_{1}, k_{2} \ldots k_{N}\right], \mathbf{n} \cdot \mathbf{l}=\mathbf{n} \cdot\left[l_{1}, l_{2} \ldots l_{N}\right]$ and $\mathbf{n} \cdot \boldsymbol{\omega}=\mathbf{n} \cdot \boldsymbol{\omega}(\mathbf{n} \cdot \mathbf{k})=$ $\mathbf{n} \cdot\left[\omega_{1}, \omega_{2} \ldots \omega_{N}\right]$ is an $N$-dimensional frequency vector (as determined by the nonlinear dispersion relation of a particular nonlinear wave equation (Osborne [2]), and $\boldsymbol{\phi}$ is an $\mathrm{N}$-dimensional phase vector $\mathbf{n} \cdot \boldsymbol{\phi}=\mathbf{n} \cdot\left[\phi_{1}, \phi_{2} \ldots \phi_{N}\right]$. The "dot" or "." indicates the inner or dot product:

$$
\mathbf{n} \cdot \mathbf{k}=\left[n_{1}, n_{2} \ldots n_{N}\right] \cdot\left[k_{1}, k_{2} \ldots k_{N}\right]=\sum_{i=1}^{N} n_{i} k_{i}
$$

Equation (9) is the basis for much of the NLFA formulation of nonlinear waves. One finds that (9) solves all nonlinear, integrable wave equations (Osborne [1-4]). We see that such a formulation is also linear, but due to the dot product in (9), the formulation contains all harmonics to all orders for all of the Fourier components. With these harmonics, one is able to construct all of the coherent waveforms that occur in integrable nonlinear wave equations. These include the Stokes waves, solitons, breathers, superbreathers, vortices, etc. that are the Fourier modes called coherent structures in the motion of 
nonlinear waves. We also suggest herein that (9) asymptotically solves higher order equations such as those due to Dysthe [30] and Trulsen-Dysthe [31].

One goal of the present paper is to address the possibility that (9) can be used to characterize ocean waves to determine their nonlinear properties just as (1) determines properties of linear waves. Of course, (1) has been extremely useful in the study of ocean waves over the past 70 years. It may well be that (9) will become even more useful over the next 70 years.

\subsection{Extending Quasiperiodicity to Almost Periodicity}

One further observation is that we can set in (9) the following changes of variable (Osborne [1]):

$$
\begin{gathered}
k_{n} \leftrightarrow \mathbf{n} \cdot \mathbf{k}=\mathbf{n} \cdot\left[k_{1}, k_{2} \ldots k_{N}\right] \\
l_{n} \leftrightarrow \mathbf{n} \cdot \mathbf{l}=\mathbf{n} \cdot\left[l_{1}, l_{2} \ldots l_{N}\right] \\
\omega_{n} \leftrightarrow \mathbf{n} \cdot \mathbf{\omega}=\mathbf{n} \cdot \mathbf{\omega}(\mathbf{n} \cdot \mathbf{k})=\mathbf{n} \cdot\left[\omega_{1}, \omega_{2} \ldots \omega_{N}\right] \\
\phi_{n} \leftrightarrow \mathbf{n} \cdot \boldsymbol{\phi}=\mathbf{n} \cdot\left[\phi_{1}, \phi_{2} \ldots \phi_{N}\right] \\
\zeta_{n} \leftrightarrow \zeta_{\mathbf{n}} \\
n \leftrightarrow \mathbf{n} \in \mathbb{Z}^{N}
\end{gathered}
$$

In this new notation, formally speaking, (9) looks like (1).

We have therefore reduced the construction of quasiperiodic Fourier series (9) to almost periodic Fourier series (1), a surprising result. Thus, we have an exceptional conclusion: The "nonlinear model for solving nonlinear, integrable wave equations" (9) is a linear superposition of sine waves. Formally speaking then (9) is equivalent to the "linear model" (1), also a linear superposition of sine waves. Therefore, we are essentially solving nonlinear partial differential equations with linear tools similar to those we use to solve linear equations.

For the nonlinear case, however, the wavenumbers and frequencies are generally incommensurable and the frequencies and phases must include nonlinear effects in the wave train, including the Stokes wave (which requires phase locking) and the modulational instability (use of the modulational dispersion relation together with phase locking). We therefore have a bigger job determining the coefficients of (9), i.e., the nonlinear Fourier amplitudes, wavenumbers, frequencies, and phases are not the same as those in (1). We therefore must learn a new art for computing the parameters of the quasiperiodic Fourier series for solving nonlinear problems, for numerical modeling and for analyzing data. The major goal of this paper is to provide a number of ways to do this and to apply the method to data analysis and modeling.

\subsection{The Sub Grid Scale Problem}

In the applications of the linear model (1), we formally assume that the wavenumbers are dense in any wavenumber box $d k d l$. However, when we actually carry out numerical computations, we choose particular small intervals for the smallest wavenumbers $d k, d l$. The lack of information inside these small wavenumber intervals is often referred to as the "sub grid scale problem." On the other hand, when we actually carry out numerical computations using quasiperiodic Fourier series (9), these small intervals are actually "filled in densely" with the harmonics $\mathbf{n} \cdot \mathbf{k}$. Therefore, in the context of nonlinear Fourier analysis, the small grid scale problem is worked by assuming quasiperiodicity so that the smallest interval resolutions $d k, d l, d \omega$ are filled in by the harmonics $\mathbf{n} \cdot \mathbf{k}, \mathbf{n} \cdot \mathbf{l}, \mathbf{n} \cdot \mathbf{w}$ : In this way, the actual "delta wavenumbers and frequencies" are numerically on the order of $10^{-6}$ of the resolutions chosen in typical wave models (Osborne [1]). To better understand this fact, note that the wavenumbers and frequencies are typically chosen to be commensurable (equally spaced) in standard Fourier analysis, as can be seen here:

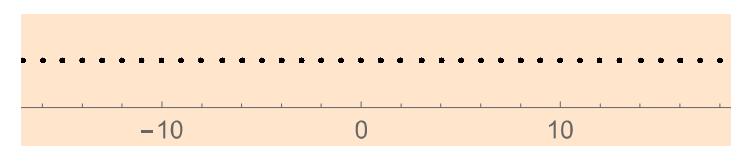


We call the associated Fourier spectrum a "discrete spectrum." However, because the dispersion relation is nonlinear the frequencies $\boldsymbol{\omega}$ are incommensurable so that the actual quasiperiodic frequencies $\mathbf{n} \cdot \boldsymbol{\omega}$ look like the following set of points:

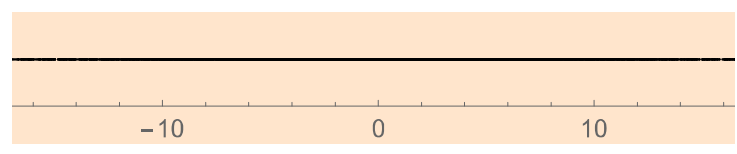

We can see that the $\mathbf{n} \cdot \boldsymbol{\omega}$ form a dense set of points, the so-called harmonics, so dense that, for practical purposes, they seem continuous. Such a point set is called a discretuum because the points are formally discrete, but are so dense that they seem by the naked eye to be continuous (discrete + continuous $=$ discretuum). Thus, the actual frequencies fill in the gaps between the discrete points of standard Fourier analysis. For incommensurable wavenumbers, the $\mathbf{n} \cdot \mathbf{k}, \mathbf{n} \cdot \mathbf{l}$ also form a dense set of points in $d k d l$ as required in the Longuet-Higgins (and Paley and Weiner) formulation of surface waves (9), formally and mathematically an almost periodic Fourier series.

The small $10^{-6}$ resolution, say, in wavenumbers and frequencies extends down to the level of the Diophantine repulsion in the Kolmogorov-Arnold-Moser (KAM) theory [6,13-15,23,29] for nonlinear Hamiltonian chaotic dynamical systems. The Diophantine condition allows integrability or quasi integrability in nonlinear wave equations, and guarantees convergence in quasiperiodic Fourier series (9). This condition resolves, for most purposes, the sub grid scale problem, a major accomplishment of the method of quasiperiodic Fourier series. Diophantine repulsion is illustrated in Figure 3.

The Diophantine Condition for Nonresonant, Quasiperiodic Motion

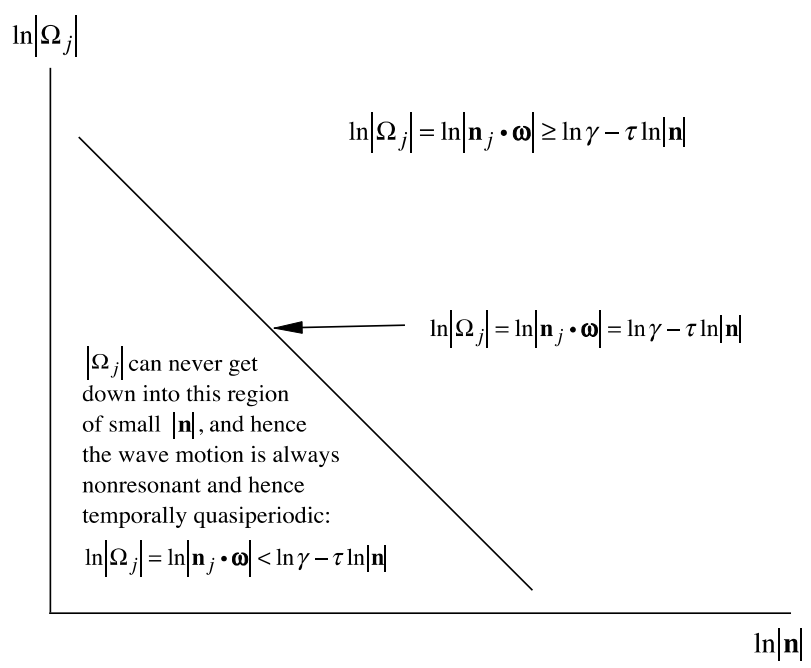

Figure 3. The smallest incommensurable frequencies in water waves $\Omega_{j}=\mathbf{n}_{j} \cdot \boldsymbol{\omega}$ in a nonlinear wave equation can never be so small that they create singularities, i.e., $\Omega_{j}=\mathbf{n}_{j} \cdot \boldsymbol{\omega} \neq 0$ for all $\mathbf{n} \neq 0$ and $\boldsymbol{\omega}$. This occurs thanks to a "geometric repulsion" that pushes $\Omega_{j}=\mathbf{n}_{j} \cdot \boldsymbol{\omega}$ away from the zero state. Such a consequence of nonlinearity is important because it guarantees that the quasiperiodic series (9), and their integrals with respect to time $t$, converge, a requirement referred to as the Diophantine condition, $\left|\Omega_{j}\right|=\left|\mathbf{n}_{j} \cdot \boldsymbol{\omega}\right| \geq \gamma|\mathbf{n}|^{-r}$ in KAM theory, for $\mathbf{n} \neq 0 \in \mathbb{Z}^{N}$ and $|\mathbf{n}|=\sum_{i=1}^{N}\left|n_{i}\right|$. The repulsion occurs below the diagonal line in the figure at very small frequencies $\left|\Omega_{j}\right|$ and small moduli of the summation indices $|\mathbf{n}|$.

The fact that the fields of physical oceanography and ocean engineering have discarded the generality of quasiperiodic and almost periodic Fourier series over the past 80 years by using finite 
discrete, commensurable frequencies/wavenumbers is a tragedy. Indeed, the often cited book by Paley and Weiner [39] has been forgotten and the application of quasiperiodic and almost periodic Fourier series to data analysis and modeling has been abandoned in favor of discrete periodic Fourier series and the fast Fourier transform. In retrospect, the generality possible in Fourier analysis for working nonlinear problems has been lost to history. This paper makes an effort to correct this situation. Appendix A gives a comparative discussion of the different methods of Fourier analysis.

\section{The Nonlinear Schrödinger Equation (NLS) in Two-Spatial Dimensions}

\subsection{The $2+1$ NLS Equation}

The NLS equation in 2+1 dimensions is given for deep water by Zakharov [32], Saffman and Yuen [46], and Yuen and Lake [47]:

$$
i\left(\psi_{t}+C_{g} \psi_{x}\right)-\mu \psi_{x x}+\delta \psi_{y y}-v|\psi|^{2} \psi=0
$$

with coefficients

$$
C_{g}=\frac{\omega_{o}}{2 k_{o}}, \quad \mu=\frac{\omega_{o}}{8 k_{o}^{2}}, \quad \delta=\frac{\omega_{o}}{4 k_{o}^{2}}=2 \mu, \quad v=\frac{1}{2} \omega_{0} k_{o}^{2}
$$

Here $\omega_{0}$ is the carrier frequency and $k_{0}$ is the carrier wavenumber. The free surface elevation has the form

$$
\eta(x, y, t)=\psi(x, y, t) e^{i k_{0} x-i \omega_{0} t}+\text { c.c. }
$$

If one is interested in the more general coefficients, which are functions of the water depth $h$, see Hasimoto and Ono [48], Mei [49], and Osborne [1]. The 2+1 NLS equation can also be written:

$$
i\left(\psi_{t}+C_{g} \psi_{x}\right)-\mu\left(\psi_{x x}-2 \psi_{y y}\right)-v|\psi|^{2} \psi=0
$$

The $2+1$ NLS equation reduces to the $1+1$ NLS equation when the $y$ term is neglected:

$$
i\left(\psi_{t}+C_{g} \psi_{x}\right)-\mu \psi_{x x}-v|\psi|^{2} \psi=0
$$

For simple scaling, together with a Galilean transformation, we have

$$
u=\rho \psi, X=x-C_{g} t, T=\mu t, \quad Y=y
$$

where

$$
\rho=\sqrt{\frac{\nu}{2 \mu}}=\sqrt{2} k_{o}^{2}
$$

We then obtain the rescaled $2+1$ NLS equation often used for theoretical studies:

$$
i u_{T}+u_{X X}-2 u_{Y Y}+2|u|^{2} u=0
$$

\subsection{The Modulational Dispersion Relation}

This subsection refers to Figure 4, which is helpful in interpreting the physics of the modulational instability. Consider the small-amplitude, modulated plane wave:

$$
\eta(x, y, t) \simeq a_{o} \underbrace{\left[1+\varepsilon \cos \left(K_{x} x+K_{y} y-\Omega t\right)\right]}_{\text {Small amplitude modulation }} \underbrace{\cos \left(k_{o} x-\left(\omega_{o}+\omega^{\prime}\right) t\right)}_{\begin{array}{c}
\text { Small amplitude } \\
\text { Stokes wave }
\end{array}}
$$


where $a_{0}$ is the carrier wave amplitude and $k_{0}$ is the carrier wavenumber and $\omega_{0}$ is the carrier frequency. Here $\omega^{\prime}$ is the Stokes wave frequency correction. The quantity in square brackets is the small amplitude modulation and the last cosine term is the carrier wave. How can we match up (19) with solutions of the $2+1$ NLS equation? How can we determine the modulational dispersion relation? This is done by noticing that the plane wave solution of NLS

$$
\psi(x, y, t)=a_{0} e^{\frac{i}{2} k_{o}^{2} a_{o}^{2} \omega_{0} t}
$$

when modified by the small amplitude modulation

$$
1+\varepsilon_{ \pm} e^{\left[i \Omega t \pm i\left(K_{x} x+K_{y} y\right)\right]}
$$

gives

$$
\psi(x, y, t)=a_{o}\left\{a+\varepsilon_{ \pm} e^{\left[i \Omega t \pm i\left(K_{x} x+K_{y} y\right)\right]}\right\} e^{\frac{i}{2} k_{0}^{2} a_{0}^{2} \omega_{0} t}
$$

Note the Stokes frequency correction in (20), (22) is given by $\omega^{\prime}=i k_{0}^{2} a_{0}^{2} \omega_{0} / 2$. Insert Equation (22) into the 2+1 NLS equation and linearize (see Yuen and Lake [47], Osborne [1]) to find the perturbation dispersion relation

$$
\Omega^{2}=\frac{\omega_{o}^{2}}{8 k_{o}^{2}}\left(\frac{K_{x}^{2}}{8 k_{o}^{2}}-\frac{K_{y}^{2}}{4 k_{o}^{2}}-k_{o}^{2} a_{o}^{2}\right)\left(K_{x}^{2}-2 K_{y}^{2}\right)
$$

which can be written in scaled form as

$$
\frac{2 \Omega}{\omega_{0} k_{o}^{2} a_{o}^{2}}=2\left(\frac{\kappa}{\sqrt{2}}\right) \sqrt{1-\left(\frac{\kappa}{\sqrt{2}}\right)^{2}}, \kappa=\sqrt{\kappa_{x}^{2}-2 \kappa_{y}^{2}}=\kappa_{x} \sqrt{1-2\left(\frac{\kappa_{y}}{\kappa_{x}}\right)^{2}}
$$

where the normalized wave numbers are

$$
\kappa_{x}=\frac{K_{x}}{2 k_{o}^{2} a_{o}} ; \quad \kappa_{y}=\frac{K_{y}}{2 k_{o}^{2} a_{o}}
$$

It is useful in what follows to apply the rescaled version of the modulational dispersion relation

$$
\frac{2 \Omega}{\omega_{o} k_{o}^{2} a_{o}^{2}}=2\left(\sqrt{\frac{\kappa_{x}^{2}-2 \kappa_{y}^{2}}{2}}\right) \sqrt{1-\left(\sqrt{\frac{\kappa_{x}^{2}-2 \kappa_{y}^{2}}{2}}\right)^{2}}
$$

We now graph the boundaries of instability. These occur

(1) When the first parenthesis in (26) is identically zero:

$$
\kappa_{x}= \pm \sqrt{2} \kappa_{y} \text { or } \kappa_{y}= \pm \frac{\kappa_{x}}{\sqrt{2}}
$$

These are two straight lines crossing at the origin (Figure 4). This means that the lines $\kappa_{y}= \pm \kappa_{x} / \sqrt{2}$ make an angle with the origin

$$
\tan \theta_{o}=\frac{\kappa_{y}}{\kappa_{x}}=\frac{1}{\sqrt{2}}
$$

where $\theta_{0}=35.2644^{\circ}$. In the wavenumber domain (again see Figure 4 ), the instability region lies between the angles $\pm 35.2644^{\circ}$.

(2) The dispersion relation is again zero when the square root on the right-hand side of (26) is zero

$$
\kappa_{x}^{2}-2 \kappa_{y}^{2}=2 \text { or } \kappa_{y}= \pm \sqrt{\frac{\kappa_{x}^{2}-2}{2}}
$$


These equations correspond to a pair of hyperbolae, concave to the right and left, shown in Figure 4. The cyan region in the spectral wavenumber domain is where the unstable modes of $2+1$ NLS occur, i.e., where breather trains form and hence where rogue waves may occur in the nonlinear Fourier spectrum.

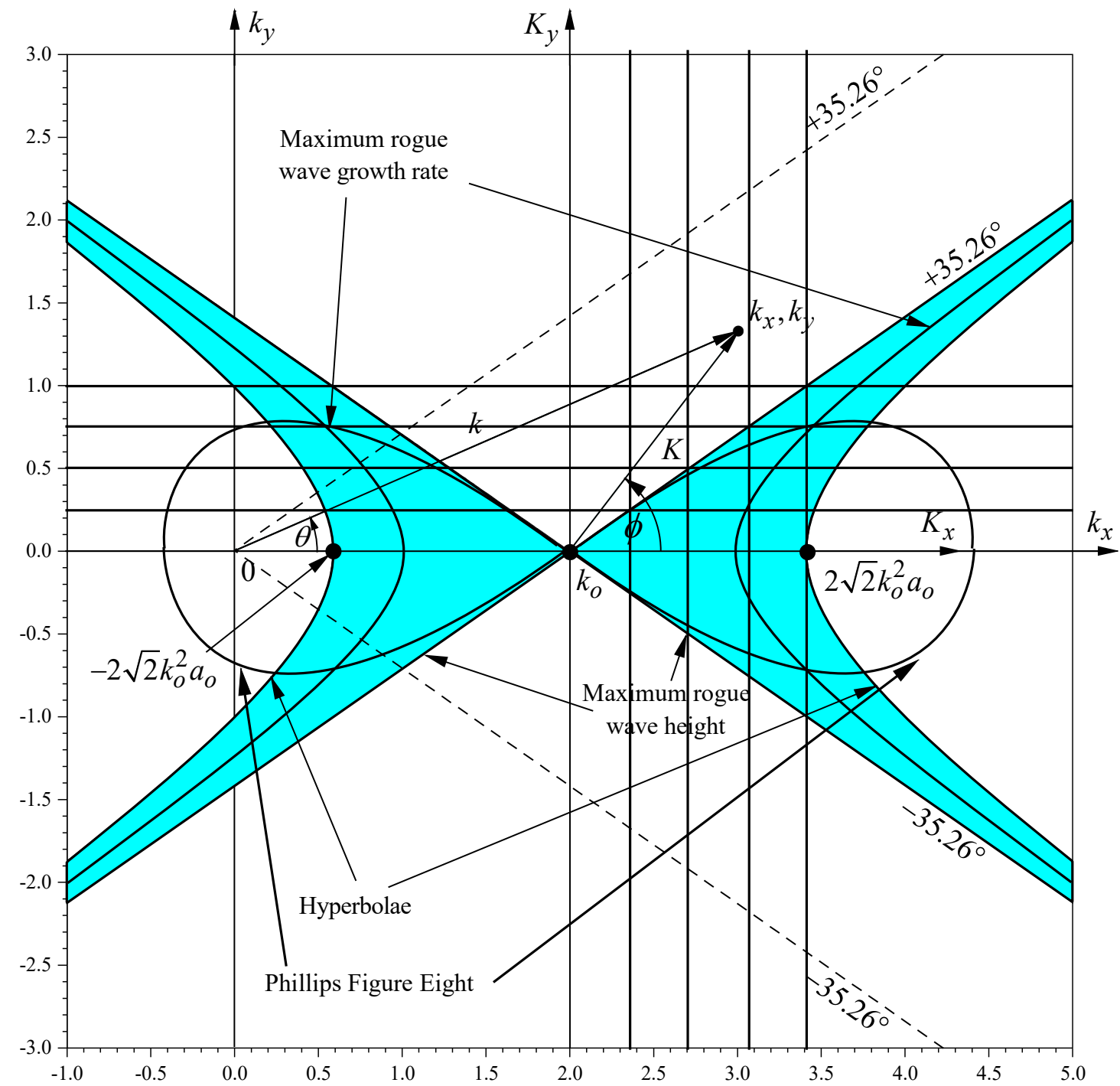

Figure 4. Boundary of modulation dispersion relation for the $2+1$ NLS equation. The cyan region is where the unstable modes occur in the spectrum (this is where the actual breathers of the quasiperiodic solutions of the equation reside). The figure eight shows where the Phillips four-wave interactions occur.

Figure 4 has been constructed from work in Longuet-Higgins [37] and Hasselmann [50]. The appearance in Figures 4 and 5 of the "Phillips Figure Eight" is a reference to the by now classical geometrical shape used to describe four-wave nonlinear resonant interactions (Phillips [51]). This figure eight is placed on Figures 4 and 5 to provide a reference for those who work with wind wave models, but who may be less familiar with deterministic models. Experimental verification of the cyan region, commonly referred to in the field as "bimodal directional spreading," has been made by Young et al [52], Ewans [53], Huang et al [54], and Long and Resio [55]. 


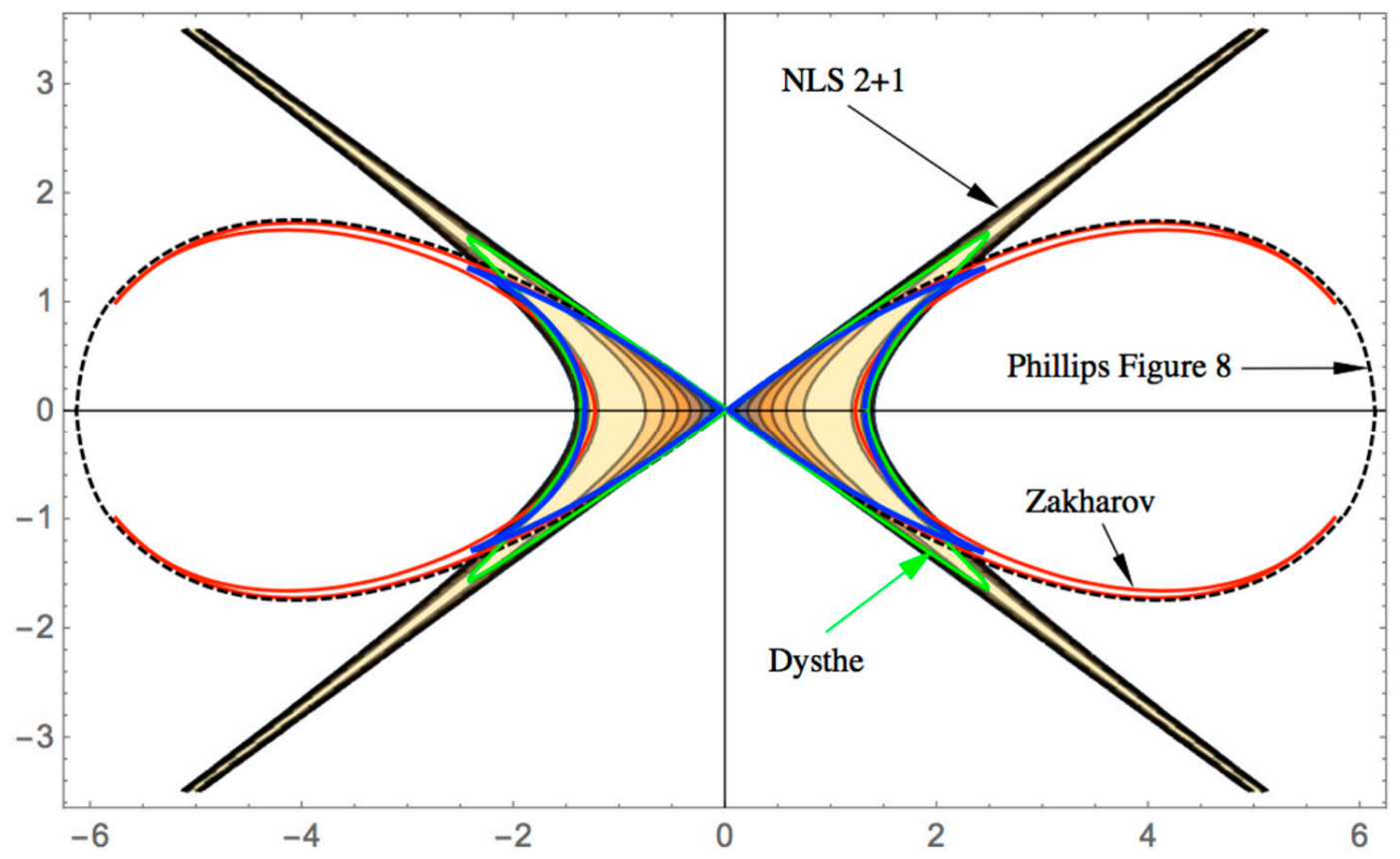

Figure 5. The wavenumber domain with the boundaries of the modulational dispersion relation for several nonlinear wave equations. The $2+1$ NLS equation boundary is shown by two crossed black lines and associated hyperbolae. The boundary of the Dysthe equation is shown in green and the boundary of the Trulsen-Dysthe equation is shown in blue. The Zakharov equation boundary is shown in red and the Phillips figure eight is represented as a dotted line. For ocean waves, the central lines cross at the peak of the directional spectrum. The horizontal wavenumber coordinate lies along the dominant direction of the wave motion and the peak has been shifted to zero as seen on the horizontal axis. Additional information about coordinate definitions is shown in Figure 4.

\subsection{Goals of This and Future Papers}

It is worthwhile discussing the less detailed but physically important representation of Figure 4, which emphasizes the nonlinear asymptotic extensions of the $2+1$ NLS equation as given in Figure 5 . The various shades of tan between the straight lines and the hyperbolae define the region of instability for the $2+1$ NLS equation. The Dysthe [30] equation provides the next order beyond the $2+1$ NLS equation, which has the instability boundary denoted by the green curve. Note that the green curve for this equation does not extend out to infinity, as does the boundary for $2+1$ NLS, but instead is limited to a finite closed region, a result that is important because it gives a more physical result. Going to a still higher order, to the Trulsen-Dysthe equation [31], one sees the instability boundary is given by the blue curve, which is more accurate still. Indeed, this latter curve is beginning to move toward the red curve, which represents the instability boundary of the Zakharov equation. The Trulsen and Dysthe [31] paper is quite excellent and develops all of these details. Note that the results for the Zakharov equation is remarkably near the Phillips figure eight, as one expects theoretically (Yuen and Lake [47]). These remarkable results have been found by exploring the regions of the Benjamin-Feir instability for the various nonlinear wave equations of interest and are fundamental to the study of NLFA as seen herein.

The modulational boundaries for the successive orders of the two-dimensional wave equations are pictographically shown as black ( $2+1$ NLS), green (Dysthe equation), and blue (Trulsen-Dysthe equation), as they asymptotically converge toward the red boundary curve (Zakharov equation) in Figure 5. It is important to recognize that any objections that one might have to the physically applicability of the $2+1$ NLS equation can be removed by going to higher order. In the remainder of this paper, we will discuss primarily how to use the boundary for the $2+1$ NLS equation (cyan region, 
Figure 4; black lines, Figure 5) to write the quasiperiodic Fourier series for the equation. Sequel papers will extend these boundaries to higher order and construct their quasiperiodic Fourier series.

The "asymptotic solutions" as used in this paper refer to approximate solutions of nonlinear wave equations that occur on time scales that are fast with respect to the slow cascading time scales of wind waves (Komen et al. [56]). Indeed, the spectral cascading is not included in the asymptotic solutions (quasiperiodic/almost periodic Fourier series) used here. Asymptotic solutions of the $2+1$ NLS equation are in fact general solutions of "nearby, integrable equations" that we will study separately in a later paper and need not be treated here.

\section{The Nonlinear Spectrum of Two-Dimensional Wave Trains}

In order to construct quasiperiodic Fourier series for the $2+1$ NLS equation, we will need a number of new results that are useful for understanding the nonlinear (so-called Riemann) spectrum of the wave motion. This section begins that process of learning. Subsequent sections will be dedicated to completing this picture.

We now discuss how solutions of the 1+1 NLS equation are also plane wave solutions of the $2+1$ NLS equation. Each plane wave corresponds to different angle and therefore solutions of $2+1$ NLS correspond to the nonlinear superposition of solutions of the $1+1 \mathrm{NLS}$ equation for each and every direction in the directional spectrum. Since we already know about the theta function solutions of $1+1$ NLS equation behave (Osborne [1]), we must only learn how these one-dimension equations interact with each other to get an estimate of the asymptotic solutions of the 2+1 NLS equation. Of course, these solutions will be in terms of quasiperiodic and almost periodic Fourier series, thus forming the basis of NLFA.

\subsection{Plane Wave Solutions of the 2+1 NLS Equation}

The challenge in the present and later sections is to obtain a class of asymptotic 2D spectral solutions of the $2+1$ NLS equation. To this end, we first seek the following useful result.

Fact: All solutions of the 1+1 NLS equation are also plane wave solutions of the 2+1 NLS equation.

This can be seen in Figure 6 where the contours of a typical spectrum are shown in the wave number plane $(k, l)=\left(k_{x}, k_{y}\right)$. The peak of the spectrum is at $(k, l)=\left(k_{x}, k_{y}\right)=\left(k_{p}, 0\right)$. The contours represent the spectrum whose dominant direction is along the $k=k_{x}$ axis. The most important result in this section is to show that any radial line constitutes a spectral solution of the 1+1 NLS equation, Equation (15). From this point of view, the solutions of the 2+1 NLS equation are "pasted together" solutions of the $1+1$ NLS equation along each of the radial lines. Each of the $1+1$ NLS lines must then interact nonlinearly with one another to form asymptotic solutions of the $2+1$ NLS equation. To obtain a general class of $2 \mathrm{D}$ asymptotic spectral solutions of the $2+1$ NLS equation is the challenge of Section 5 .

Consider a single plane-wave modulation of the form

$$
\psi(x, y, t)=\psi\left(k_{x} x+k_{y} y, t\right)
$$

In this example, we have a small-amplitude modulation of the carrier wave, the latter of which is always in the dominant direction of the $x$-axis. There exists a unique coordinate frame with axis $X$, at an angle $\alpha$ to the $x$-axis, for which this modulation has no component in the $Y$ direction:

$$
\psi(X, Y=0,0)=\psi\left(K_{x} X, 0\right)
$$

The coordinate transformation is shown in Figure 7 and is given by

$$
\left[\begin{array}{l}
X \\
Y
\end{array}\right]=\left[\begin{array}{cc}
\cos \theta & \sin \theta \\
-\sin \theta & \cos \theta
\end{array}\right]\left[\begin{array}{l}
x \\
y
\end{array}\right]
$$




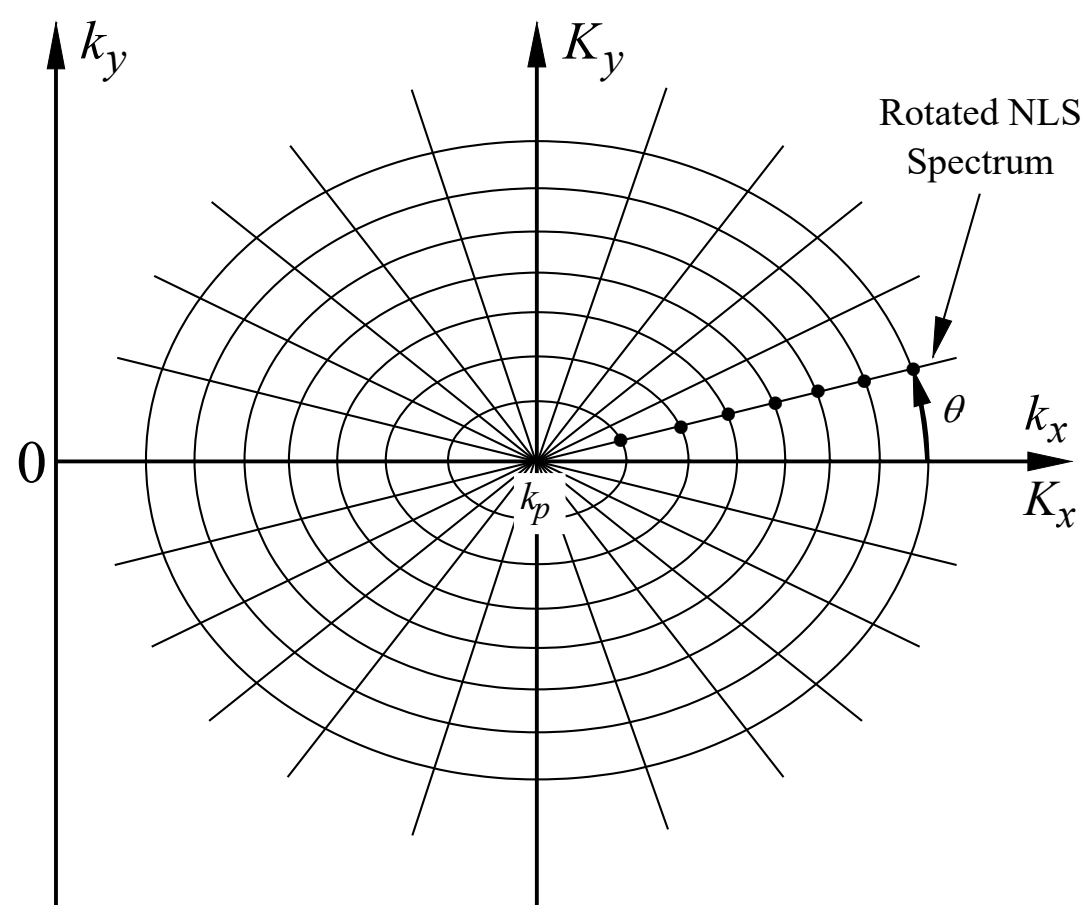

Figure 6. The wavenumber domain for the $2+1$ NLS equation $(k, l)=\left(k_{x}, k_{y}\right)$ is relative to the origin $(k, l)=\left(k_{x}, k_{y}\right)=(0,0)$. The wave spectrum is indicated by the contours: Note that the peak of the spectrum lies at $(k, l)=\left(k_{x}, k_{y}\right)=\left(k_{p}, 0\right)$ and the dominant direction lies along $k_{x}=0$. The modulational wavenumbers $\left(K_{x}, K_{y}\right)=\left(k_{x}-k_{p}, k_{y}\right)$ are centered about the peak of the spectrum. Each radial line in the spectrum is shown in the text to represent a solution of the "rotated" $1+1$ NLS equation.

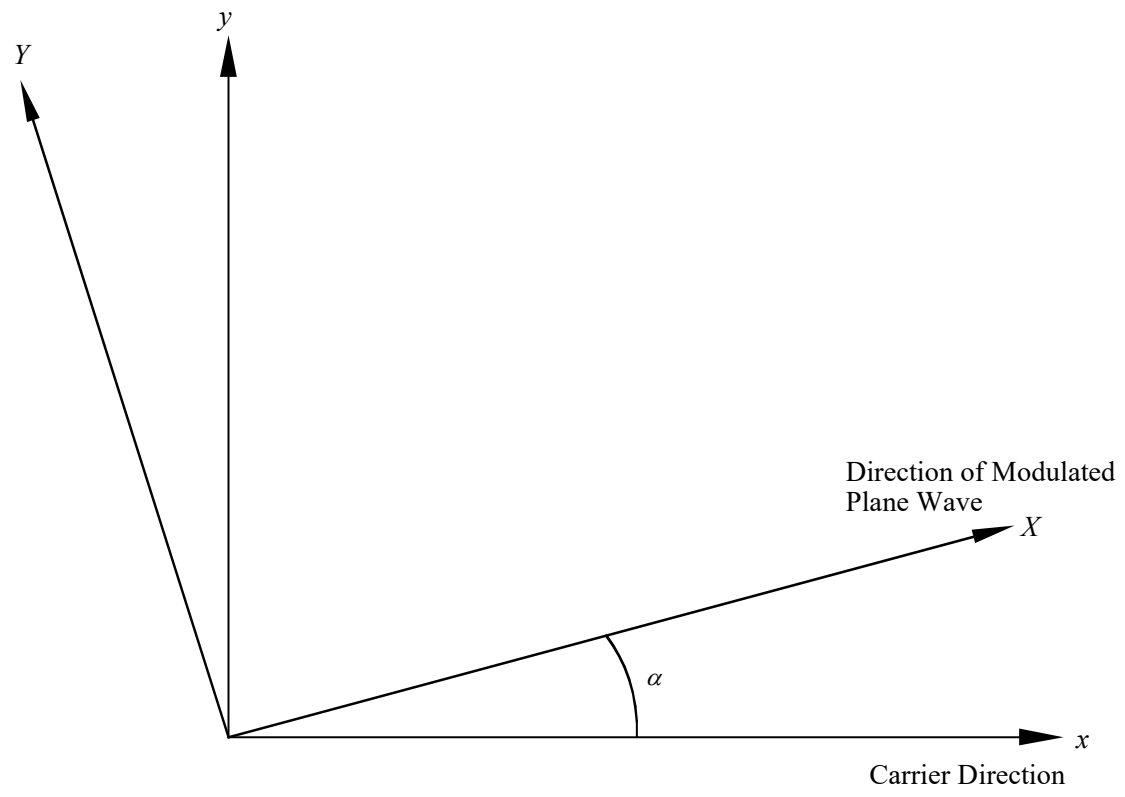

Figure 7. The carrier coordinate frame for the $2+1$ NLS equation is given by the coordinate pair $(x, y)$. The rotated coordinate frame is defined by the coordinate pair $(X, Y)$. The particular rotation angle $\alpha$ is such that $(X, Y=0)$, i.e., there is a plane wave solution of the modulation moving in the direction $X$. 
We choose $\theta=\alpha$, such that $Y=0$, and therefore the plane wave moves exactly in the $X$ direction:

$$
\left[\begin{array}{c}
X \\
0
\end{array}\right]=\left[\begin{array}{cc}
\cos \alpha & \sin \alpha \\
-\sin \alpha & \cos \alpha
\end{array}\right]\left[\begin{array}{l}
x \\
y
\end{array}\right],\left[\begin{array}{l}
x \\
y
\end{array}\right]=\left[\begin{array}{cc}
\cos \alpha & -\sin \alpha \\
\sin \alpha & \cos \alpha
\end{array}\right]\left[\begin{array}{c}
X \\
0
\end{array}\right]
$$

The inverse is

$$
\begin{gathered}
x=X \cos \alpha, \\
y=X \sin \alpha
\end{gathered}
$$

In addition

$$
\begin{aligned}
X & =x \cos \alpha+y \sin \alpha, \\
K_{x} & =k_{x} \cos \alpha+k_{y} \sin \alpha
\end{aligned}
$$

The wavenumber rotational transformation in $\left(K_{x}, K_{y}\right)$ (see Figure 6) has the form:

$$
K_{x}=K \cos \alpha, K_{y}=K \sin \alpha
$$

where the modulus of the wave number $K$ and $\tan \alpha$ have the forms:

$$
K=\sqrt{K_{x}^{2}+K_{y}^{2}}, \tan \alpha=\frac{K_{y}}{K_{x}}
$$

Another useful (hyperbolic) transformation we address below is given by:

$$
K_{x}=\kappa \cosh \phi, K_{y}=\frac{\kappa}{\sqrt{2}} \sinh \phi
$$

where

$$
\kappa^{2}=K_{x}^{2}-2 K_{y}^{2}
$$

We have already seen this structure in (24) above. Here $\kappa$ is the "hyperbolic modulus" of the hyperbolic transformation. The relationship between $\phi$ and $\alpha$ is given by:

$$
\tanh \phi=\sqrt{2} \frac{K_{y}}{K_{x}}=\sqrt{2} \tan \alpha
$$

The above rotational transformation (33) reduces the $2+1$ NLS equation to a $1+1$ NLS equation. This is seen by making the substitutions:

$$
\begin{array}{ll}
\frac{\partial \psi}{\partial x}=\frac{\partial \psi}{\partial X} \frac{\partial X}{\partial x}=\cos \alpha \frac{\partial \psi}{\partial X}, & \frac{\partial^{2} \psi}{\partial x^{2}}=\cos ^{2} \alpha \frac{\partial^{2} \psi}{\partial X^{2}} \\
\frac{\partial \psi}{\partial y}=\frac{\partial \psi}{\partial X} \frac{\partial X}{\partial y}=\sin \alpha \frac{\partial \psi}{\partial X}, & \frac{\partial^{2} \psi}{\partial y^{2}}=\sin ^{2} \alpha \frac{\partial^{2} \psi}{\partial X^{2}}
\end{array}
$$

and we find the rotated $1+1$ NLS equation

$$
i\left(\psi_{t}+\frac{\omega_{o}}{2 k_{o}} \cos \alpha \psi_{X}\right)-\frac{\omega_{o}}{8 k_{o}^{2}}\left(1-3 \sin ^{2} \alpha\right) \psi_{X X}-\frac{1}{2} \omega_{o} k_{o}^{2}|\psi|^{2} \psi=0
$$

For our purposes, it is important to note that

$$
1-3 \sin ^{2} \alpha=\cos ^{2} \alpha-2 \sin ^{2} \alpha>0
$$

which means that $\tan \alpha<1 / \sqrt{2}$ and $\alpha<35.2644^{\circ}$. An angle greater than this will change the sign of the dispersive term in the $2+1$ NLS equation and so all solutions for these large angles will correspond to simple Stokes waves. Indeed, all wave components outside the cyan region are stable Stokes waves. 
The modulational instability, and hence rogue wave packets, lie between $\pm 35.2644^{\circ}$ of the dominant wave direction, and inside the right-hand/left-hand parabola in Figure 4 (again, this is the cyan colored region in the figure): Physically and mathematically, the breathers each consist of two phase locked Stokes waves as discussed in more detail in Section 5 below.

We thus see that any solution of the $1+1$ NLS equation is a solution of the $2+1$ NLS equation. A particular plane wave has the wavenumber pair $\left(K_{x}, K_{y}\right)$ as seen in Figure 6 . The angle $\alpha$ is given by

$$
\alpha=\tan ^{-1}\left(\frac{K_{y}}{K_{x}}\right)
$$

such that $Y=0$ for each plane wave. Use this value of $\alpha$ to compute the coefficients of the $1+1$ NLS equation as given in Equation (42). We study the properties of the rotated 1+1 NLS equation below.

\subsection{Modulation Dispersion Relation and Nonlinearity with Rotation}

The rotated form of the $1+1$ NLS equation can be written as

$$
i\left(\psi_{t}+C_{g}(\alpha) \psi_{X}\right)+\mu(\alpha) \psi_{X X}+v|\psi|^{2} \psi=0
$$

where

$$
C_{g}(\alpha)=C_{g o} \cos \alpha, C_{g o}=\frac{1}{2} \frac{\omega_{0}}{k_{0}}
$$

For positive $\alpha$, this means the group speed $C_{g}(\alpha)$ is reduced for the rotated solution (Figure 8 ). The coefficients of the rotated $1+1$ NLS equation are given by:

$$
\begin{gathered}
\mu(\alpha)=\left(1-3 \sin ^{2} \alpha\right) \mu_{o}=p \mu_{0}, \mu_{o}=-\frac{\omega_{o}}{8 k_{o}^{2}} p=1-3 \sin ^{2} \alpha \\
v=v_{o}, v_{o}=-\frac{1}{2} \omega_{0} k_{o}^{2}
\end{gathered}
$$

The parameter $\mu(\alpha)$ is shown in Figure 9 to be a decreasing function of rotation angle. We see that the rotated modulation dispersion relation is given by (in terms of the coefficients of the rotated $1+1$ NLS equation):

$$
\Omega=\mu(\alpha) K \sqrt{K^{2}-\frac{2 v}{\mu(\alpha)} a_{o}^{2}}=\mu(\alpha) K \sqrt{K^{2}-4 \rho^{2}(\alpha) a_{o}^{2}}, \rho(\alpha)=\sqrt{\frac{v}{2 \mu(\alpha)}}
$$

where

$$
\rho(\alpha)=\sqrt{\frac{v}{2 \mu(\alpha)}}=\sqrt{\frac{v_{o}}{2 p \mu_{o}}}=\sqrt{\frac{2}{p}} k_{o}^{2}=\frac{\sqrt{2} k_{o}^{2}}{\sqrt{1-3 \sin ^{2} \alpha}}=\frac{\rho_{o}}{\sqrt{1-3 \sin ^{2} \alpha}}, \quad \rho_{o}=\sqrt{\frac{v_{o}}{2 \mu_{o}}}
$$

This means a rotation up to $\pm 35.2644^{\circ}$ gives a value of $\rho(\alpha)$ (this is the nonlinearity parameter that the inverse scattering transform sees (Osborne [1])) that is larger, so that the $1+1$ NLS equation is more nonlinear after the rotation (Figure 10). Normally, we are used to thinking that the spectral energy falls off for directions away from the dominant direction, simply because the spectrum tends to be reduced at angles with respect to the dominant direction. However, this drop-off effect is somewhat minimized due to the fact that the nonlinear parameter is greater for increasing angles. This effectively ensures that a broader range of radial Fourier modes is unstable as the angle $\theta=\alpha$ increases, as shown in Figure 11. 


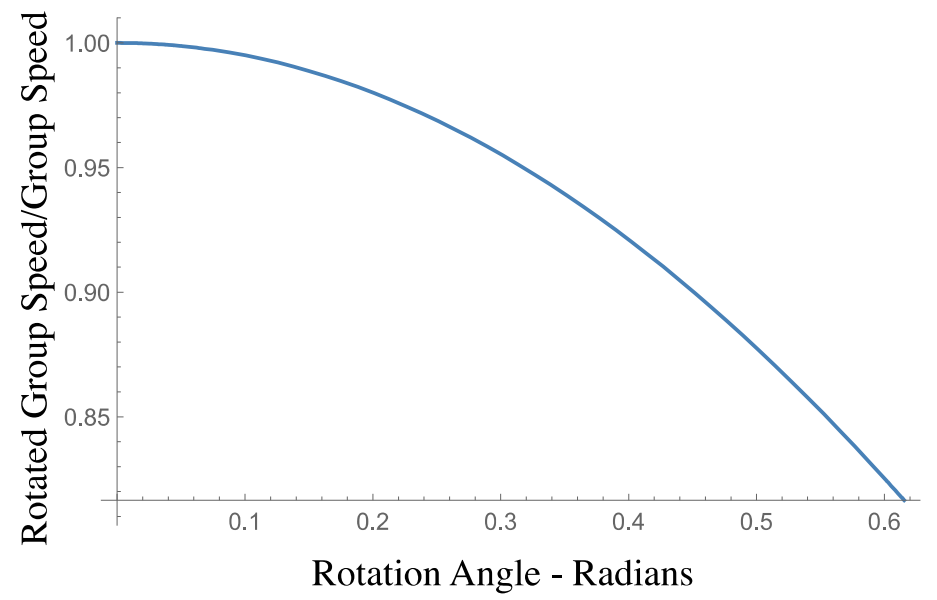

Figure 8. The group speed $C_{g}$ of the rotated $1+1$ NLS solution is reduced with respect to that for the unrotated $1+1$ NLS equation as the rotation angle varies between 0 and 35.2644 degrees.

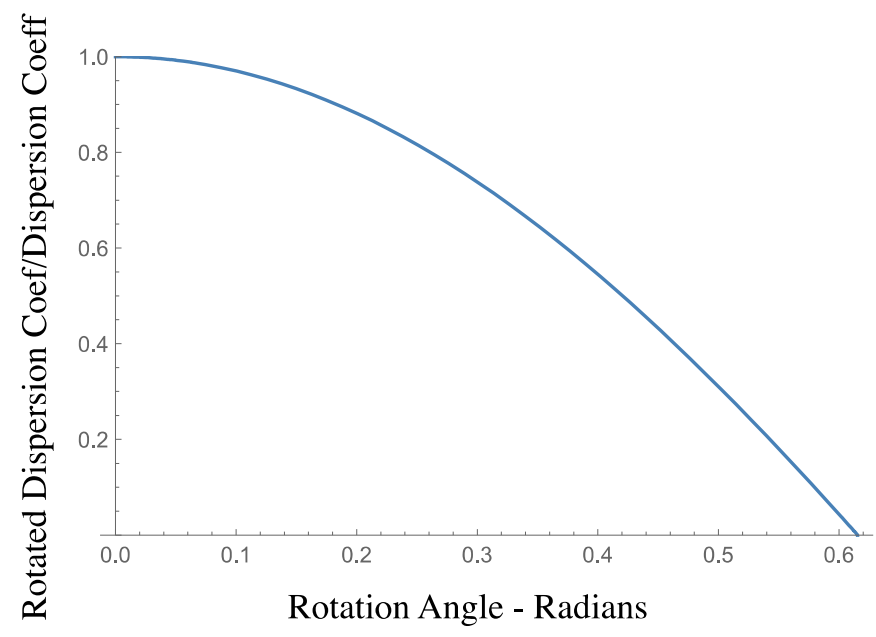

Figure 9. The dispersion coefficient $\mu(\alpha)$ of the rotated 1+1 NLS solution is reduced with respect to that for the unrotated $1+1$ NLS equation as the rotation angle varies between 0 and 35.2644 degrees.

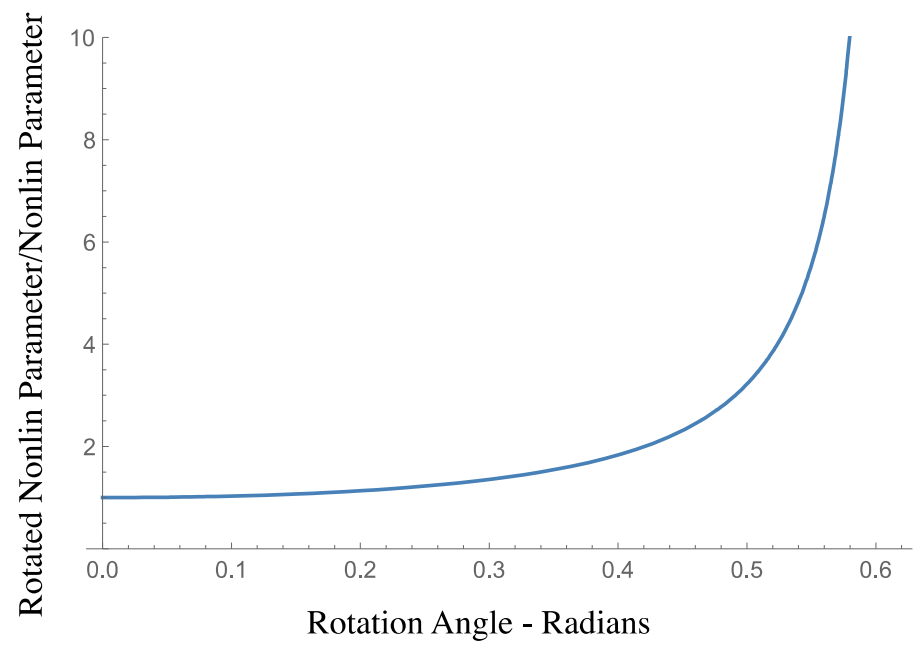

Figure 10. The nonlinearity parameter $\rho(\alpha)$ of the rotated 1+1 NLS solution is increased with respect to that for the unrotated. $1+1$ NLS equation as the rotation angle varies between 0 and 35.2644 degrees. 


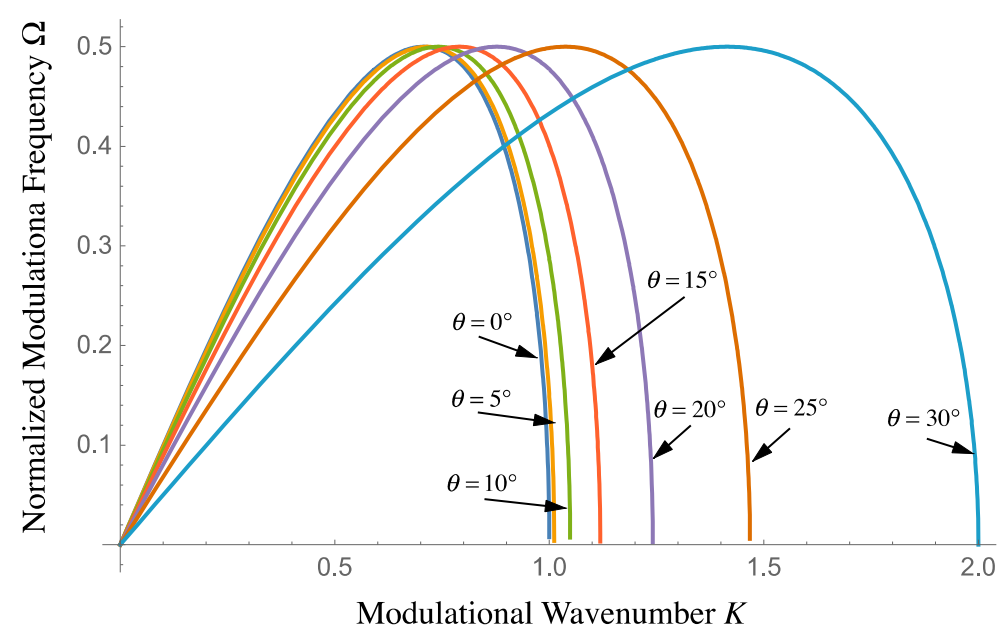

Figure 11. Instability diagram for the rotated $1+1$ NLS equation as a function of the modulational wavenumber. Several curves are given for different rotation angles. Instability diagram for the rotated $1+1$ NLS equation as a function of the modulational wavenumber and the rotation angle.

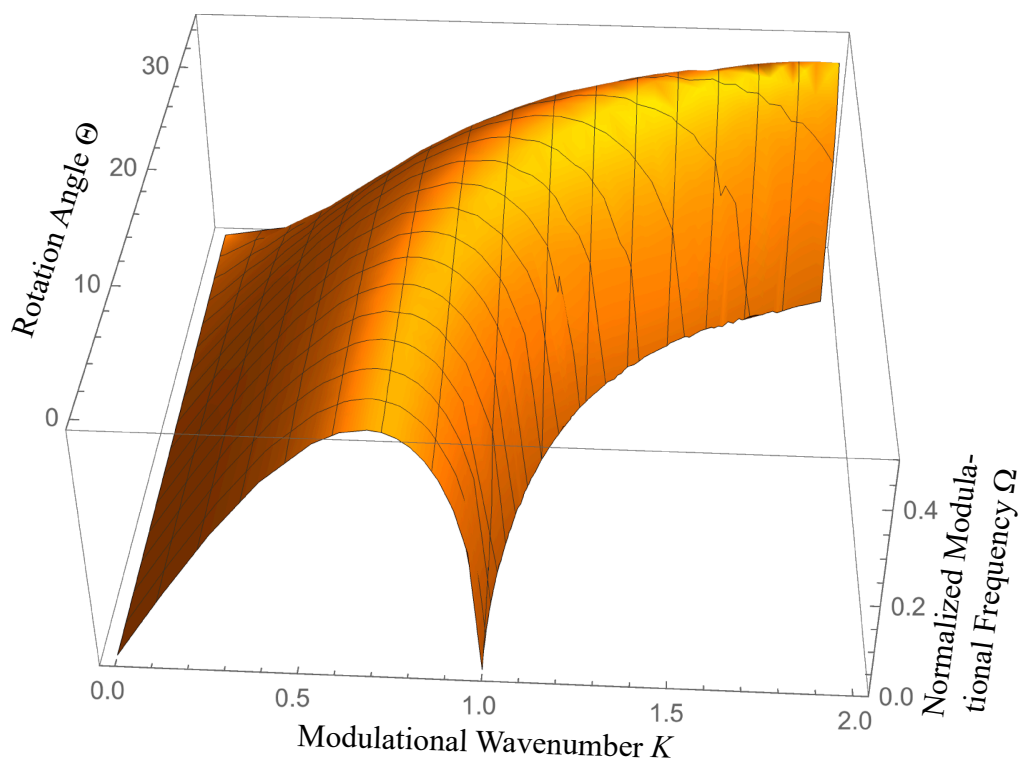

Figure 12. Instability diagram surface for the rotated $1+1$ NLS equation as a function of the modulational wavenumber and the rotation angle.

Assume that we are looking for imaginary modulation frequencies and consider the dispersion relation for unstable modes:

$$
\frac{\Omega}{\omega_{o} k_{o}^{2} a_{o}^{2}}=\left(\frac{\sqrt{p} K}{2 \sqrt{2} k_{o}^{2} a_{o}}\right) \sqrt{1-\left(\frac{\sqrt{p} K}{2 \sqrt{2} k_{o}^{2} a_{o}}\right)^{2}}
$$

This expression for the rotated modulational frequency is important and will be used to compute the Riemann matrix for $2+1$ NLS below. A graph of this rotated modulational dispersion relation, as a function of the rotation angle, is given in Figure 11. By increasing the rotation angle, we increase the width of the instability region. Figure 12 shows the surface of the modulation dispersion relation as a function of wavenumber and rotation angle. 
The rotated modulation dispersion relation in deep water then becomes:

$$
\Omega^{2}=p^{2} \frac{\omega_{o}^{2}}{8 k_{o}^{2}}\left(\frac{K^{2}}{8 k_{o}^{2}}-\frac{k_{o}^{2} a_{o}^{2}}{p}\right) K^{2}
$$

Thus, along a radial line (Figure 6) in the spectral domain $\left(K_{x}, K_{y}\right)$ of the directional spectrum, we have a larger band for instabilities and hence for rogue wave components in the spectrum.

We see that for larger angles the region of instability is broader (Figure 10), and indeed extends to infinity as the angle approaches $35.2644^{\circ}$. Physically, this means that the width of the unstable region increases with larger rotation angle up to $35.2644^{\circ}$ : Thus, for larger angles, more and more Fourier modes are unstable along the radial direction. Of course, we have already seen this in Figure 4, which occurs within the cyan region. For increasing rotation angle, the unstable region along a radial line extends to the hyperbolic bounding curve.

Now suppose that we suggest a new wavenumber definition

$$
\kappa=\sqrt{p} K=\sqrt{1-3 \sin ^{2} \alpha} K=\sqrt{K_{x}^{2}-2 K_{y}^{2}}
$$

This can be shown by using the expression

$$
1-3 \sin ^{2} \alpha=\cos ^{2} \alpha-2 \sin ^{2} \alpha
$$

Then,

$$
\kappa=K \sqrt{\cos ^{2} \alpha-2 \sin ^{2} \alpha}=\sqrt{K^{2} \cos ^{2} \alpha-2 K^{2} \sin ^{2} \alpha}=\sqrt{K_{x}^{2}-2 K_{y}^{2}}
$$

We interpret $\kappa$ as a rotated wavenumber $\kappa=\rho^{1 / 2} K$. It is seen that this is the new modulational wavenumber for rotated coordinates.

The same kind of argument leads to the fact that the maximum growth rate of a single unstable mode of the $2+1$ NLS equation, moving at an arbitrary angle with respect to the $x$-axis, as given above, is easily derived by coordinate transforming the $2+1$ NLS equation to the $1+1$ NLS equation: One finds that the maximum growth rates are invariant (remain constant), as seen in Figure 11.

Oblique breathers have been studied in the laboratory by Chabchoub et al. [57].

\section{Constructing Asymptotic Solutions of the Two-Dimensional Schrödinger Equation}

The goal of this section is to discuss general asymptotic solutions of the $2+1$ NLS equation. For our purposes, this means that we physically restrict the directional spectra to be narrowly distributed about the dominant direction and the motion of the complex envelope to be rapid with respect to the slower cascading time scale that occurs along the modulational channels towards high wavenumbers and frequencies. We now see how this is carried out.

\subsection{NLS in 2+1 Dimensions}

Recall that the nonlinear Schroedinger equation in $2+1$ dimensions is given by (11) and (12). This equation is not integrable by the inverse scattering method. In the limit that the motion becomes unidirectional, one obtains the usual one-dimensional nonlinear Schroedinger equation, which is an integrable equation. One goal of this paper is to address the possibility that "nearby" asymptotic integrability to (11) might exist and hence might also be useful for nonlinear Fourier analysis purposes. The nearby integrability will be based on the fact that rotated 1+1 NLS governs every direction in the directional spectrum of (11), but we will also have to provide nonlinear interactions between these different rotated $1+1$ NLS solutions. The simplest choice for these 2D interactions, based on 1+1 NLS, will give us "nearby integrability" and hence a structure for nonlinear Fourier analysis. 


\subsection{Computing the 2+1 NLS Equation Asymptotic Solutions Using Riemann Theta Functions}

Based on the work of Baker [16] and Mumford [17,18] (see also discussions in Osborne [2-4]), we can hypothesize an ansatz for an asymptotic solution of the $2+1$ nonlinear Schroedinger equation as the ratio of two Riemann theta functions. This is an algebraic geometric construction that was originally investigated before the 2+1 NLS equation was even known (see Matsutani [58]). The ansatz is also the exact solution of the $1+1$ NLS equation, extended to two dimensions:

$$
\psi(x, y, t)=a_{o} \frac{\theta\left(x, y, t \mid \boldsymbol{\tau}, \boldsymbol{\phi}^{-}\right)}{\theta\left(x, y, t \mid \boldsymbol{\tau}, \boldsymbol{\phi}^{+}\right)} e^{i K^{\prime} x-i \Omega^{\prime} t}
$$

The theta functions are given by

$$
\theta(x, y, t \mid \boldsymbol{\tau}, \boldsymbol{\phi})=\sum_{\mathbf{n} \in \mathbb{Z}^{N}} \theta_{\mathbf{n}} e^{i \mathbf{n} \cdot \mathbf{K}_{x} x+i \mathbf{n} \cdot \mathbf{K}_{y} y-i \mathbf{n} \cdot \mathbf{\Omega}\left(\mathbf{K}_{x}, \mathbf{K}_{y}\right) t+i \mathbf{n} \cdot \boldsymbol{\phi}}, \quad \theta_{\mathbf{n}}=e^{i \pi \mathbf{n} \cdot \tau \mathbf{n}}
$$

where $\mathbf{K}_{x}, \mathbf{K}_{y}$ are the modulational wavenumber vectors, $\mathbf{\Omega}\left(\mathbf{K}_{x}, \mathbf{K}_{y}\right)$ is the modulational dispersion relation in terms of the modulation wavenumbers, and the $\boldsymbol{\phi}$ is a phase vector. Here $\tau$ is the Riemann spectral matrix whose diagonal elements contain sine waves, Stokes waves, and breathers [1]. The off-diagonal elements provide nonlinear interactions between pairs of sine waves, Stokes wave, or breather components.

The modulation dispersion relation that appears in (57) has the following form in 2D:

$$
\Omega_{n}^{2}=\frac{\omega_{o}}{8 k_{o}^{2}}\left(\frac{K_{x n}^{2}}{8 k_{o}^{2}}-\frac{K_{y n}^{2}}{4 k_{o}^{2}}-k_{o}^{2} a_{o}^{2}\right)\left(K_{x n}^{2}-2 K_{y n}^{2}\right)+\ldots
$$

where $n=1,2 \ldots N$. Since the wavenumbers are all constant in time, because we have fixed the size of the box in which the solutions are to be determined, the leading term on the right-hand side of (58) is also constant in time. This suggests that, while formally the frequencies $\Omega_{n}$ depend on time because $2+1$ NLS is nonintegrable, temporal dependence is fairly small and occurs on a slow time scale due to the well-known cascading in the modulational channels.

The surface wave elevation associated with the asymptotic $2+1$ NLS solution is given by:

$$
\eta(x, y, t)=\operatorname{Re}\left[a_{o} \frac{\theta\left(x, y, t \mid \boldsymbol{\tau}, \boldsymbol{\phi}^{-}\right)}{\theta\left(x, y, t \mid \boldsymbol{\tau}, \boldsymbol{\phi}^{+}\right)} e^{i\left(k_{o}+K^{\prime}\right) x-i\left(\omega_{o}+\Omega^{\prime}\right) t}\right]
$$

Here $k_{o}$ and $\omega_{o}$ are the carrier wavenumber and frequency. The $K^{\prime}$ and $\Omega^{\prime}$ are the Stokes wavenumber and frequency corrections, given in Tracy and Chen [21] for the 1+1 NLS equation, and discussed experimentally in Section 7 below. A single 2D breather train solution of Equation (59), moving at $30^{\circ}$ relative to the dominant direction, for a $2 \times 2$ Riemann spectrum with locked phases (Osborne [1]), is shown in Figure 13. This is typical of the breathers that occur in the $2+1$ NLS equation which live in the peak of the spectrum in the cyan region of Figure 4. Breathers can be characterized in terms of their maximum amplitude and "rise time" to maximum amplitude (Osborne [1], Osborne et al. [5]). Breathers that move at some angle with respect to the dominant direction are similar to those that actually move in the dominant direction (one dimensional breathers). Two-dimensional breathers can be computed as a simple transformation of a one-dimensional breather by a rotation of the wavenumber $\left(\mathbf{K}_{x}, \mathbf{K}_{y}\right)$ coordinates as seen in the preceding. The nonlinear adiabatic annealing technique on a Riemann surface (NLAARS) (Osborne [51]) can be used to determine the parameters of the Riemann spectrum in Equation (59) (see also (66)) given Fourier modes $\eta_{m n}(t=0)$, i.e., given the Fourier transform of the initial condition $\eta(x, y, t=0)$. 


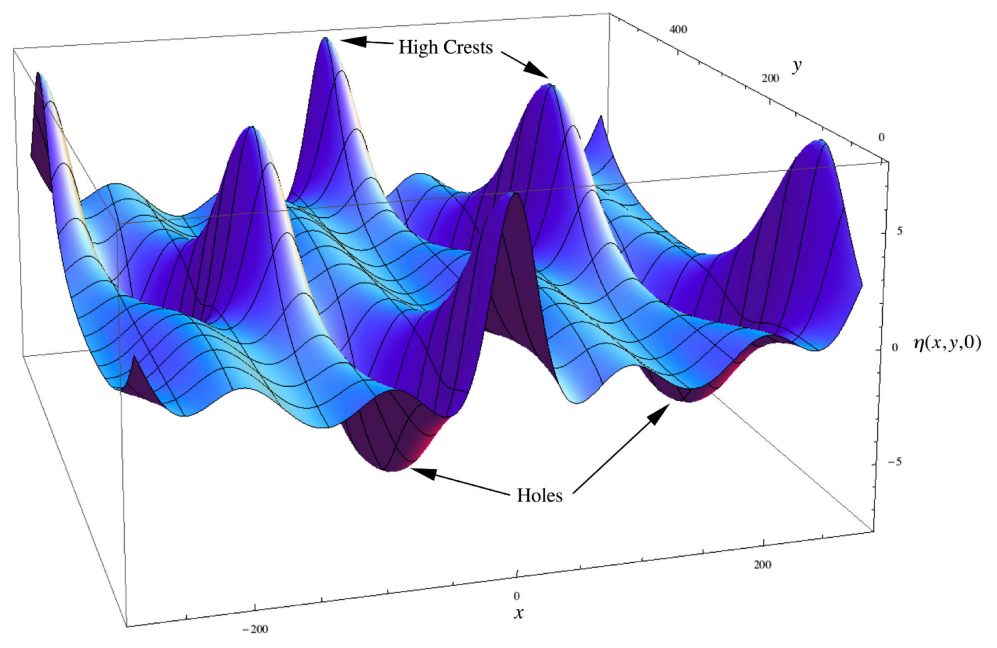

Figure 13. Oblique breather train moving at $30^{\circ}$ relative to the dominant wave direction. This is a breather wave train formed by two phase-locked Stokes waves (Equations (78)-(84) below with rotation). It is the analogue of a breather in $1+1$ dimensions and has a $2 \times 2$ Riemann spectrum from which this graph was made. This solution is a single nonlinear Fourier component in the finite gap theory for the unstable modes in the cyan region of Figure 4.

The surface wave elevation (59) arises from this simple form from the leading order term of the Stokes wave:

$$
\eta(x, y, t)=\operatorname{Re}\left[\psi(x, y, t) e^{i k_{0} x-i \omega_{0} t}\right]
$$

One can go on to as high an order that one wants to for (60), say to the Stokes fifth order approximation (Osborne [1]). Insert the above theta function solution (56) of NLS into the expression for the surface elevation (60) to get (59). Note that $a_{0}$ is the carrier wave amplitude, most often defined to be the average of the squared modulus of the complex modulation $A(x, y, t)^{2}=|\psi(x, y, t)|^{2}$ over space (see further discussion in Section 6):

$$
a_{0}^{2}=\underbrace{\lim }_{L \rightarrow \infty} \frac{1}{L^{2}} \int_{0}^{L} \int_{0}^{L}|\psi(x, y, t)|^{2} d x d y
$$

This observation suggests that small amplitude modulations play a subsidiary role in extreme ocean waves where large amplitude modulations dominate in the "rogue sea" condition in which extreme wave events dominate the sea state (Osborne et al. [1]).

It is natural to introduce a complex surface elevation by introducing the auxiliary surface elevation $\widetilde{\eta}(x, y, t)$ (the tilde indicates the Hilbert transform, Osborne [1]):

$$
\widetilde{\eta}(x, y, t)=\operatorname{Im}\left[\psi(x, y, t) e^{i k_{0} x-i \omega_{0} t}\right]=H[\eta(x, t)]
$$

where $H[$.$] is the Hilbert transform. Then, the complex surface elevation \Xi(x, t)$ is given by

$$
\Xi(x, y, t)=\eta(x, y, t)+\widetilde{\eta}(x, y, t)
$$

This leads to the connection between the complex surface elevation and the complex modulational envelope:

$$
\Xi(x, y, t)=\psi(x, y, t) e^{i k_{0} x-i \omega_{0} t}
$$


We can write (59) as a multidimensional Fourier series (Osborne [3]):

$$
\eta(x, y, t)=\sum_{\mathbf{n} \in \mathbb{Z}^{N}} \eta_{\mathbf{n}} e^{i \mathbf{n} \cdot \mathbf{k} x+i \mathbf{n} \cdot \mathbf{l} y-i \mathbf{n} \cdot \boldsymbol{\omega} t+i \mathbf{n} \cdot \boldsymbol{\phi}}
$$

The coefficients $\eta_{\mathbf{n}}$ of this Fourier series are written in terms of the theta function spectrum (Osborne [2-5]). We now write the last summation (65) as an ordinary periodic Fourier series. This requires that we assume spatially periodic boundary conditions, $\eta(x, y, t)=\eta\left(x+L_{x}, y, t\right)=\eta\left(x, y+L_{y}, t\right)$, where $L_{x}$ and $L_{y}$ are the fixed sides of the periodic box in which the wave motion occurs. Then, we obtain:

$$
\eta(x, y, t)=\sum_{m=-\infty}^{\infty} \sum_{n=-\infty}^{\infty} \eta_{m n}(t) e^{i k_{m} x+i l_{n} y}
$$

Here, as in (Osborne [1, Chapter 32]), we have:

$$
\eta_{m n}(t)=\sum_{\{\mathbf{n} \in \mathbb{Z}: I(\mathbf{n} \cdot \mathbf{K})=m, I(\mathbf{n} \cdot \mathbf{L})=n\}} \eta_{\mathbf{n}} e^{-i \mathbf{n} \cdot \boldsymbol{\omega} t+i \mathbf{n} \cdot \mathbf{\phi}}
$$

and $I($.$) is given by$

$$
I(\mathbf{n} \cdot \mathbf{k})=\frac{L}{2 \pi} \sum_{n=1}^{N} n_{n} k_{n}
$$

We see that $I(\mathbf{n} \cdot \mathbf{k})$ is a negative, zero, or positive integer, i.e., $I \in \mathbb{Z}$.

\subsection{Generalize the $2+1$ NLS Equation to an Arbitrary Potential: The Hirota Method}

We now explore how to solve the Schroedinger equation using the Hirota approach [30]. We have the $2+1$ Schrödinger equation, with complex solution $\psi(x, t)$

$$
i \psi_{t}+\mu \psi_{x x}+\sigma \delta \psi_{y y}+U(x, y, t) \psi=0
$$

This is the ordinary time-dependent Schroedinger equation in $2+1$ with an arbitrary potential function $U(x, y, t)$. Of course, (69) could be treated as the standard Schroedinger equation of quantum mechanics, but that is work outside the scope of this paper. Here we concentrate on water waves where it is best to think of the coefficients $\mu, \delta$ as being depth dependent (Hasimoto and Ono [48], Osborne [1]). Here $\sigma=-1$ for deep-water waves. Now seek a solution of (69) as the ratio of two functions (see Equation (59)):

$$
\psi(x, y, t)=\frac{G(x, y, t)}{F(x, y, t)} \simeq a_{0} \frac{\theta\left(x, y, t \mid \tau, \boldsymbol{\phi}^{-}\right)}{\theta\left(x, y, t \mid \tau, \boldsymbol{\phi}^{+}\right)} e^{i K^{\prime} x-i \Omega^{\prime} t}
$$

where $F(x, y, t) \simeq \theta\left(x, y, t \mid \boldsymbol{\tau}, \boldsymbol{\phi}^{+}\right)$and $G(x, y, t) \simeq a_{0} \theta\left(x, y, t \mid \boldsymbol{\tau}, \boldsymbol{\phi}^{-}\right) e^{i K^{\prime} x-i \Omega^{\prime} t}$. We can think of this approach as a kind of nonlinear separation of variables, as will now be demonstrated. Likewise, one can use the Baker-Mumford theorem [16-18] to assess the viability of (70) as the most general quasiperiodic, meromorphic functions from the point of view of algebraic geometry, see discussions in Osborne [2-4]. Then, assume

$$
U(x, y, t)=2 \mu \partial_{x x} \ln F(x, y, t)+2 \sigma \delta \partial_{y y} \ln F(x, y, t)+C
$$

where $C$ is an integration constant. Furthermore, we can think of the potential as having the form $U(x, y, t)=v|\psi(x, t)|^{2}+V(x, y, t)$, for some $V(x, y, t)$ that gives us the higher order wave equation that we desire, i.e., the Dysthe [30] or Trulsen-Dysthe [31] equations.

In $1 \mathrm{D},(71)$ reduces to the following expression for the $1+1$ NLS equation:

$$
v|\psi(x, t)|^{2}=2 \mu \partial_{x x} \ln \theta\left(x, y, t \mid \boldsymbol{\tau}, \boldsymbol{\phi}^{+}\right)+C
$$


a result found by Kotljarov and Its [19]. This is discussed in more detail in Section 6.

Equation (71) is a hyperbolic Poisson equation (for water waves $\sigma=-1$ ) with a source term equal to the arbitrary potential $U(x, y, t)$. The relation (71) has an arbitrary constant we interpret as the space/time mean of the potential:

$$
U(x, y, t)-\langle U(x, y, t)\rangle=2 \mu \partial_{x x} \ln F(x, y, t)+2 \sigma \delta \partial_{y y} \ln F(x, y, t)
$$

Here we have taken $\langle U(x, y, t)\rangle=C$ to be the mean of the potential $U(x, y, t)$ in space and time:

$$
\langle U(x, y, t)\rangle=\underbrace{\lim }_{L \rightarrow \infty} \frac{1}{L^{2}} \int_{0}^{L} \int_{0}^{L} U(x, y, t) d x d y
$$

Use (69)-(71) to get:

$$
i\left(F G_{t}-G F_{t}\right)+\mu\left(F G_{x x}+G F_{x x}-2 F_{x} G_{x}\right)+\sigma \rho\left(F G_{y y}+G F_{y y}-2 F_{y} G_{y}\right)=\lambda F G
$$

Then, use the Hirota operator

$$
D_{t} G \cdot F=F G_{t}-G F_{t} D_{x}^{2} G \cdot F=F G_{x x}+G F_{x x}-2 G_{x} F_{x} D_{y}^{2} G \cdot F=F G_{y y}+G F_{y y}-2 G_{y} F_{y}
$$

to write (72) in Hirota operator form:

$$
\left(i D_{t}+\mu D_{x}^{2}+\sigma \rho D_{y}^{2}\right) G \cdot F=\lambda G \cdot F
$$

The bilinear forms (71), (72) (and equivalently (74)) are generally not integrable for arbitrary potential $U(x, y, t)$.

We have taken the solution of the $2+1$ NLS equation to be the ratio of two functions $G$ and $F$. We then find two equations for obtaining these two functions given by (71) and (72). We have also associated $G$ and $F$ with theta functions of positive and negative phases (70). Inverting the quasiperiodic Fourier series, we can estimate the Riemann matrix and the two sets of phases for the plus and minus cases, the main topic of the next section. We could have motivated our choice for the ratio of the two theta functions simultaneously by Padé approximants, the Hirota method, and the Baker-Mumford theorem.

Here the Equations (71) and (72) allow us to seek an asymptotic solution of $2+1$ NLS using theta functions and quasiperiodic Fourier series. The goal is to pick a potential $U(x, y, t)$ to correspond to either the 2+1 NLS equation, the Dysthe equation, or the extended Dysthe equation: Such a form might we written $U(x, y, t)=v|\psi(x, t)|^{2}+\varepsilon V_{1}(x, y, t)+\varepsilon^{2} V_{2}(x, y, t)$, where the higher orders are obtained from the nonlinear wave equations themselves (Dysthe equation [30] and Trulsen-Dysthe equation [31]). One then computes $F(x, y, t)$ by inverting (71) (see Chapters 14-16 and 23 in Osborne [1]). Then, given $F(x, y, t)$, we solve (72) to get $G(x, y, t)$. Then, the quasiperiodic asymptotic solution to $2+1$ NLS is given by Equation (70).

Therefore, from any convenient source (numerical computation, measurement of a physical system), one obtains the complex surface elevation $\Xi(x, y, t)=\eta(x, y, t)+\widetilde{i}(x, y, t)$ for the wave motion (recall that the tilde refers to the Hilbert transform). We then compute the squared modulus $|\Xi(x, y, t)|^{2}$, and finally seek to compute the function $F(x, y, t)$ by:

$$
U(x, y, t)-C \simeq v|\Xi(x, y, t)|^{2}-v\left\langle|\Xi(x, y, t)|^{2}\right\rangle=2 \mu \partial_{x x} \ln F(x, y, t)+2 \sigma \rho \partial_{y y} \ln F(x, y, t)
$$

This says that we have a complex sea surface as a "source" and we want to find the function $F$ that solves the above Poisson equation. The constant $C$ removes the mean from the squared modulus 
$v|\Xi(x, y, t)|^{2}$. Osborne [1] gives methods for determining $|\psi(x, y, t)|^{2}=|\Xi(x, y, t)|^{2}$ from time series of the surface wave elevation $\eta(x, y, t)$ and its Hilbert transform $\widetilde{\eta}(x, y, t)$ (see Chapter 13). For water waves, $\sigma=-1$ and the operator in (75) is hyperbolic: Standard methods may be used for numerically solving the consequent hyperbolic Poisson equation given $|\psi(x, y, t)|^{2}(75)$.

At this juncture, we must keep in mind the relationships between $F$ and $G(x, y, t)=$ $a_{0} H\left(x, y, t \mid \boldsymbol{\tau}, \boldsymbol{\phi}^{-}\right) e^{i K^{\prime} x-i \Omega^{\prime} t}$ (the Hirota method) and the approximate asymptotic relation to theta functions (algebraic geometry) in anticipation of the leap later in the paper to the numerical methods of nonlinear Fourier analysis (determination of quasiperiodic Fourier series). To this end, we compute a function $H$ from knowledge of the complex surface elevation $\Xi(x, y, t)$ and $F(x, y, t)$ (plus modulation parameters $a_{0}, k_{0}, \omega_{0}$ ) by writing:

$$
\Xi(x, y, t)=a_{0} \frac{H(x, y, t)}{F(x, y, t)} e^{i k_{0} x-i\left(\omega_{0}+\Omega^{\prime}\right) t}
$$

We have assumed that $F$ and $H$ are related to the theta functions:

$$
\begin{aligned}
& F(x, y, t) \simeq \theta\left(x, y, t \mid \boldsymbol{\tau}, \boldsymbol{\phi}^{+}\right), \\
& H(x, y, t) \simeq \theta\left(x, y, t \mid \boldsymbol{\tau}, \boldsymbol{\phi}^{-}\right)
\end{aligned}
$$

Thus, we need to invert the two quasiperiodic Fourier series for (77) to get the parameters in the theta functions for the Riemann spectrum $\left\{\tau, \phi^{ \pm}\right\}$. The numerical approach for carrying out this process includes the numerical inversion of (76) and is given in Section 7.

\subsection{Computation of the Nonlinear Spectrum in Terms of the Riemann Matrix}

We need an estimate of the Riemann matrix and phases for the $2+1$ NLS equation for a given space or time series or wave field. Basically, the nonlinear directional spectrum of $2+1$ NLS consists of two contributions: (1) Each direction in the spectrum, characterized by an angle $\alpha$, corresponds to a "rotated 1+1 NLS equation." Thus, each direction has a one-dimensional NLS spectral description. (2) The nonlinear interactions among the rotated 1+1 NLS equations must also be included. Now the one-dimensional NLS equations, corresponding to each direction in the spectrum, are well known and their nonlinear spectra are also well known. Thus, to find the interactions among these $1+1$ directional NLS equations is an important goal. Stated differently, the Riemann spectra of each of the directional NLS equations must be pasted together to get an estimate of solutions of the $2+1$ NLS equation. We shall use the technique recently developed for the KP equation to obtain the necessary results (Osborne [4]). First, let us revisit known results about the Riemann spectrum for the $1+1$ NLS equation.

\subsection{Riemann Spectrum for the 1+1 NLS Equation}

Osborne [1] gives the Riemann spectrum for two phase-locked, coupled modes (a single breather) in the following form:

Small Expansion Parameter $\varepsilon_{i}$ and Sign of Riemann Sheet Index $\sigma_{i}$

$$
\begin{array}{lr}
\varepsilon_{1}=\varepsilon_{0} e^{i \theta} & \varepsilon_{2}=\varepsilon_{1}^{*} \\
\sigma_{1}=1 & \sigma_{2}=-1
\end{array}
$$

Spectral Eigenvalue

$$
\lambda_{1}=\lambda_{R}+i \lambda_{I} \quad \lambda_{2}=\lambda_{1}^{*}
$$

Spectral Wavenumber

$$
K_{1}=-2 \sqrt{A^{2}+\lambda_{1}^{2}} \quad K_{2}=-2 \sqrt{A^{2}+\lambda_{2}^{2}}
$$

Spectral Frequency

$$
\Omega_{1}=2 \lambda_{1} K_{1} \quad \Omega_{2}=2 \lambda_{2} K_{2}
$$


Period Matrix

$$
\begin{gathered}
\tau_{11}=\frac{1}{2}+\frac{i}{\pi} \ln \left(\frac{K_{1}^{2}}{\varepsilon_{1}}\right) \quad \tau_{12}=\frac{i}{2 \pi} \ln \left(\frac{1+\lambda_{1} \lambda_{2}+\frac{1}{4} K_{1} K_{2}}{1+\lambda_{1} \lambda_{2}-\frac{1}{4} K_{1} K_{2}}\right) \\
\tau_{21}=\tau_{12} \quad \tau_{22}=\frac{1}{2}+\frac{i}{\pi} \ln \left(\frac{K_{2}^{2}}{\varepsilon_{2}}\right)
\end{gathered}
$$

Phases

$$
\begin{aligned}
& \delta_{1}^{+}=\pi+i \ln \left(\lambda_{1}-\frac{1}{2} K_{1}\right)+i \ln \left(\sigma_{1} \lambda_{1}+\frac{1}{2} K_{1}\right) \\
& \delta_{1}^{-}=\pi+i \ln \left(\lambda_{1}+\frac{1}{2} K_{1}\right)+i \ln \left(\sigma_{1} \lambda_{1}+\frac{1}{2} K_{1}\right) \\
& \delta_{2}^{+}=\pi+i \ln \left(\lambda_{2}-\frac{1}{2} K_{2}\right)+i \ln \left(\sigma_{2} \lambda_{2}-\frac{1}{2} K_{2}\right) \\
& \delta_{2}^{-}=\pi+i \ln \left(\lambda_{2}+\frac{1}{2} K_{2}\right)+i \ln \left(\sigma_{2} \lambda_{2}-\frac{1}{2} K_{2}\right)
\end{aligned}
$$

The small expansion parameter $\varepsilon$ (78) of this method can be viewed as the "spine" connecting two points of simple spectrum (Osborne [1]). Use of the above formulas in the asymptotic theta function solution of the 2+1 NLS solution (69) provides a simple way to compute the unstable modes and breathers for a modulated plane wave carrier. However, because of the generality of the theta function formulation, these solutions describe not only the classical small-amplitude modulations (the Akhmediev breather, say), but also the large amplitude modulations (for example the Kuznetsov-Ma breather) as well (Osborne [1]).

\subsection{Riemann Spectrum for the 2+1 NLS Equation}

The "nearby integrability" that is the focus of this paper relates to two simple facts about the directional spectrum of $2+1$ NLS: (1) The spectrum consists of the individual spectra of the "rotated" $1+1$ NLS equations corresponding to each radial direction and (2) each rotated $1+1$ NLS equation nonlinearly interacts in a pairwise fashion with all the others. Each radial spectrum is described by a Riemann spectrum of the type shown in Equations (78)-(84). The Riemann spectrum describes Stokes waves on the diagonal elements of the Riemann matrix and interactions amongst the Stokes waves in the off-diagonal elements.

Therefore, the radial part is well known, but what about the interactions between the rotated $1+1$ NLS spectra? To this end, we use an argument relating to a similar study of the KP equation (Osborne [4]). In this study, we show that the solution of the KP equation can be found simply by rotating the solution of the $\mathrm{KdV}$ equation and the rotated dispersion relation and the resultant Riemann spectra are exactly those for the known solution of KP. In that derivation, we simply use the rotated off-diagonal terms of the KdV equation. In light of these results, we now use the rotated off-diagonal terms Equation (83) of the 1+1 NLS equation to get those for a "nearby" integrable case that is "asymptotic" to the $2+1$ NLS equation. In this way, there are only two further steps, which for lack of space we defer to a later paper, to complete the NLFA model of this paper: (1) put physical scaling in the Riemann spectrum Equations (78)-(84) (see Section 6 for a discussion on how to do this) and (2) use the interactions of the rotated Equation (83) to complete coupling of the rotated $1+1$ NLS spectra. The basic way to construct the period matrix for $2+1$ NLS is shown in Figure 17 below (placed there for later use), which essentially describes the schematic of the NLFA method used herein.

\section{The Physics of the NLFA Formulation}

\subsection{Overview of the Formulation}

The goal of this section is to simplify and amplify the physics of NLFA. We begin with Equation (75), which says that we have a spectral decomposition of the squared modulus in terms of the Riemann theta function with positive phase. First, recall that our ansatz solution of the $2+1$ NLS equation is written:

$$
\psi(x, y, t) \simeq a_{o} \frac{\theta\left(x, y, t \mid \boldsymbol{\tau}, \boldsymbol{\phi}^{-}\right)}{\theta\left(x, y, t \mid \tau, \boldsymbol{\phi}^{+}\right)} e^{i K^{\prime} x-i \Omega^{\prime} t}
$$


where

$$
\theta\left(x, y, t \mid \boldsymbol{\tau}, \boldsymbol{\phi}^{ \pm}\right)=\sum_{\mathbf{n} \in \mathbb{Z}^{N}} \theta_{\mathbf{n}} e^{i \mathbf{n} \cdot \mathbf{K}_{x} x+i \mathbf{n} \cdot \mathbf{K}_{y} y-i \mathbf{n} \cdot \mathbf{\Omega} t+i \mathbf{n} \cdot \boldsymbol{\phi}^{ \pm}}, \quad \theta_{\mathbf{n}}=e^{i \mathbf{n} \cdot \boldsymbol{\tau} \mathbf{n}}
$$

and the complex surface elevation is

$$
\Xi(x, y, t)=\eta(x, y, t)+\widetilde{\eta}(x, y, t) \simeq a_{0} \frac{\theta\left(x, y, t \mid \boldsymbol{\tau}, \boldsymbol{\phi}^{-}\right)}{\theta\left(x, y, t \mid \boldsymbol{\tau}, \boldsymbol{\phi}^{+}\right)} e^{i k_{0} x-i\left(\omega_{0}+\Omega^{\prime}\right) t}
$$

where $\eta(x, y, t)$ is the real, actual surface wave elevation and $\widetilde{\eta}(x, y, t)$ is its Hilbert transform. The simplest way to understand the actual modulation is to address the formulation of (87) as a modulus and phase:

$$
\Xi(x, y, t)=|\Xi(x, y, t)| e^{i \Phi(x, y, t)+i\left(k_{0}+K^{\prime}\right) x-i\left(\omega_{0}+\Omega^{\prime}\right) t}
$$

The goal of this section is to understand the modulus of a measured wave train $|\Xi(x, y, t)|$ and its nonlinear spectral decomposition. Physically, $|\Xi(x, y, t)|$ is what you see with the naked eye for the modulation. Look at a rapidly oscillating measured time series and then connect a smooth line through the maxima: This smooth line is the modulus of the instability, which we call $|\Xi(x, y, t)|=$ $\left[\eta^{2}(x, y, t)+\widetilde{\eta}^{2}(x, y, t)\right]^{1 / 2}$. It is clear that twice $|\Xi(x, y, t)|$ is the space/time wave height field $\mathrm{H}(x, y, t)=$ $2|\Xi(x, y, t)|$ (strictly speaking for a narrow banded spectrum, see Longuet-Higgins [41]). In Section 9 below we give an actual example of the modulus of stochastic breather trains from Currituck Sound [5] where $|\Xi(x, y, t)|$ is graphed together with the measured time series. When a breather packet propagates dynamically and surges (or breathes) to its maximum amplitude, one sees a substantial peak in the function $|\Xi(x, y, t)|$. One then seeks how to understand the nonlinear Fourier representation for $|\Xi(x, y, t)|$ or the wave height $\mathrm{H}(x, y, t)$, which is the most important problem we must solve in NLFA, see discussion in Section 7 on numerical methods. Indeed, understanding the dynamical and statistical behavior of the wave height $\mathrm{H}(x, y, t)$ are among the most important environmental inputs for offshore structure or ship design. Once we know $|\Xi(x, y, t)|$, we can determine the phase from (88):

$$
\Phi(x, y, t)=-i \ln \left[\frac{\Xi(x, y, t)}{|\Xi(x, y, t)|}\right]-\left(k_{o}+K^{\prime}\right) x+\left(\omega_{o}+\Omega^{\prime}\right) t
$$

By removing the linear trend in $x$ and $y$, we find $k_{o}+K^{\prime}$ and $\omega_{o}+\Omega^{\prime}$. Since $k_{o}$ and $\omega_{o}$ are known from the Fourier transform of the measured data field, we have estimates of the nonlinear Stokes corrections $K^{\prime}, \Omega^{\prime}$. This is the most general way to determine the nonlinear Stokes wavenumber and frequency corrections for a random wave field. For the one-dimensional problem, periodic inverse scattering gives the exact results in terms of algebraic geometric loop integrals (Tracy and Chen [21]).

\subsection{A Simple Example for a Single Degree of Freedom}

We begin with Equation (75), which we write in dimensional form:

$$
|\Xi(x, y, t)|^{2}-\left\langle|\Xi(x, y, t)|^{2}\right\rangle=\frac{2 \mu}{v}\left[\partial_{x x} \ln \theta\left(x, y, t \mid \boldsymbol{\tau}, \boldsymbol{\phi}^{+}\right)-2 \partial_{y y} \ln \theta\left(x, y, t \mid \boldsymbol{\tau}, \boldsymbol{\phi}^{+}\right)\right]
$$

where we have used $F(x, y, t) \simeq \theta\left(x, y, t \mid \tau, \boldsymbol{\phi}^{+}\right)$. The goal is to use Equation (86) in Equation (89) to theoretically determine the squared modulus. In general, this is a hard problem (numerically it is explained in Section 7), but for illustrative purposes it is interesting to consider the simplest form for Equation (86). We consider the case for: (1) A single Stokes mode, (2) truncated to first order in the nome $q$ for simplicity and (3) reduced to one-dimensional $x$. This results in

$$
\theta\left(x, t \mid \tau, \boldsymbol{\phi}^{+}\right) \simeq 1+2 q \cos (K x-\Omega t)
$$


where

$$
q=e^{i \pi \tau}
$$

and $\tau$ is a complex number, the $1 \times 1$ period matrix. Here, one assumes small nonlinearity, so that $|q|<<1$. The arbitrary phase is set to zero.

Let us see how this is carried out in a simple example. First, compute (89) with (90):

$$
|\psi(x, t)|^{2}-\left\langle|\psi|^{2}\right\rangle=\frac{2 \mu}{v} \partial_{x x} \ln F \simeq-\frac{4 \mu K^{2} q}{v} \frac{[2 q+\cos (K x-\Omega t)]}{[1+2 q \cos (K x-\Omega t)]^{2}}, \quad \rho=\sqrt{\frac{v}{2 \mu}}=\sqrt{2} k_{o}^{2}
$$

where the average is given by

$$
\left\langle|\psi|^{2}\right\rangle=\frac{2 \mu}{v} C=\frac{1}{2 \pi} \int_{0}^{2 \pi}|\psi(x)|^{2} d x=\frac{2}{\sqrt{1-4 q^{2}}}-1
$$

It is more convenient to write

$$
|\psi(x, t)|^{2}=\frac{2 \mu}{v} C+\frac{2 \mu}{v} \partial_{x x} \ln \theta(x, t)=\frac{2 \mu}{v} C\left[1+\frac{1}{C} \partial_{x x} \ln \theta(x, t)\right]=a_{o}^{2}\left[1+\frac{1}{C} \partial_{x x} \ln \theta(x, t)\right]
$$

where we have set the squared carrier amplitude $a_{0}^{2}$ to

$$
a_{o}^{2}=\frac{2 \mu}{v} C=\frac{1}{\rho^{2}} C
$$

so that

$$
C=\rho^{2} a_{o}^{2}
$$

Thus, we have the carrier amplitude

$$
a_{o}=\frac{1}{\rho}\left(\frac{2}{\sqrt{1-4 q^{2}}}-1\right)^{1 / 2}
$$

We see that, as the nonlinearity parameter $q$ becomes very small, then $a_{0}=1 / \rho$, a constant independent of nonlinearity.

Now we take the square root of (94) to get

$$
|\psi(x, t)| \simeq a_{o}\left[1+\varepsilon_{o} \partial_{x x} \ln \theta(x, t)\right], \quad \varepsilon_{o}=\frac{1}{2 C}=\frac{1}{2 \rho^{2} a_{o}^{2}}
$$

Then, use (90) in (98) to get

$$
|\psi(x, t)| \simeq a_{o}\left[1-\varepsilon \frac{2 q+\cos (K x-\Omega t)}{[1+2 q \cos (K x-\Omega t)]^{2}}\right], \quad \varepsilon=2 \varepsilon_{0} K^{2} q=\frac{K^{2} q}{\rho^{2} a_{o}^{2}}
$$

One should recognize the small parameter $\varepsilon$ in (99) is the loop integral expansion parameter in Equation (78) for the Riemann theta function: For near homoclinic motion, $\varepsilon$ covers the spine spectrum in the lambda plane (Osborne [1]). At this point, it is convenient to derive from the second formula of (99) the parameter $q$

$$
q=\left(\frac{\rho a_{o}}{K}\right)^{2} \varepsilon=\frac{1}{4}\left(\frac{2 \rho a_{0}}{K}\right)^{2} \varepsilon=\frac{1}{4} I_{B F}^{2} \varepsilon, \quad I_{B F}=\frac{2 \rho a_{0}}{K}
$$


$I_{B F}$ is the BF modulation parameter mentioned earlier. Then, in deep water, $\rho=\sqrt{2} k_{o}^{2}$ so that

$$
q=\frac{1}{4}\left(\frac{2 \sqrt{2} k_{o}^{2} a_{o}}{K}\right)^{2} \varepsilon=\frac{1}{4} I_{B F}^{2} \varepsilon, \quad I_{B F}=\frac{2 \sqrt{2} k_{o}^{2} a_{o}}{K}
$$

Now, from (91), we find the period matrix element as a function of the general parameters of the $1+1$ NLS equation, which is a function of arbitrary water depth $h$ :

$$
\tau=-\frac{i}{\pi} \ln q=\frac{i}{\pi} \ln q^{-1}=-\frac{i}{\pi} \ln \frac{1}{4}\left(\frac{2 \rho a_{0}}{K}\right)^{2} \varepsilon=-\frac{i}{\pi} \ln \frac{1}{4} I_{B F}^{2} \varepsilon=\frac{i}{\pi} \ln \left(\frac{K^{2}}{\rho^{2} a_{o}^{2} \varepsilon}\right) \varepsilon, \quad \rho=\sqrt{\frac{\nu}{2 \mu}}
$$

In addition, in the deep water limit, the period matrix element is:

$$
\tau=-\frac{i}{\pi} \ln q=\frac{i}{\pi} \ln q^{-1}=-\frac{i}{\pi} \ln \frac{1}{4}\left(\frac{2 \sqrt{2} k_{o}^{2} a_{o}}{K}\right)^{2} \varepsilon=-\frac{i}{\pi} \ln \frac{1}{4} I_{B F}^{2} \varepsilon=\frac{i}{\pi} \ln \left(\frac{K^{2}}{2 k_{o}^{4} a_{o}^{2} \varepsilon}\right) \varepsilon
$$

We see that the period matrix elements derived in (102) (103) are in full dimensional form. From (82), compared to (102), we see the necessary change of variables to get physical units in terms of the Benjamin-Feir parameter:

$$
K \rightarrow \frac{K}{\rho a_{o}}=\frac{K}{\sqrt{2} k_{o}^{2} a_{o}}
$$

The important thing in (102) and (103) is that we have found the diagonal elements of the period matrix are related to the Benjamin-Feir parameter of modulation theory. This is amazing because we have found a general result without using algebraic geometric loop integrals as found in Osborne [1].

We have found the physical expression for the period matrix $\tau$ is in terms of the BF parameter $I_{B F}$ and the small parameter $\varepsilon$ : This is the Riemann spectrum for a single Stokes wave.

\section{Numerical Methods of Nonlinear Fourier Analysis}

\subsection{Overview of Numerical Methods for Determining the Nonlinear Spectrum in $2+1$ Dimensions}

The goal is to pick the potential $U(x, y, t)$ to correspond to either the $2+1$ NLS equation, the Dysthe equation, or the Trulsen-Dysthe equations and to compute $F(x, y, t)$ by inverting (75) (see also Chapters $14-16$ and 23 in Osborne [1]). Then, given $F(x, y, t)$, we solve (72) or (74) to get $G(x, y, t)$. Of course, (74) is just (72) in Hirota operator notation, but there is some advantage in using the Hirota operator form. Finally, we have asymptotic solutions of $2+1$ NLS given by Equation (70).

Experimentally, an alternative approach is to solve (76) for $H(x, y, t)$ to get a fundamental relation

$$
H(x, y, t)=\Xi(x, y, t) F(x, y, t) \frac{e^{-i k_{0} x+i\left(\omega_{0}+\Omega^{\prime}\right) t}}{a_{0}}
$$

This result gives us the second theta function in Equation (77). Details about how to extract this part of the Riemann spectrum from data are given in the next subsection. By taking the amplitude and phase of the measured surface elevation, one can fit the Stokes frequency correction $\Omega^{\prime}$ directly from the data. These procedures are described in detail in the paper on the discovery of rogue waves in Currituck Sound (Osborne, et al. [5]).

\subsection{Procedure for Computing the NLFA Spectrum of the Measured Surface Wave Elevation $\eta(x, y, t)$}

Let us assume that, to maximize the possibilities for data analysis, we measure the surface wave amplitude for each value of time $t$, over a spatial grid in $x, y$ at intervals of $\Delta x, \Delta y$. There are $N_{x}, N_{y}$ spatial grid points. We continue to obtain these grid measurements at time intervals of $\Delta t$ for a total 
number of time points $N_{t}$. One might think of using lidar, radar, or synthetic aperture radar to make measurements of this type. Alternatively, primarily for organizational purposes, we could think of placing probes (buoys, resistance gauges, wave staffs, etc.) at each grid point: We would then be making time series measurements at each grid point. Both of these ways to make the measurements results in measuring the wave amplitude at each grid point for many values of time: We have a rectangular array of $N_{x} \times N_{y}$ spatial grid points and at each grid point we measure time series of $N_{t}$ points. What possibilities do we have to analyze these measurements to determine the Riemann spectrum $\{\tau, \omega, \boldsymbol{\phi}$, i.e., the Riemann matrix $\tau$, the frequency vector $\boldsymbol{\omega}$, and the phases $\phi$ ? The spatial measurements will have constant grid intervals $\Delta x, \Delta y$ and commensurable wavenumbers just as a $2 \mathrm{D}$ discrete Fourier transform or FFT. The time series, on the other hand, have constant $\Delta t$, but incommensurable frequencies. Computation of these frequencies using the nonlinear dispersion relation and knowledge of the modulational instability at increasing orders is discussed in a later subsection. Here is a list of numerical operations we must perform to compute the nonlinear spectrum:

(1) The first step is to compute the complex surface elevation $\Xi(x, y, t)=\eta(x, y, t)+\widetilde{\eta}(x, y, t)$ from a self-consistent definition of the 2D Hilbert transform, $\widetilde{\eta}(x, y, t)=H[\eta(x, y, t)]$. The paper by Zakharov and Kuznetsov [33] provides a mathematical/physical definition of the Hilbert transform in two dimensions. They give an asymptotic derivation of the Davey-Stewartson equations (which describes a 2D complex envelope $\psi(x, y, t)$ ) from the KP equation (which describes a 2D surface wave elevation $\eta(x, y, t))$ : In this way, one leaps from a 2D surface wave elevation $\eta(x, y, t)$ to a 2D complex envelope $\psi(x, y, t)$ by the formal methods of asymptotics. Numerically, the $2 \mathrm{D}$ Hilbert transform algorithm is well known.

(2) The second step is to compute the solution $F(x, y, t)$ of the hyperbolic Poisson equation:

$$
\mu \partial_{x x} \ln F(x, y, t)+\sigma \rho \partial_{y y} \ln F(x, y, t)=\frac{1}{2} U(x, y, t)=v|\Xi(x, y, t)|^{2}=v\left|\eta^{2}(x, y, t)+\widetilde{\eta}^{2}(x, y, t)\right|
$$

This equation says that the squared amplitude of the surface wave elevation (a real function) is the "source term" of (105). The solution gives the wavy motion corresponding to the positive phase theta function $F(x, y, t)=\theta\left(x, y, t \mid \boldsymbol{\tau}, \boldsymbol{\phi}^{+}\right)$. This means that the squared modulus of the (real) envelope of the complex surface elevation uniquely contains both the period matrix $\tau$ and the positive phases $\boldsymbol{\phi}^{+}$. We have just shown that these parameters are computable from measured data: Thus, we seek below the Riemann spectrum $\tau, \boldsymbol{\phi}^{+}$from this measured space/time series.

If we go to higher order beyond the squared modulus (the Dysthe and Trulsen-Dysthe equations), then we need terms like

$$
\partial_{x}|\psi|^{2}=\partial_{x}\left(\psi \psi^{*}\right)=\psi_{x} \psi^{*}+\psi \psi_{x}^{*}=\partial_{x}|\Xi|^{2}=\partial_{x}\left(\Xi \Xi^{*}\right)=\partial_{x} \Xi \Xi^{*}=\Xi_{x} \Xi^{*}+\Xi^{*} \Xi_{x}^{*}
$$

and others, to get the higher order results. This step is important because it means that we can go to a higher order using the function $\Xi(x, y, t)$ that we already know, i.e., via the 2D Hilbert transform of the measured surface wave elevation.

(3) The third step is to remove the carrier amplitude $a_{0}$, carrier wavenumber $k_{0}$, and carrier frequency $\omega_{0}$ to get a time series of the Stokes frequency correction: This should be removable by noting the linear phase behavior of $\Omega^{\prime} t$ in the data, and this can be fit to a straight temporal line whose slope is the Stokes frequency correction $\Omega^{\prime}$. These steps are made clearer in the next step (4).

(4) The fourth step is to compute the following function:

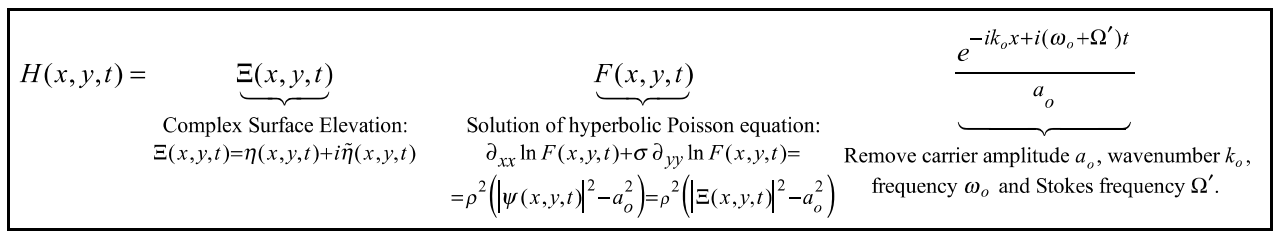


Equation (106) is the experimentally determined function related to the theta function with negative phases: $H(x, y, t) \simeq \theta\left(x, y, t \mid \boldsymbol{\tau}, \boldsymbol{\phi}^{-}\right)$. This latter relation is obtained from the measured data or complex surface wave elevation $\Xi(x, y, t)$ and we now need to extract the Riemann spectrum $\tau, \boldsymbol{\phi}^{-}$ with the negative phases. Comparison of the two Riemann matrices found in each of the steps (2) and (4) should be made to test for noise in the algorithm: One may have experimental errors or one may have to go to higher order to improve the comparison.

\subsection{Interpretation of Boxed Equation (106)}

(1) Begin with the complex surface wave elevation $\Xi(x, y, t)$, which is a rapidly oscillating function in both space $x, y$ and time $t$ and from this remove the carrier oscillation together with Stokes correction

$$
a_{0} e^{i k_{0} x-i\left(\omega_{0}+\Omega^{\prime}\right) t}
$$

to get a slowly oscillating modulation function. This shifts the Fourier transform from the carrier wavenumber and frequency to nearly zero, just as we physically expect. This is the well-known Fourier shifting theorem at work.

(2) Then, we multiply by the function $F(x, y, t)$, which oscillates over long spatial scales in $x, y$ and is slowly oscillating in $t$ because it is a measure of the squared modulus of the surface wave elevation via (106).

(3) We now have the function $H(x, y, t)$, that also has long spatial scales $x, y$ and slowly varying time $t$.

(4) Because $F(x, y, t)$ and $H(x, y, t)$ are long in $x, y$ and slow in time $t$, they are "theta function like" and therefore good for describing the envelope function $H(x, y, t) / F(x, y, t)$ where $H(x, y, t) \simeq$ $\theta\left(x, y, t \mid \boldsymbol{\tau}, \boldsymbol{\phi}^{-}\right)$and $F(x, y, t) \simeq \theta\left(x, y, t \mid \boldsymbol{\tau}, \boldsymbol{\phi}^{+}\right)$.

The Next Step: Determine the Riemann spectrum from the relations $H(x, y, t) \simeq \theta\left(x, y, t \mid \tau, \boldsymbol{\phi}^{-}\right)$and $F(x, y, t) \simeq \theta\left(x, y, t \mid \boldsymbol{\tau}, \boldsymbol{\phi}^{+}\right)$. Since $F(x, y, t)$ and $H(x, y, t)$ are determined from the data (they are effectively space and time series of the original data) and this means one should be able to get the Riemann spectrum $\left\{\boldsymbol{\tau}, \boldsymbol{\Phi}^{-}, \boldsymbol{\phi}^{+}\right\}$and that is what we want as the basic result, i.e., get the nonlinear spectrum from measurements. From the point of view of remotely sensed measurements, this means one should take lidar, radar or synthetic aperture radar data. Then, the methods given herein can be fully exploited. A schematic of this approach is given in Figure 14. 


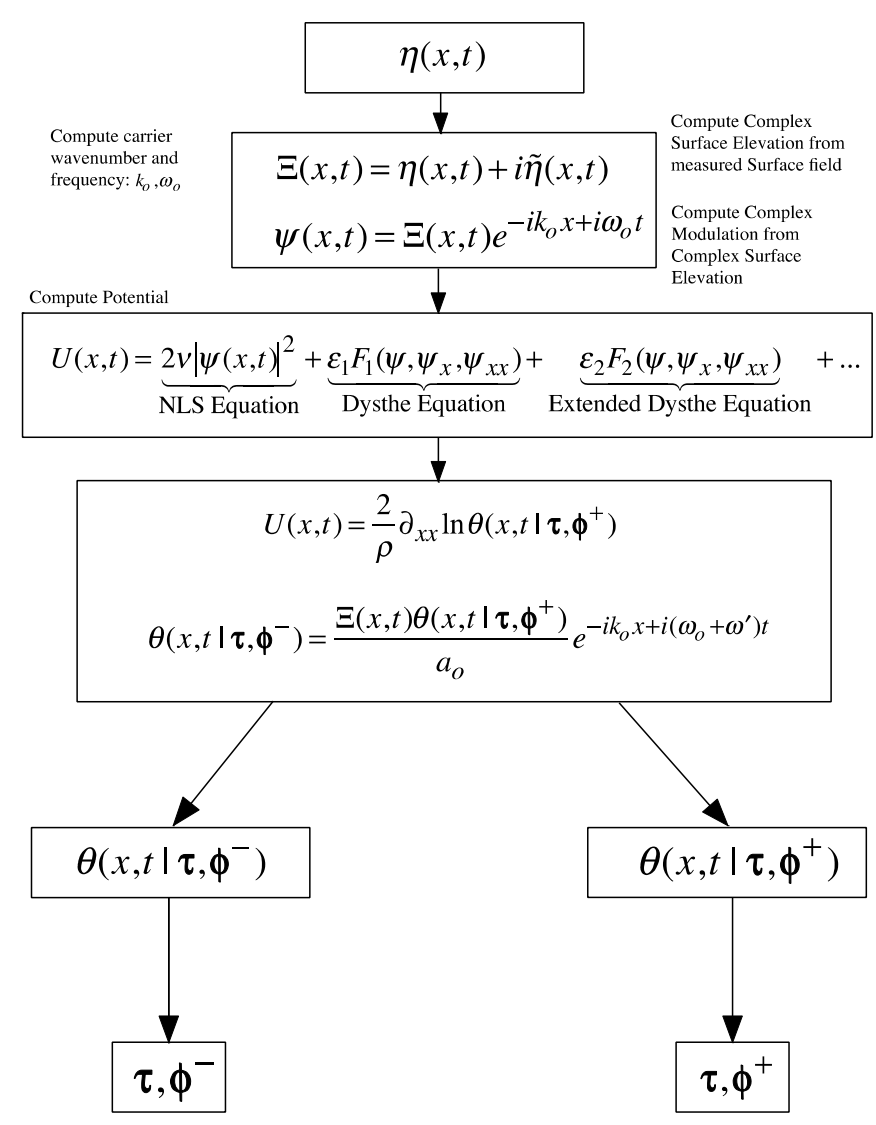

Figure 14. Determine the Riemann spectrum from measured data in one space and one-time dimensions.

\subsection{Quasiperiodic Fourier Structure of Measured Riemann Theta Functions}

We have discussed how to physically measure the space/time fields for $H(x, y, t) \simeq \theta\left(x, y, t \mid \boldsymbol{\tau}, \boldsymbol{\phi}^{-}\right)$ and $F(x, y, t) \simeq \theta\left(x, y, t \mid \tau, \boldsymbol{\phi}^{+}\right)$. Once we have these measurements we need to understand their quasiperiodic Fourier structure, which we now study.

The theta function for the positive and negative phases has the form

$$
\theta\left(x, y, t \mid \boldsymbol{\tau}, \boldsymbol{\Phi}^{\mp}\right)=\sum_{\mathbf{n} \in \mathbb{Z}} \theta_{\mathbf{n}} e^{i \mathbf{n} \cdot \boldsymbol{k} x+i \mathbf{n} \cdot \boldsymbol{\lambda}} y-i \mathbf{n} \cdot \mathbf{\Omega} t+i \mathbf{n} \cdot \boldsymbol{\phi}^{\mp}, \quad \theta_{\mathbf{n}}=e^{-i \pi \mathbf{n} \cdot \boldsymbol{\tau} \mathbf{n}}
$$

where we have taken $\boldsymbol{\kappa}=\mathbf{K}_{x}$ and $\lambda=\mathbf{K}_{y}$ in (86). Now, introduce spatially periodic boundary conditions (Osborne [1], Chapter 32) to obtain:

$$
\begin{aligned}
\theta\left(x, y, t \mid \boldsymbol{\tau}, \boldsymbol{\phi}^{ \pm}\right) & =\sum_{m=-\infty}^{\infty} \sum_{n=-\infty}^{\infty} \theta_{m n}^{ \pm}(t) e^{i k_{n} x+i l_{m} y}=F(x, y, t) \\
\theta_{m n}^{ \pm}(t) & =\sum_{\{\mathbf{n} \in \mathbb{Z}: m=I(\mathbf{n} \cdot \boldsymbol{\kappa}), n=I(\mathbf{n} \cdot \boldsymbol{\lambda})\}} \theta_{\mathbf{n}} e^{-i \mathbf{n} \cdot \boldsymbol{\Omega} t+i \mathbf{n} \cdot \boldsymbol{\phi}^{ \pm}}
\end{aligned}
$$

where we have $\theta\left(x, y, t \mid \boldsymbol{\tau}, \boldsymbol{\phi}^{ \pm}\right)=\theta\left(x+L_{x}, y, t \mid \boldsymbol{\tau}, \boldsymbol{\phi}^{ \pm}\right)=\theta\left(x, y+L_{y}, t \mid \boldsymbol{\tau}, \boldsymbol{\phi}^{ \pm}\right)$. If one is not familiar with this result, it would be best to first go to Chapter 32 of [1] to make sure that the notation is understood. The first equation of (108) says that the theta function can be represented as an ordinary 2D Fourier series with time varying coefficients. This is an important result because numerically we can, for spatially periodic boundary conditions, compute theta functions with the 2D FFT. The second equation gives the time varying coefficients as quasiperiodic Fourier series in time, which must be 
computed separately. We could equivalently write the second of (108) in a different notation using the Kronecker delta function:

$$
\theta_{m n}^{ \pm}(t)=\sum_{\mathbf{n} \in \mathbb{Z}} \theta_{\mathbf{n}}^{ \pm} e^{-i \mathbf{n} \cdot \mathbf{\Omega} t}, \quad \theta_{\mathbf{n}}^{ \pm}=\theta_{\mathbf{n}} \delta_{m, I(\mathbf{n} \cdot \mathbf{k})} \delta_{n, I(\mathbf{n} \cdot \boldsymbol{\lambda})} e^{i \mathbf{n} \cdot \boldsymbol{\phi}^{ \pm}}
$$

where

$$
\delta_{m, n}=\left\{\begin{array}{cc}
1, & m=n \\
0, & m \neq n
\end{array}\right\}
$$

This makes clear the notation in the second equation of (108). The inverse problem, which allows us to determine the Riemann spectrum $\left\{\tau, \boldsymbol{\phi}^{ \pm}\right\}$(the nonlinear spectral information about sine, Stokes and rogue waves) from the quasiperiodic Fourier series (107), (108), is given by the Fourier integral:

$$
\theta_{\mathbf{n}}^{ \pm}=\theta_{\mathbf{n}} \delta_{m, I(\mathbf{n} \cdot \mathbf{k})} \delta_{n, I(\mathbf{n} \cdot \boldsymbol{\lambda})} e^{i \mathbf{n} \cdot \boldsymbol{\phi}^{ \pm}}=\underbrace{\lim }_{T \rightarrow \infty} \frac{1}{T} \int_{0}^{T} \theta_{m n}^{ \pm}(t) e^{i \mathbf{n} \cdot \mathbf{\Omega} t} d t
$$

In summary, we first measure the surface elevation $\eta(x, y, t)$ by any of the known methods already discussed. We then compute the 2D Hilbert transform to obtain $\widetilde{\eta}(x, y, t)$. These are then used as inputs to Equation (105) from which we obtain $F(x, y, t)=\theta\left(x, y, t \mid \boldsymbol{\tau}, \boldsymbol{\phi}^{+}\right)$. From Equation (106), we obtain $H(x, y, t) \simeq \theta\left(x, y, t \mid \tau, \boldsymbol{\phi}^{-}\right)$. From Equation (108), we obtain the Fourier transform of the theta functions as $\theta_{m n}^{ \pm}(t)$. The $\theta_{m n}^{ \pm}(t)$ can be used to compute the Riemann spectrum $\left\{\boldsymbol{\tau}, \boldsymbol{\phi}^{ \pm}\right\}$from Equation (111) as seen in the expanded analysis below. Additional organizational material and an example problem, illustrating the determination of $\left\{\boldsymbol{\tau}, \boldsymbol{\phi}^{ \pm}\right\}$in one-space and one-time dimensions, are also given below.

One of the main advantages of the NLFA method presented here is that we are removing the necessity of doing algebraic geometry, but we use complex analysis instead.

We can write NLFA in terms of spatially periodic boundary conditions for two sets of phases $\boldsymbol{\phi}^{ \pm}$, the first of which is:

$$
\begin{aligned}
\theta\left(x, y, t \mid \boldsymbol{\tau}, \boldsymbol{\phi}^{-}\right) & =\sum_{m=-\infty}^{\infty} \sum_{n=-\infty}^{\infty} \theta_{m n}^{-}(t) e^{i k_{n} x+i l_{m} y}=H(x, y, t) \\
\theta_{m n}^{-}(t) & =\sum_{\{\mathbf{n} \in \mathbb{Z}: m=I(\mathbf{n} \cdot \mathbf{\kappa}), n=I(\mathbf{n} \cdot \boldsymbol{\lambda})\}} \theta_{\mathbf{n}} e^{-i \mathbf{n} \cdot \mathbf{\Omega} t+i \mathbf{n} \cdot \boldsymbol{\phi}^{-}}
\end{aligned}
$$

This means that, by having computed $H(x, y, t) \simeq \theta\left(x, y, t \mid \boldsymbol{\tau}, \boldsymbol{\phi}^{-}\right)$from the measured wave surface elevation $\eta(x, y, t)$, we can compute the spectrum $\left\{\tau, \boldsymbol{\phi}^{-}\right\}$. To obtain $F(x, y, t)$, we can compute the second set of phases:

$$
\begin{aligned}
\theta\left(x, y, t \mid \boldsymbol{\tau}, \boldsymbol{\phi}^{+}\right) & =\sum_{m=-\infty}^{\infty} \sum_{n=-\infty}^{\infty} \theta_{m n}^{+}(t) e^{i k_{n} x+i l_{m} y}=F(x, y, t) \\
\theta_{m n}^{+}(t) & =\sum_{\{\mathbf{n} \in \mathbb{Z}: m=I(\mathbf{n} \cdot \mathbf{k}), n=I(\mathbf{n} \cdot \boldsymbol{\lambda})\}} \theta_{\mathbf{n}} e^{-i \mathbf{n} \cdot \mathbf{\Omega} t+i \mathbf{n} \cdot \boldsymbol{\phi}^{+}}
\end{aligned}
$$

After computing $F(x, y, t)$ from the measured wave surface elevation $\eta(x, y, t)$, we can compute the spectrum $\left\{\tau, \boldsymbol{\phi}^{+}\right\}$. In this operation, we could easily take $t=0$, meaning that no information about the frequency could be obtained: Only information about the period matrix and the positive phases would arise in this case.

This means that we can take the 2D Fourier transform for each of the above measured functions $\theta\left(x, y, t \mid \boldsymbol{\tau}, \boldsymbol{\phi}^{+}\right), \theta\left(x, y, t \mid \boldsymbol{\tau}, \boldsymbol{\phi}^{-}\right)$to get $\theta_{m n}^{-}(t)$ and $\theta_{m n}^{+}(t)$. The inversion process exists because we have incommensurable frequencies (the second equations of (112) and (113)). We can therefore invert this series about the time variable; more on this is discussed below. 
7.5. Numerical Analysis in Two Dimensions: Measurement of a Wave Field of the Sea Surface at $t=0$

Now, suppose we have only spatial measurements of the wave field $\eta(x, y, 0)$ (radar or SAR measurements, say) at $t=0$. Then, it is convenient to determine the Fourier structure of the two phased theta functions at $t=0$ :

$$
\begin{gathered}
\theta\left(x, y, 0 \mid \boldsymbol{\tau}, \boldsymbol{\phi}^{-}\right)=\sum_{m=-\infty}^{\infty} \sum_{n=-\infty}^{\infty} \theta_{m n}^{-}(0) e^{i k_{n} x+i l_{m} y}=H(x, y, 0) \\
\theta_{m n}^{-}(0)=\sum_{\{\mathbf{n} \in \mathbb{Z}: m=I(\mathbf{n} \cdot \boldsymbol{\kappa}), n=I(\mathbf{n} \cdot \boldsymbol{\lambda})\}} \theta_{\mathbf{n}} e^{i \mathbf{n} \cdot \boldsymbol{\phi}^{-}}
\end{gathered}
$$

and

$$
\begin{gathered}
\theta\left(x, y, 0 \mid \boldsymbol{\tau}, \boldsymbol{\phi}^{+}\right)=\sum_{m=-\infty}^{\infty} \sum_{n=-\infty}^{\infty} \theta_{m n}^{+}(0) e^{i k_{n} x+i l_{m} y}=F(x, y, 0) \\
\theta_{m n}^{+}(0)=\sum_{\{\mathbf{n} \in \mathbb{Z}: m=I(\mathbf{n} \cdot \boldsymbol{\kappa}), n=I(\mathbf{n} \cdot \boldsymbol{\lambda})\}} \theta_{\mathbf{n}} e^{i \mathbf{n} \cdot \boldsymbol{\Phi}^{+}}
\end{gathered}
$$

Inverting the relations for the two-dimensional Fourier series

$$
\begin{aligned}
& \theta_{m n}^{-}(0)=\sum_{\{\mathbf{n} \in \mathbb{Z}: m=I(\mathbf{n} \cdot \mathbf{k}), n=I(\mathbf{n} \cdot \boldsymbol{\lambda})\}} \theta_{\mathbf{n}} e^{i \mathbf{n} \cdot \boldsymbol{\phi}^{-}}=\sum_{\mathbf{n} \in \mathbb{Z}} \theta_{\mathbf{n}}^{\prime} e^{i \mathbf{n} \cdot \boldsymbol{\phi}^{-}} \\
& \theta_{m n}^{+}(0)=\sum_{\{\mathbf{n} \in \mathbb{Z}: m=I(\mathbf{n} \cdot \boldsymbol{\kappa}), n=I(\mathbf{n} \cdot \boldsymbol{\lambda})\}} \theta_{\mathbf{n}} e^{i \mathbf{n} \cdot \boldsymbol{\phi}^{+}}=\sum_{\mathbf{n} \in \mathbb{Z}} \theta_{\mathbf{n}}^{\prime} e^{i \mathbf{n} \cdot \boldsymbol{\phi}^{+}}
\end{aligned}
$$

where

$$
\theta_{\mathbf{n}}^{\prime}=\theta_{\mathbf{n}} \delta_{m, I(\mathbf{n} \cdot \mathbf{k})} \delta_{n, I(\mathbf{n} \cdot \boldsymbol{\lambda})}
$$

gives the Riemann spectrum we need to understand nonlinearity in measured wave fields:

$$
\theta_{\mathbf{n}}=e^{-i \pi \mathbf{n} \cdot \boldsymbol{\tau} \mathbf{n}}, \boldsymbol{\phi}^{-}, \boldsymbol{\phi}^{+}
$$

One possible way to carry out this inverse problem is to use the method known as Nonlinear Adiabatic Annealing on a Riemann Surface (NLAARS), see Chapter 23 in Osborne [1]. Other approaches are suggested below.

\subsection{Numerical Analysis for a 1D Sea Surface Elevation}

Let us think in terms of a full lidar scan of the sea surface along a single transect as though the waves are in 1D. We carry out multiple scans of the same line on the sea surface over a range of times. Thus, we effectively measure $\eta(x, t)$ and seek the NLFA spectrum. These kinds of measurements are also often taken in a long wave flume, using probes at many locations along the direction of propagation.

In this case, we will, as discussed above in 2D, need now in 1D the results for the negative phases

$$
\begin{gathered}
\theta\left(x, t \mid \boldsymbol{\tau}, \boldsymbol{\phi}^{-}\right)=\sum_{n=-\infty}^{\infty} \theta_{n}^{-}(t) e^{i k_{n} x} \equiv H(x, t) \\
\theta_{n}^{-}(t)=\sum_{\{\mathbf{n} \in \mathbb{Z}: n=I(\mathbf{n} \cdot \mathbf{k})\}} \theta_{\mathbf{n}} e^{-i \mathbf{n} \cdot \mathbf{\Omega} t+i \mathbf{n} \cdot \boldsymbol{\phi}^{-}}
\end{gathered}
$$

and the positive phases

$$
\begin{gathered}
\theta\left(x, t \mid \boldsymbol{\tau}, \boldsymbol{\Phi}^{+}\right)=\sum_{n=-\infty}^{\infty} \theta_{n}^{+}(t) e^{i k_{n} x} \equiv F(x, t) \\
\theta_{n}^{+}(t)=\sum_{\{\mathbf{n} \in \mathbb{Z}:} \sum_{n=I(\mathbf{n} \cdot \mathbf{k})\}} \theta_{\mathbf{n}} e^{-i \mathbf{n} \cdot \mathbf{\Omega} t+i \mathbf{n} \cdot \boldsymbol{\phi}^{+}}
\end{gathered}
$$


Now, we can rewrite the negative phase result with Kronecker deltas:

$$
\begin{gathered}
\theta\left(x, t \mid \boldsymbol{\tau}, \boldsymbol{\phi}^{-}\right)=\sum_{n=-\infty}^{\infty} \theta_{n}^{-}(t) e^{i k_{n} x} \equiv H(x, t) \\
\theta_{n}^{-}(t)=\sum_{\mathbf{n} \in \mathbb{Z}} \theta_{\mathbf{n}}^{\prime} e^{-i \mathbf{n} \cdot \Omega t+i \mathbf{n} \cdot \boldsymbol{\phi}^{-}}=\sum_{\mathbf{n} \in \mathbb{Z}}\left(\theta_{\mathbf{n}}^{\prime} e^{i \mathbf{n} \cdot \boldsymbol{\phi}^{-}}\right) e^{-i \mathbf{n} \cdot \mathbf{\Omega} t}
\end{gathered}
$$

so that

$$
\theta_{n}^{-}(t)=\sum_{\mathbf{n} \in \mathbb{Z}} \theta_{\mathbf{n}}^{-} e^{-i \mathbf{n} \cdot \mathbf{\Omega} t}, \quad \theta_{\mathbf{n}}^{-}=\theta_{\mathbf{n}} \delta_{m, I(\mathbf{n} \cdot \boldsymbol{\kappa})} e^{i \mathbf{n} \cdot \boldsymbol{\phi}^{-}}
$$

Finally, for the negative phases:

$$
\begin{aligned}
\theta\left(x, t \mid \boldsymbol{\tau}, \boldsymbol{\phi}^{-}\right) & =\sum_{n=-\infty}^{\infty} \theta_{n}^{-}(t) e^{i k_{n} x}=H(x, t) \\
\theta_{n}^{-}(t) & \left.=\sum_{\mathbf{n} \in \mathbb{Z}} \theta_{\mathbf{n}}^{-} e^{-i \mathbf{n} \cdot \mathbf{\Omega} t}, \quad \theta_{\mathbf{n}}^{-}=\theta_{\mathbf{n}} \delta_{n, I(\mathbf{n} \cdot \mathbf{\kappa}}\right)^{i \mathbf{n} \cdot \boldsymbol{\phi}^{+}}, \quad \theta_{\mathbf{n}}=e^{-i \pi \mathbf{n} \cdot \boldsymbol{\tau} \mathbf{n}}
\end{aligned}
$$

The inverse problem for the quasiperiodic Fourier series is given by:

$$
\theta_{\mathbf{n}}^{-}=\theta_{\mathbf{n}} \delta_{n, I(\mathbf{n} \cdot \boldsymbol{\kappa})} e^{i \mathbf{n} \cdot \boldsymbol{\phi}^{-}}=\underbrace{\lim _{T \rightarrow \infty}}_{T \rightarrow \infty} \frac{1}{T} \int_{0}^{T} \theta_{n}^{-}(t) e^{i \mathbf{n} \cdot \Omega t} d t, \quad \theta_{\mathbf{n}}=e^{-i \pi \mathbf{n} \cdot \boldsymbol{\tau} \mathbf{n}}
$$

This is the fundamental relation for determining the period matrix $\boldsymbol{\tau}$ and the negative phases $\boldsymbol{\phi}^{-}$. For the positive phases, we have finally:

$$
\begin{gathered}
\theta\left(x, t \mid \boldsymbol{\tau}, \boldsymbol{\phi}^{+}\right)=\sum_{n=-\infty}^{\infty} \theta_{n}^{+}(t) e^{i k_{n} x}=F(x, t) \\
\theta_{n}^{+}(t)=\sum_{\mathbf{n} \in \mathbb{Z}} \theta_{\mathbf{n}}^{+} e^{-i \mathbf{n} \cdot \mathbf{\Omega} t}, \quad \theta_{\mathbf{n}}^{+}=\theta_{\mathbf{n}} \delta_{n, I(\mathbf{n} \cdot \mathbf{\kappa})} e^{i \mathbf{n} \cdot \boldsymbol{\phi}^{+}}, \quad \theta_{\mathbf{n}}=e^{-i \pi \mathbf{n} \cdot \boldsymbol{\tau} \mathbf{n}} \\
\theta_{\mathbf{n}}^{+}=\theta_{\mathbf{n}} \delta_{n, I(\mathbf{n} \cdot \mathbf{\kappa})} e^{i \mathbf{n} \cdot \boldsymbol{\phi}^{+}}=\underbrace{\lim }_{T \rightarrow \infty} \frac{1}{T} \int_{0}^{T} \theta_{n}^{+}(t) e^{i \mathbf{n} \cdot \mathbf{\Omega} t} d t, \quad \theta_{\mathbf{n}}=e^{-i \pi \mathbf{n} \cdot \boldsymbol{\tau} \mathbf{n}}
\end{gathered}
$$

This is the fundamental relation for determining the period matrix $\boldsymbol{\tau}$ and the positive phases $\boldsymbol{\phi}^{+}$.

Nota bene: These formulas work if we get the sea surface elevation $\eta(x, t)$ by a lidar swipe over space $x$ $(0 \leq x \leq L)$, and multiple values $N_{t}$ of time $t$. By taking the steps given above, we compute the two functions $H(x, t)$ and $F(x, t)$ so that we obtain the theta functions $\theta\left(x, t \mid \tau, \boldsymbol{\phi}^{ \pm}\right)$for the two phases. We take the Fourier transforms of these functions to give

$$
\begin{aligned}
\theta\left(x, t \mid \tau, \boldsymbol{\phi}^{ \pm}\right) & =\sum_{n=-\infty}^{\infty} \theta_{n}^{ \pm}(t) e^{i k_{n} x} \\
\theta_{n}^{ \pm}(t) & =\sum_{\mathbf{n} \in \mathbb{Z}} \theta_{\mathbf{n}}^{ \pm} e^{-i \mathbf{n} \cdot \Omega t}, \quad \theta_{\mathbf{n}}^{ \pm}=\theta_{\mathbf{n}} \delta_{n, I(\mathbf{n} \cdot \mathbf{\kappa}} e^{i \mathbf{n} \cdot \boldsymbol{\phi}^{+}}, \quad \theta_{\mathbf{n}}=e^{-i \pi \mathbf{n} \cdot \boldsymbol{\tau} \mathbf{n}}
\end{aligned}
$$

Inverting the quasiperiodic Fourier series, we have

$$
\theta_{\mathbf{n}}^{ \pm}=\theta_{\mathbf{n}} \delta_{n, I}(\mathbf{n} \cdot \mathbf{k}) e^{i \mathbf{n} \cdot \phi^{ \pm}}=\underbrace{\lim }_{T \rightarrow \infty} \frac{1}{T} \int_{0}^{T} \theta_{n}^{ \pm}(t) e^{i \mathbf{n} \cdot \mathbf{\Omega} t} d t, \quad \theta_{\mathbf{n}}=e^{-i \pi \mathbf{n} \cdot \boldsymbol{\tau} \mathbf{n}}
$$


We thus obtain the theta spectrum $\left\{\tau, \boldsymbol{\phi}^{ \pm}\right\}$.

\subsection{Concrete Example for a Single Degree of Freedom}

First, writing the fundamental spectrum, we seek:

$$
\theta_{\mathbf{n}}^{ \pm}=\delta_{n, I(\mathbf{n} \cdot \boldsymbol{\kappa})} e^{-i \pi \mathbf{n} \cdot \boldsymbol{\tau} \mathbf{n}+i \mathbf{n} \cdot \boldsymbol{\phi}^{ \pm}}=\underbrace{\lim }_{T \rightarrow \infty} \frac{1}{T} \int_{0}^{T} \theta_{n}^{ \pm}(t) e^{i \mathbf{n} \cdot \Omega} d t
$$

Set $\mathbf{n}=[1,0,0 \ldots 0]$ (this is the simplest possible case: it says look at the first component of the first Stokes wave in the spectrum) to get

$$
\delta_{n, I([1,0,0, \ldots] \cdot \boldsymbol{\kappa})} e^{-i \pi[1,0,0 \ldots 0] \cdot \boldsymbol{\tau}[1,0,0, \ldots 0]+i[1,0,0, \ldots] \cdot \boldsymbol{\phi}^{ \pm}}=\underbrace{\lim }_{T \rightarrow \infty} \frac{1}{T} \int_{0}^{T} \theta_{n}^{ \pm}(t) e^{i[1,0,0 \ldots 0] \cdot \Omega t} d t
$$

Then, $n=I([1,0,0 \ldots 0] \cdot \boldsymbol{\kappa})=1$ (recall Equation $(68)$ and that $\left.\boldsymbol{\delta}_{1,1}=1\right)$ so that

$$
e^{-\pi i \tau_{11}+i \phi_{1}^{ \pm}}=\underbrace{\lim }_{T \rightarrow \infty} \frac{1}{T} \int_{0}^{T} \theta_{1}^{ \pm}(t) e^{i \Omega_{1} t} d t
$$

We can also set $\mathbf{n}=[-1,0,0 \ldots 0]$ to get

$$
\delta_{n, I([-1,0,0, \ldots 0] \cdot \mathbf{\kappa})} e^{-i \pi[-1,0,0 \ldots 0] \cdot \boldsymbol{\tau}[-1,0,0 \ldots 0]+i[-1,0,0 \ldots 0] \cdot \boldsymbol{\Phi}^{ \pm}}=\underbrace{\lim }_{T \rightarrow \infty} \frac{1}{T} \theta_{n}^{ \pm} e^{i[-1,0,0 \ldots 0] \cdot \Omega} d t
$$

Then, $n=I([-1,0,0 \ldots 0] \cdot \mathbf{k})=-1\left(\operatorname{set} n=-1, \delta_{-1,-1}=1\right)$ so that

$$
e^{-\pi i \tau_{11}-i \phi_{1}^{ \pm}}=\underbrace{\lim }_{T \rightarrow \infty} \frac{1}{T} \int_{0}^{T} \theta_{-1}^{ \pm}(t) e^{-i \Omega_{1} t} d t
$$

Take the natural logarithm of Equations (132) and (134)

$$
\begin{aligned}
& -\pi i \tau_{11}+i \phi_{1}^{ \pm}=\ln (\underbrace{\lim \frac{1}{T}}_{T \rightarrow \infty} \int_{0}^{T} \theta_{1}^{ \pm}(t) e^{i \Omega_{1} t} d t) \\
& -\pi i \tau_{11}-i \phi_{1}^{ \pm}=\ln (\underbrace{\lim }_{T \rightarrow \infty} \frac{1}{T} \int_{0}^{T} \theta_{-1}^{ \pm}(t) e^{-i \Omega_{1} t} d t)
\end{aligned}
$$

Add the last two equations to get the period matrix element:

$$
\tau_{11}=\frac{i}{2 \pi}\left\{\ln \left([\underbrace{\lim }_{T \rightarrow \infty} \frac{1}{T} \int_{0}^{T} \theta_{1}^{ \pm}(t) e^{i \Omega_{1} t} d t)(\underbrace{\lim }_{T \rightarrow \infty} \frac{1}{T} \int_{0}^{T} \theta_{-1}^{ \pm}(t) e^{-i \Omega_{1} t} d t)\right]\right\}
$$


Subtract the above two equations to get the phase:

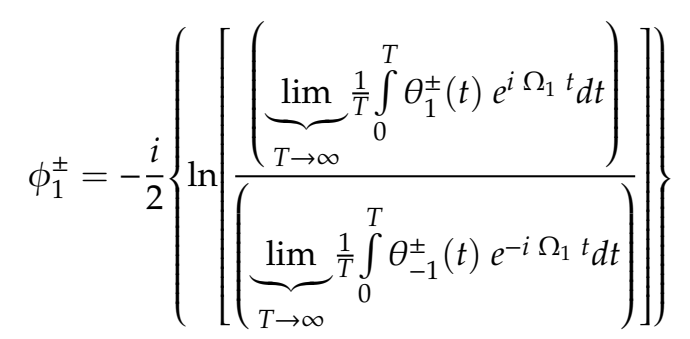

We have just found a way to numerically obtain the first diagonal element of the period matrix $\tau_{11}$ and the first phase components $\phi_{1}^{ \pm}$. We still need to get the remainder of the diagonal elements and the off diagonal elements. Notice that both the diagonal elements of the period matrix and the phases are generally complex numbers. This is an important departure from standard Fourier analysis.

To get the other diagonal elements, repeat the above computations but use the $j$ th component of the $\mathbf{n}$ vector

$$
\mathbf{n}=[0,0, \underbrace{ \pm 1}_{\substack{j \text { th } \\ \text { component }}}, 0 \ldots 0]
$$

where $j$ is the number of the component in the vector $\mathbf{n}: 0 \leq j \leq N$. This general procedure gives the $j$ th components of the diagonal elements of the period matrix $\tau_{j j}$ and the $j$ th phase $\phi_{j}$. Recall

$$
\theta_{\mathbf{n}}^{ \pm}=\delta_{n, I(\mathbf{n} \cdot \mathbf{k})} e^{-i \pi \mathbf{n} \cdot \boldsymbol{\tau} \mathbf{n}+i \mathbf{n} \cdot \boldsymbol{\phi}^{ \pm}}=\underbrace{\lim }_{T \rightarrow \infty} \frac{1}{T} \int_{0}^{T} \theta_{n}^{ \pm}(t) e^{i \mathbf{n} \cdot \Omega t} d t
$$

Use $\mathbf{n}=[0,0, \underbrace{+1}_{j \text { th }}, 0 \ldots 0]$ to get $(\operatorname{set} n=j)$

$$
\begin{gathered}
\delta_{n, j} e^{-i \pi \tau_{j j}+i \phi_{j}^{ \pm}}=e^{-\frac{1}{2} \tau_{j j}+i \phi_{j}^{ \pm}}=\underbrace{\lim }_{T \rightarrow \infty} \frac{1}{T} \int_{0}^{T} \theta_{j}^{ \pm}(t) e^{i \Omega_{j} t} d t \\
e^{-i \pi \tau_{j j}+i \phi_{j}^{ \pm}}=\underbrace{\lim }_{T \rightarrow \infty} \frac{1}{T} \int_{0}^{T} \theta_{j}^{ \pm}(t) e^{i \Omega_{j} t} d t
\end{gathered}
$$

Now use $\mathbf{n}=[0,0, \underbrace{-1}_{j \text { th }}, 0 \ldots 0]$ to get $(\operatorname{set} n=-j)$

$$
\begin{gathered}
\delta_{n,-j} e^{-i \pi \tau_{j j}-i \phi_{j}^{ \pm}}=e^{-i \pi \tau_{j j}-i \phi_{j}^{ \pm}}=\underbrace{\lim }_{T \rightarrow \infty} \frac{1}{T} \int_{0}^{T} \theta_{-j}^{ \pm}(t) e^{i \Omega_{j} t} d t \\
e^{-i \pi \tau_{j j}-i \phi_{j}^{ \pm}}=\underbrace{\lim }_{T \rightarrow \infty} \frac{1}{T} \int_{0}^{T} \theta_{-j}^{ \pm}(t) e^{i \Omega_{j} t} d t
\end{gathered}
$$


Add the logarithms of Equations (142) and (144) to get the period matrix element:

$$
\tau_{j j}=\frac{i}{2 \pi}\left\{\ln \left[(\underbrace{\lim }_{T \rightarrow \infty} \frac{1}{T} \int_{0}^{T} \theta_{j}^{ \pm}(t) e^{i \Omega_{j} t} d t)(\underbrace{\lim }_{T \rightarrow \infty} \frac{1}{T} \int_{0}^{T} \theta_{-j}^{ \pm}(t) e^{-i \Omega_{j} t} d t)\right]\right\}
$$

Subtract the logarithms of Equations (142) and (144) to get the phase:

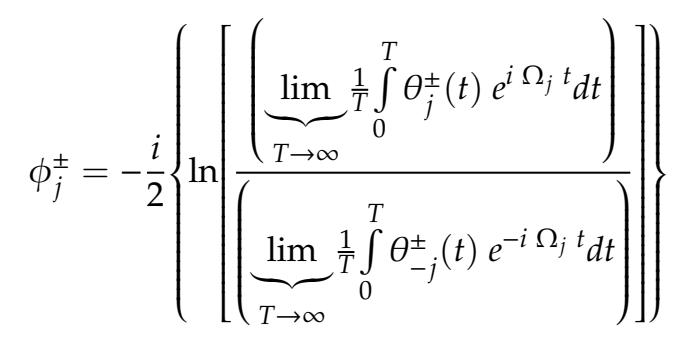

Likewise, to address the off diagonal elements $\tau_{j k}$, use $\mathbf{n}=[0,0, j, 0, k, 0 \ldots 0]$ where $i, k$ are the numbers of the components in the vector $\mathbf{n}: 0 \leq j, k \leq N$. This gives us the off-diagonal elements of the period matrix $\tau_{j k}$. We now generalize this procedure to also get the off-diagonal elements.

\subsection{Alternative General Notation}

We now see formally how to extract the Riemann matrix and phases from remotely sensed ocean wave data. This allows us to determine the wave motion in future times. Suppose we measure the surface elevation $\eta\left(x, t_{n}\right)$ by lidar swipes over $x$ for many values of time $t_{n}$ for $n=1,2 \ldots M$. For example, we might get a lidar swipe every one second for a $10 \mathrm{~min}$ interval, so that we get 600 swipes in this time period. We take the Fourier transform of each swipe and compute the Fourier coefficients for each value of time $\theta_{n}^{ \pm}(t)$. What follows is how to get the Riemann spectrum and phases from these time varying Fourier coefficients in a simpler but more general notation.

The notation is easier than that given in the last section. We begin with the inverse quasiperiodic Fourier series for the negative phases:

$$
\theta_{n}^{-}(t)=\sum_{\mathbf{n} \in \mathbb{Z}} \theta_{\mathbf{n}}^{-} e^{-i \mathbf{n} \cdot \Omega t}, \quad \theta_{\mathbf{n}}^{-}=\theta_{\mathbf{n}} \delta_{n, I(\mathbf{n} \cdot \mathbf{k})} e^{i \mathbf{n} \cdot \boldsymbol{\phi}^{-}}
$$

whose inverse is:

$$
\theta_{\mathbf{n}}^{-}=\theta_{\mathbf{n}} \delta_{n, I(\mathbf{n} \cdot \mathbf{k})} e^{i \mathbf{n} \cdot \boldsymbol{\phi}^{-}}=\underbrace{\lim _{T \rightarrow \infty}}_{T \rightarrow \infty} \frac{1}{T} \int_{0}^{T} \theta_{n}^{-}(t) e^{i \mathbf{n} \cdot \Omega t} d t
$$

which can also be written

$$
e^{-\pi i \mathbf{n} \cdot \boldsymbol{\tau} \mathbf{n}+i \mathbf{n} \cdot \boldsymbol{\phi}^{-}}=\underbrace{\lim }_{T \rightarrow \infty} \frac{1}{T} \int_{0}^{T} \theta_{n=I(\mathbf{n} \cdot \mathbf{\kappa})}^{-}(t) e^{i \mathbf{n} \cdot \mathbf{\Omega} t} d t
$$

Taking the logarithm, we have:

$$
-\pi i \mathbf{n} \cdot \mathbf{\tau n}+i \mathbf{n} \cdot \boldsymbol{\phi}^{-}=\ln (\underbrace{\lim }_{T \rightarrow \infty} \frac{1}{T} \int_{0}^{T} \theta_{n=I(\mathbf{n} \cdot \mathbf{k})}^{-}(t) e^{i \mathbf{n} \cdot \Omega t} d t)
$$


For the positive phases, we get a similar result

$$
-\pi i \mathbf{n} \cdot \mathbf{\tau} \mathbf{n}+\mathbf{n} \cdot \boldsymbol{\phi}^{+}=\ln (\underbrace{\lim \frac{1}{T}}_{T \rightarrow \infty} \int_{0}^{T} \theta_{n=I(\mathbf{n} \cdot \mathbf{\kappa})}^{+}(t) e^{i \mathbf{n} \cdot \mathbf{\Omega} t} d t)
$$

In summary, the general relation is

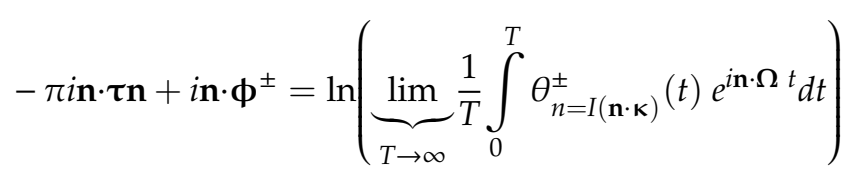

Now, we can apply the operation $\mathbf{n} \rightarrow-\mathbf{n}$, since we have "index $\mathbf{n}$ reversal symmetry" in the theta functions under the change in sign of the dummy summation index $\mathbf{n}$. The last relation Equation (152) becomes

$$
-\pi i \mathbf{n} \cdot \boldsymbol{\tau} \mathbf{n}-i \mathbf{n} \cdot \boldsymbol{\phi}^{ \pm}=\ln (\underbrace{\lim \frac{1}{T}}_{T \rightarrow \infty} \int_{0}^{T} \theta_{n=-I(\mathbf{n} \cdot \mathbf{k})}^{ \pm}(t) e^{-i \mathbf{n} \cdot \Omega t} d t)
$$

Now, add the last two equations to eliminate the phases:

$$
\mathbf{n} \cdot \mathbf{\tau n}=\frac{i}{2 \pi} \ln \left[(\underbrace{\lim }_{T \rightarrow \infty} \frac{1}{T} \int_{0}^{T} \theta_{n=I(\mathbf{n} \cdot \mathbf{k})}^{ \pm}(t) e^{i \mathbf{n} \cdot \Omega} t d t)(\underbrace{\lim }_{T \rightarrow \infty} \frac{1}{T} \int_{0}^{T} \theta_{n=-I(\mathbf{n} \cdot \mathbf{k})}^{ \pm}(t) e^{-i \mathbf{n} \cdot \Omega} t d t]\right.
$$

This gives a simple way to determine all the elements of the period matrix from the time varying Fourier coefficients of the input surface wave data.

Now, subtract Equations (152) and (153) to eliminate the period matrix:

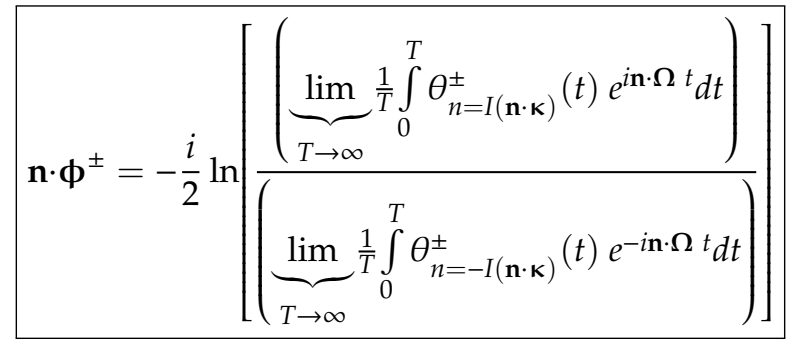

\subsection{Primer on Nonlinear Fourier Analysis as a Theory of Nonlinearly Interacting Stokes Waves}

The nonlinear Fourier theory we discuss in this paper has a spectrum that is a matrix, the so-called period or Riemann matrix: The diagonal elements $\tau_{i i}, i=1,2 \ldots N$ correspond to Stokes waves and the off-diagonal elements $\tau_{i j}, i=1,2 \ldots N, j=1,2 \ldots N$ correspond to pairwise interactions between the $i$ th and $j$ th Stokes waves in the spectrum. The $i$ th Stokes wave has harmonics that are phase locked with each other. In this way, each Stokes wave has a single phase $\phi_{i}$ and therefore is a "collection of bound modes" such that the $n$th harmonic has phase $n \phi_{i}$, so that the $N$ harmonics have phases $n \phi_{i} \Rightarrow \phi_{i}, 2 \phi_{i}, 3 \phi_{i}, \ldots N \phi_{i}$. Thus, each Stokes wave is a "free mode" that consists of a collection of "bound harmonics." In this way, we view the Stokes waves as "coherent structures" that maintain their form as they propagate and interact with each other. This perspective is always true in shallow water where the KdV and KP equations are valid. NLFA is just a theory of nonlinear directional spectra consisting of interacting Stokes waves in shallow water. One can go to higher Stokes order, but the picture remains essentially the same as long as one is interested in shallow water. 
In deep water, the situation is more interesting. Here, deep water just means that the modulational instability condition $k h>1.363$ holds $[1,40,48]$. These are the waves that are shorter than about 4.6 times the water depth (Osborne, Chapter 2, Figure 2) [1]. To see this, use $k=2 \pi / L$ in $k h>1.363$ and find $L<(2 \pi / 1.363) h=4.610 h$. In this case, NLFA borrows from periodic inverse scattering theory to produce the breather packets, provided that $I_{B F}>1$, which consist of two phase locked Stokes waves referred to as a breather (see Equations (78)-(84) and Osborne (Chapter 24) [1]). Each Stokes wave is a simple coherent structure and, when the condition $k h>1.363$ is met, the two Stokes waves couple with each other to form a "nonlinear beat" that we call a breather. The breathers form a reasonable mechanism for the generation of "rogue waves", i.e., for the extreme waves in the largest storms (Kharif et al. [59]). The present paper is primarily about the deep-water problem in one and two spatial dimensions.

We now give an overview of the NLFA computations made above for understanding deep-water waves. The two Equations (154) and (155) are the most general way to obtain the Riemann spectrum $\tau, \phi^{ \pm}$from measured data. One picks the index $\mathbf{n}$ to obtain the Fourier component that one wants. Let us see how to do this. Remember that the nonlinear spectrum consists of $N$ Stokes waves that have many components. This means in general that $\mathbf{n}=\left[n_{1}, n_{2}, n_{3}, n_{4} \ldots n_{N}\right]$ so that we have a total of $N$ Stokes waves in the spectrum, which formally are described by the diagonal elements of the period matrix. For example, $\mathbf{n}=[0,0,2,0 \ldots 0]$ means we want to select the 2 nd harmonic of the 3rd Stokes wave in the spectrum. Suppose that we want the $n$th component of the $j$ th Stokes wave. Then, we go to the $j$ th component of the vector $\mathbf{n}$ and set $n$ there:

$$
\mathbf{n}=[0,0, \underbrace{n}_{j}, 0 \ldots 0]
$$

Now, all the phases will be equal in a Stokes wave because all the components are phase locked. If we want to include the interactions among the Stokes waves, we would select, for example, the $i$ th and $j$ th Stokes wave and their $m$ th and $n$th components:

$$
\mathbf{n}=[0, \underbrace{m}_{i}, 0, \underbrace{n}_{j}, 0 \ldots 0]
$$

This involves the off-diagonal period matrix element $\tau_{i j}$, which describes the nonlinear interactions between the two Stokes waves from the $i$ th and $j$ th diagonal elements of the period matrix.

The above approach works for the two theta functions $\theta\left(x, y, t \mid \tau, \boldsymbol{\phi}^{ \pm}\right)$in the solution of $1+1$ NLS whose Fourier series we again write (recall this is for spatially periodic boundary conditions, just as we might assume with a lidar scan):

$$
\begin{aligned}
\theta\left(x, t \mid \boldsymbol{\tau}, \boldsymbol{\phi}^{ \pm}\right) & =\sum_{n=-\infty}^{\infty} \theta_{n}^{ \pm}(t) e^{i k_{n} x} \\
\theta_{n}^{ \pm}(t) & =\sum_{\mathbf{n} \in \mathbb{Z}} \theta_{\mathbf{n}}^{ \pm} e^{-i \mathbf{n} \cdot \Omega t}, \quad \theta_{\mathbf{n}}^{ \pm}=\delta_{n, I(\mathbf{n} \cdot \mathbf{\kappa})} e^{-i \pi \mathbf{n} \cdot \boldsymbol{\tau} \mathbf{n}+i \mathbf{n} \cdot \boldsymbol{\phi}^{ \pm}}
\end{aligned}
$$

Now, let

$$
\mathbf{n}=[0,0, \underbrace{1}_{j t h}, 0 \ldots 0]
$$


to get $\mathbf{n} \cdot \boldsymbol{\phi}^{ \pm}=\phi_{j}^{ \pm}\left(\right.$use $\left.\boldsymbol{\phi}^{ \pm}=\left[\phi_{1}^{ \pm}, \phi_{2}^{ \pm}, \phi_{3}^{ \pm} \ldots \phi_{N}^{ \pm}\right]\right), I(\mathbf{n} \cdot \boldsymbol{\kappa})=j\left(\right.$ use $\left.\kappa=\left[\kappa_{1}, \kappa_{2}, \kappa_{3} \ldots \kappa_{N}\right]\right)$ and $\mathbf{n} \cdot \boldsymbol{\tau} \mathbf{n}=\tau_{j j}$. This means we have the diagonal elements of the period matrix:

$$
\tau_{j j}=\frac{i}{2 \pi} \ln \left[(\underbrace{\lim }_{T \rightarrow \infty} \frac{1}{T} \int_{0}^{T} \theta_{n=j}^{ \pm}(t) e^{i \Omega_{j} t} d t)(\underbrace{\lim \frac{1}{T}}_{T \rightarrow \infty} \int_{0}^{T} \theta_{n=-j}^{ \pm}(t) e^{-i \Omega_{j} t} d t)\right]
$$

Likewise, we have the phases:

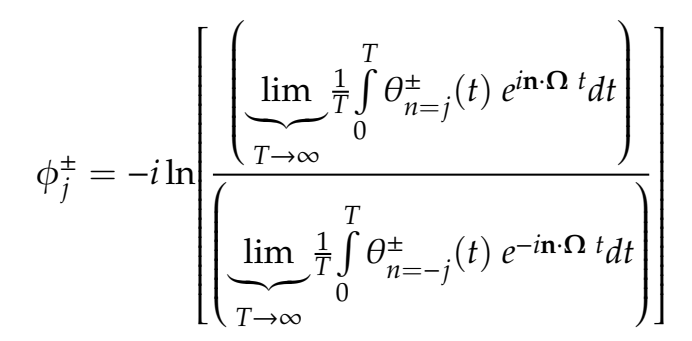

This is Equation (155) in a different notation. To get the off diagonal elements of the period matrix, let

$$
\mathbf{n}=[0,0, \underbrace{1}_{j t h}, 0, \underbrace{1}_{k t h}, 0 \ldots 0]
$$

Then,

$$
\begin{gathered}
\mathbf{n} \cdot \boldsymbol{\phi}^{ \pm}=\phi_{j}^{ \pm}+\phi_{k}^{ \pm} \\
I(\mathbf{n} \cdot \boldsymbol{\kappa})=j+k \\
\mathbf{n} \cdot \boldsymbol{\tau} \mathbf{n}=\tau_{j j}+2 \tau_{j k}+\tau_{k k}
\end{gathered}
$$

The last equation occurs because, as we see for two degrees of freedom:

$$
\mathbf{n} \cdot \boldsymbol{\tau} \mathbf{n}=\left[\begin{array}{ll}
1 & 1
\end{array}\right]\left[\begin{array}{ll}
\tau_{11} & \tau_{12} \\
\tau_{12} & \tau_{22}
\end{array}\right]\left[\begin{array}{l}
1 \\
1
\end{array}\right]=\left[\begin{array}{ll}
1 & 1
\end{array}\right]\left[\begin{array}{l}
\tau_{11}+\tau_{12} \\
\tau_{12}+\tau_{22}
\end{array}\right]=\tau_{11}+2 \tau_{12}+\tau_{22}
$$

This means we have the off-diagonal terms given by:

$$
\tau_{j k}=\frac{i}{4 \pi} \ln \left[(\underbrace{\lim \frac{1}{T}}_{T \rightarrow \infty} \int_{0}^{T} \theta_{n=j+k}^{ \pm}(t) e^{i\left(\Omega_{j}+\Omega_{k}\right) t} d t)(\underbrace{\lim \frac{1}{T}}_{T \rightarrow \infty} \int_{0}^{T} \theta_{n=-(j+k)}^{ \pm}(t) e^{-i\left(\Omega_{j}+\Omega_{k}\right) t} d t)\right]-\frac{1}{2}\left(\tau_{j j}+\tau_{k k}\right)
$$

Now, we have the Riemann spectrum $\tau, \boldsymbol{\phi}^{ \pm}$in the simplest of terms. This serves as a numerical algorithm for determining the off-diagonal elements of the period matrix. Equation (159) is (154) in different notation.

\subsection{Modeling the Surface Wave Elevation}

Finally, we can construct a wave model to simulate the surface wave elevation $\eta(x, t)$, since we have the Riemann spectrum $\tau, \phi^{ \pm}$. First, we compute the theta functions

$$
\begin{aligned}
\theta\left(x, t \mid \boldsymbol{\tau}, \boldsymbol{\phi}^{ \pm}\right) & =\sum_{n=-\infty}^{\infty} \theta_{n}^{ \pm}(t) e^{i k_{n} x} \\
\theta_{n}^{ \pm}(t) & =\sum_{\mathbf{n} \in \mathbb{Z}} \theta_{\mathbf{n}}^{ \pm} e^{-i \mathbf{n} \cdot \Omega t}, \quad \theta_{\mathbf{n}}^{ \pm}=\delta_{n, I(\mathbf{n} \cdot \mathbf{k})} e^{-i \pi \mathbf{n} \cdot \boldsymbol{\tau} \mathbf{n}+i \mathbf{n} \cdot \boldsymbol{\phi}^{ \pm}}
\end{aligned}
$$


Then, the surface wave elevation is given by:

$$
\Xi(x, t)=a_{o} \frac{\theta\left(x, t \mid \boldsymbol{\tau}, \boldsymbol{\phi}^{-}\right)}{\theta\left(x, t \mid \boldsymbol{\tau}, \boldsymbol{\phi}^{+}\right)} e^{i k_{0} x-i\left(\omega_{0}+\Omega^{\prime}\right) t}
$$

which has the quasiperiodic Fourier series (see Osborne [3]):

$$
\Xi(x, t)=\sum_{\mathbf{n} \in \mathbb{Z}^{N}} \Xi_{\mathbf{n}} e^{i \mathbf{n} \cdot \mathbf{k} x-i \mathbf{n} \cdot \boldsymbol{\omega} t+i \mathbf{n} \cdot \boldsymbol{\Phi}}
$$

where

$$
\begin{aligned}
& \tau_{j j}=\frac{i}{2 \pi} \ln \left[(\underbrace{\lim }_{T \rightarrow \infty} \frac{1}{T} \int_{0}^{T} \theta_{n=j}^{ \pm}(t) e^{i \Omega_{j} t} d t)(\underbrace{\lim }_{T \rightarrow \infty} \frac{1}{T} \int_{0}^{T} \theta_{n=-j}^{ \pm}(t) e^{-i \Omega_{j} t} d t]\right. \\
& \tau_{j k}=\frac{i}{4 \pi} \ln \left[(\underbrace{\lim }_{T \rightarrow \infty} \frac{1}{T} \int_{0}^{T} \theta_{n=j+k}^{ \pm}(t) e^{i\left(\Omega_{j}+\Omega_{k}\right) t} d t)(\underbrace{\lim }_{T \rightarrow \infty} \frac{1}{T} \int_{0}^{T} \theta_{n=-(j+k)}^{ \pm}(t) e^{-i\left(\Omega_{j}+\Omega_{k}\right) t} d t)\right]-\frac{1}{2}\left(\tau_{j j}+\tau_{k k}\right) \\
& \phi_{j}^{ \pm}=-i \ln \left[\frac{(\underbrace{\lim }_{T \rightarrow \infty} \frac{1}{T} \int_{0}^{T} \theta_{n=j}^{ \pm}(t) e^{i \Omega_{j} t} d t)}{\left.(\underbrace{\lim \frac{1}{T} \int_{0}^{T} \theta_{n=-j}^{ \pm}}_{T \rightarrow \infty}(t) e^{-i \Omega_{j} t} d t)\right]}\right.
\end{aligned}
$$

The last five Equations (161)-(164) are our model for nonlinear one-dimensional wave motion. In Figure 15, we give a flow chart that explains all of the numerical steps that one uses to obtain the Riemann spectrum from data. Figure 16 gives a wave model for the subsequent space/time evolution of a nonlinear wave motion. Figure 17 gives a reminder of the structure of the Riemann matrix as used in Figures 15 and 16. One further step needs to be taken: The complex frequencies $\Omega_{j}$ must be computed in the appropriate way as shown in the next subsection. 
Riemann Spectrum for 1+1 NLS from Surface Wave elevation
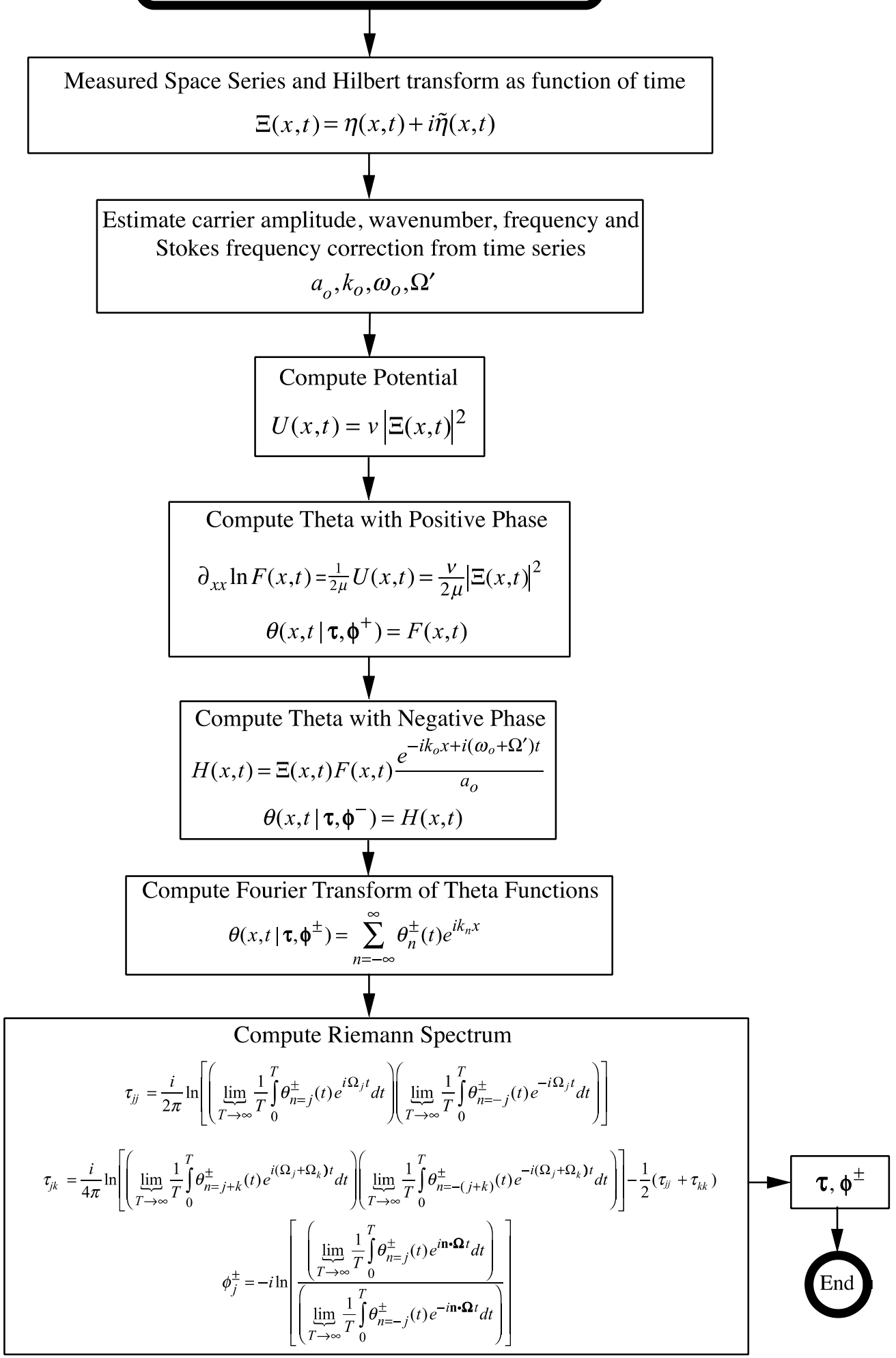

Figure 15. Determine Riemann spectrum $\boldsymbol{\tau}, \boldsymbol{\phi}^{ \pm}$from a wave field $\eta(x, t)$. Notice that in the box labeled "Compute Riemann Spectrum," the frequencies are the usual ones determined with the modulational dispersion relation $\Omega_{j}=\Omega_{j}\left(K_{j}\right)$, which are incommensurable, and therefore require the usual limits over time. 


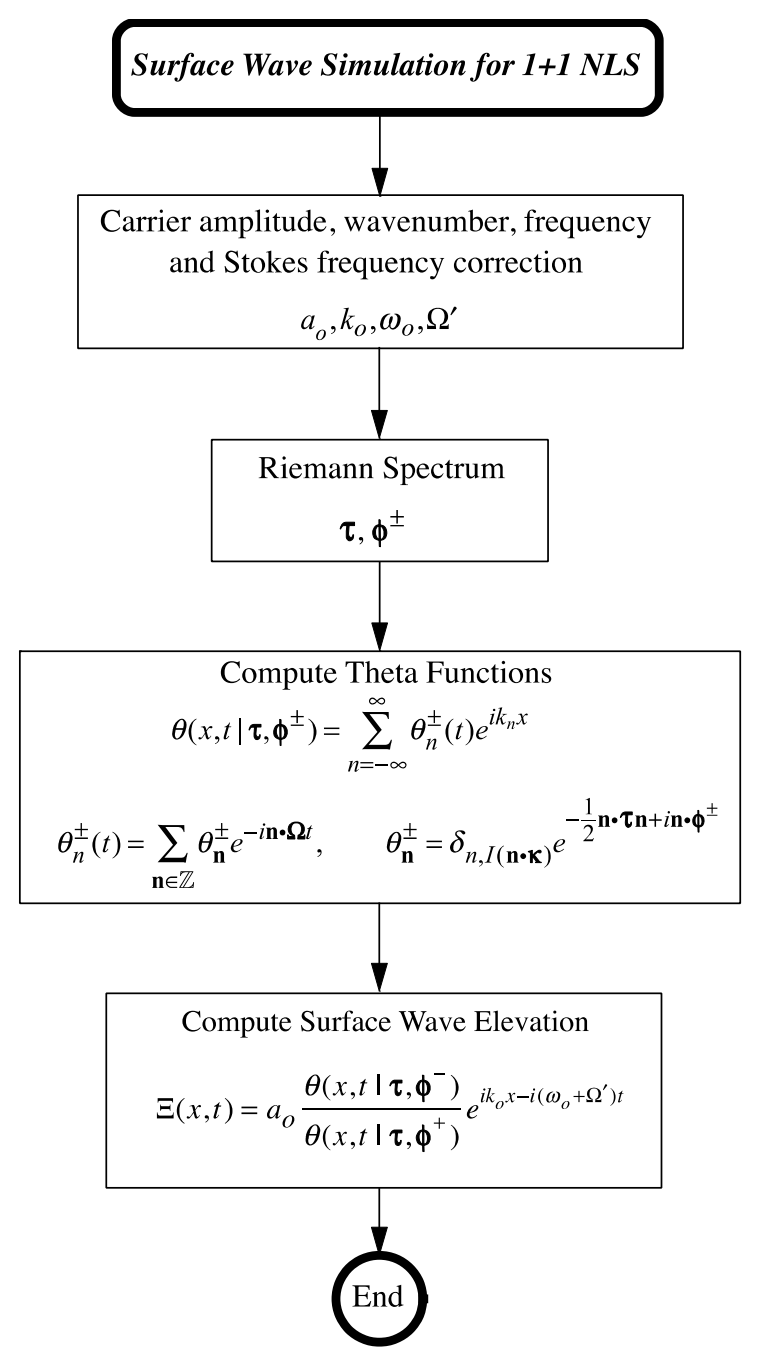

Figure 16. Two-dimensional wave model based on the Riemann spectrum.

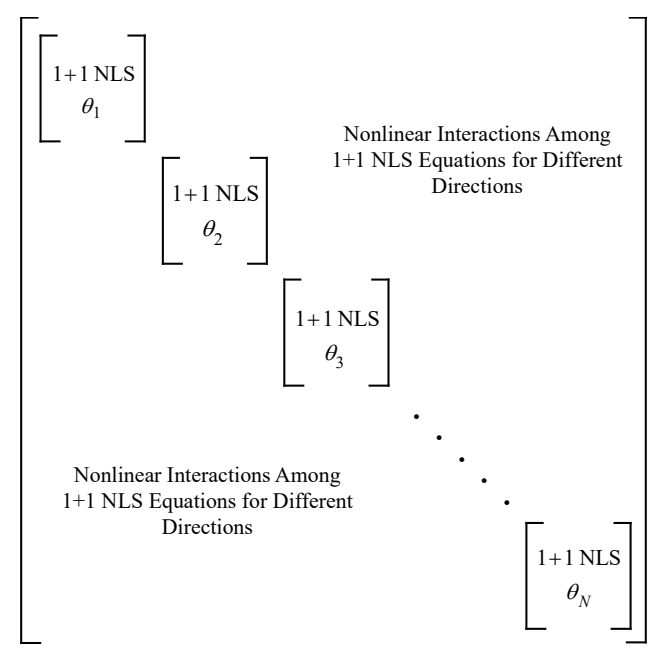

Figure 17. The period matrix for asymptotically solving the $2+1$ NLS equation corresponds to diagonal block matrices for each rotated 1+1 NLS equations for each radial direction in the NLFA spectrum. The off-diagonal elements outside these matrices describe the interactions among the directional $1+1$ NLS equations on the block diagonal and are assumed to be given by rescaled and rotated forms of the first equation in Equation (82) and the second equation in Equation (83). 


\subsection{Computing the Frequencies $\Omega_{j}=\mathbf{n}^{j} \cdot \mathbf{\Omega}$ for Time Series Analysis and Modeling}

The actual quasiperiodic Fourier series frequencies $\Omega_{j}=\mathbf{n}^{j} \cdot \mathbf{\Omega}$ are not the usual real frequencies that occur for linear surface waves and which arise from the well-known dispersion relation: $\omega^{2}=$ $g k \tanh (k h)$. Indeed, under the right circumstances (when (1) the parametric relation $k h>1.363$ holds, essentially for deep enough water, and (2) when the BF parameter $I_{B F}>1$ ) the BF instability comes into play and the frequencies can be complex. This leads, in the quasiperiodic Fourier series themselves, to the formation of breather states and rogue waves. This surprising circumstance needs to be studied in detail as seen in the rest of this subsection, where we focus on results important for time series analysis and for modeling.

\subsubsection{The Linear Model}

For organizational purposes, we begin with the linear model, in order to present an overview of the problem at hand. We now write the 2D Fourier transform in terms of wavenumbers $k_{m}, l_{n}$ so that we have separate indices $m, n$ for the $x$ and $y$ wavenumbers:

$$
\eta(x, y, t)=\sum_{m=-\infty}^{\infty} \sum_{n=-\infty}^{\infty} \eta_{m n} e^{i k_{m} x+i l_{n} y-i \omega_{m n} t+i \phi_{m n}}
$$

where the frequencies, to leading order, are given by the linear dispersion relation:

$$
\omega_{m n}^{2}=\omega_{m n}^{2}\left(k_{m}, l_{n}\right)=g k_{m n} \tanh k_{m n} h, \quad k_{m n}=\sqrt{k_{m}^{2}+l_{n}^{2}}
$$

In the linear model, the frequencies are always real. The wave domain is discrete with $x$ and $y$ coordinates $\left(x_{m}, y_{n}\right)$ for $m=1,2 \ldots M, n=1,2 \ldots N$. The space/time surface wave elevation $\eta(x, y, t)$ is taken from Equation (165) and would look something like that in Figure 18 at time $t=0$. The spectral domain is also discrete. A pictogram of the Fourier transform of a wave field is shown in Figure 19. Of course, the linear model is the standard way to treat remotely sensed data.

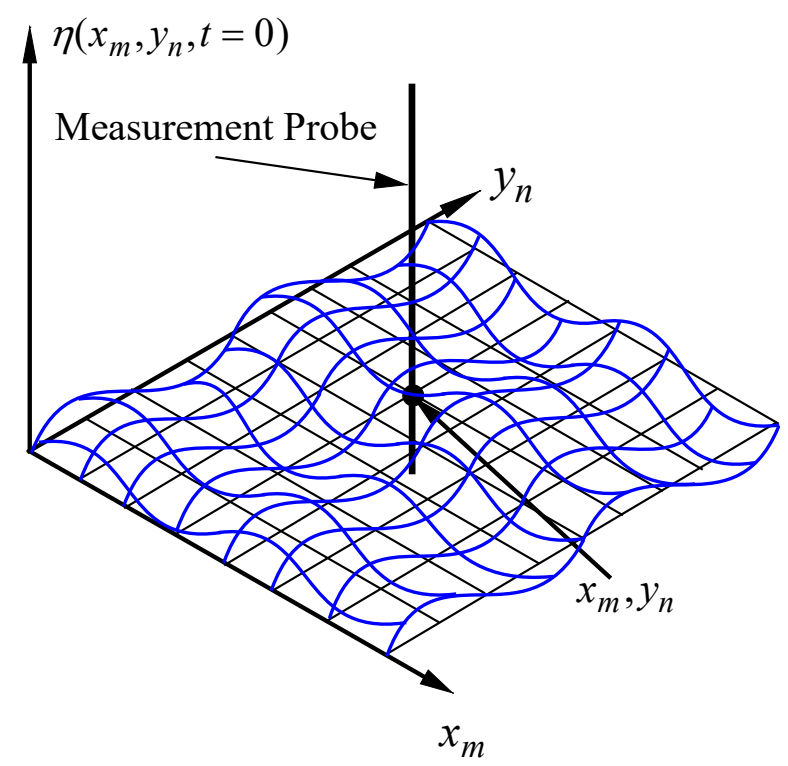

Figure 18. Ocean surface wave elevation in two dimensions at an instant in time, $\eta\left(x_{m}, y_{n}, t=0\right)$, on a grid of coordinate points $\left(x_{m}, y_{n}\right)$, with spatial resolution $(\Delta x, \Delta y)$. 


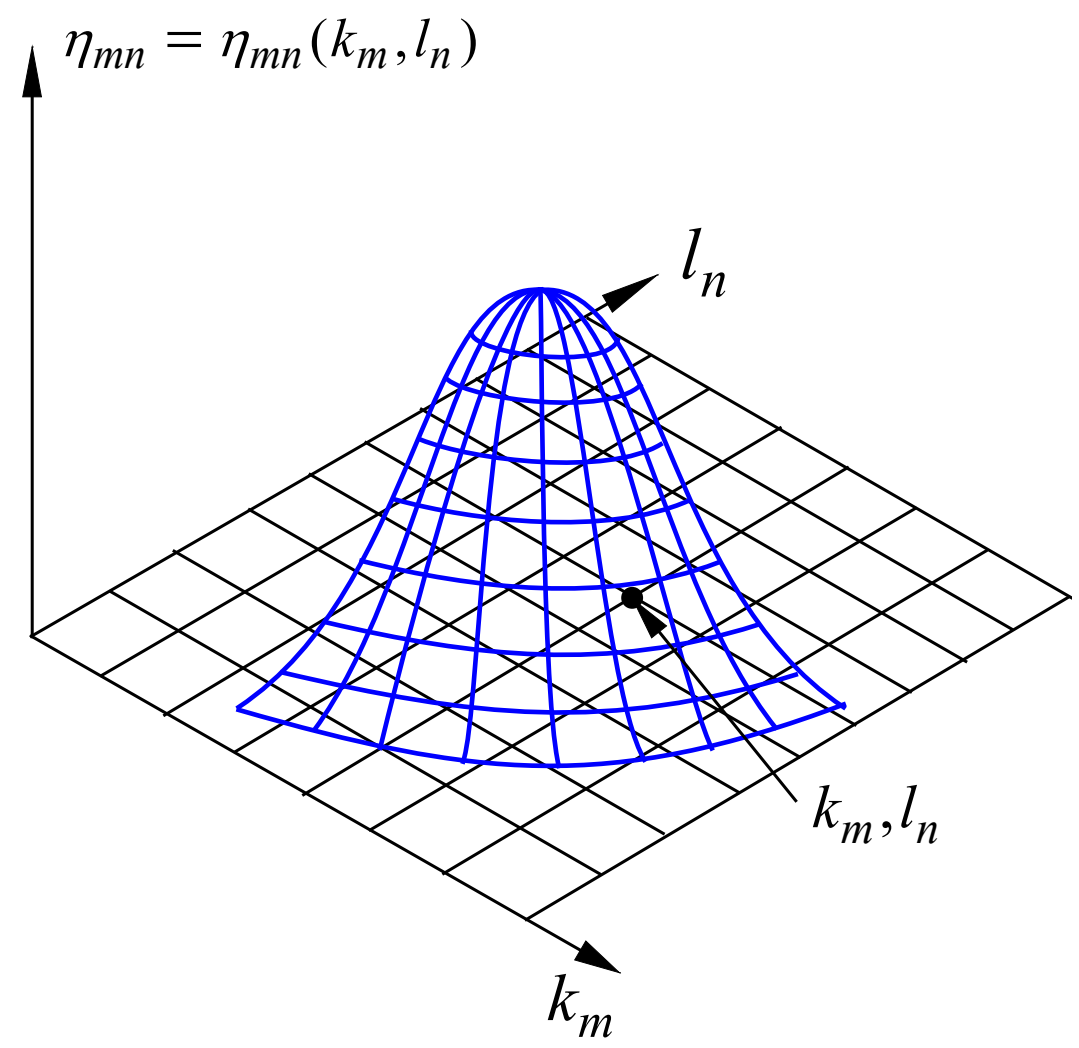

Figure 19. Two-dimensional ocean surface wave spectrum at an instant in time, $\eta_{m n}\left(k_{m}, l_{n}, t=0\right)$, on a commensurable wavenumber grid $\left(k_{m}, l_{n}\right)$ with wavenumber resolution $(\Delta k, \Delta l)$.

We could think of taking a snapshot of the sea surface to obtain an instantaneous discrete wave field, at time $t=0$ say, by any of several methods: radar, lidar, synthetic aperture radar, etc. Then, we can take the 2D FFT of the wave field to obtain the spectrum $\eta_{m n}\left(k_{m}, l_{n}\right)$, and the power spectrum $P\left(k_{m}, l_{n}\right)=\left|\eta_{m n}\left(k_{m}, l_{n}\right)\right|^{2}$. Then, Equation (165) has the form:

$$
\eta(x, y, t=0)=\sum_{m=-\infty}^{\infty} \sum_{n=-\infty}^{\infty} \eta_{m n} e^{i k_{m} x+i l_{n} y+i \phi_{m n}}
$$

If, on the other hand, we measure a time series at a fixed spatial point $x=y=0$, we would write:

$$
\eta(0,0, t)=\sum_{m=-\infty}^{\infty} \sum_{n=-\infty}^{\infty} \eta_{m n} e^{-i \omega_{m n} t+i \phi_{m n}}
$$

In Figure 20, we show the directional spectrum in the upper panel, and, in the lower panel, we show the frequency spectrum, where each frequency $\omega_{m n}$ is given by Equation (166) to leading order. The irregular frequencies that are incommensurable in the bottom of Figure 20 reflect higher order effects discussed in Equation (170) below. Thus, the wavenumbers are here assumed commensurable (proportional to the integers) and the frequencies are not commensurable, i.e., they are not equally spaced. Furthermore, there is a unique mapping between each wavenumber pair $k_{m}, l_{n}$ and the frequency $\omega_{m n}$ given to leading order by (166) and to the next order by Equation (170). Hence, each frequency $\omega_{m n}$ is associated with the wavenumber pair $k_{m}, l_{n}$ and, therefore with a direction in the directional spectrum

$$
\tan \theta_{m n}=\frac{k_{m}}{l_{n}}
$$




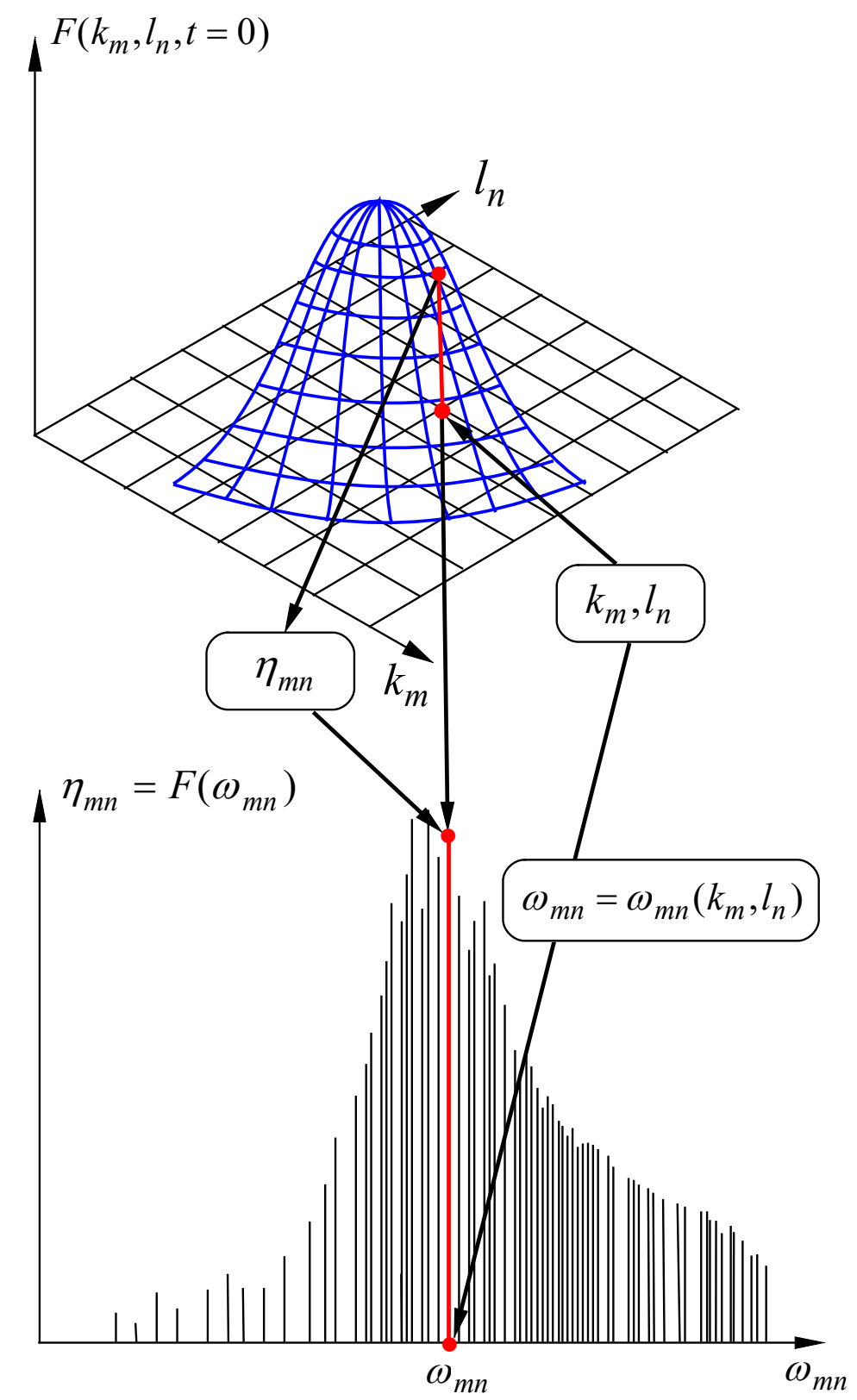

Figure 20. Two-dimensional ocean surface wave spectrum at an instant in time, $F\left(k_{m}, l_{n}, t=0\right)=$ $\eta_{m n}\left(k_{m}, l_{n}\right)$ on a wavenumber grid $\left(k_{m}, l_{n}\right)$, which is commensurable with wavenumber resolution $(\Delta k, \Delta l)$ (upper panel) and the associated frequency spectrum with incommensurable frequencies (lower panel). Every wavenumber pair $\left(k_{m}, l_{n}\right)$ (upper panel) maps to the frequency $\omega_{m n}=\omega_{m n}\left(k_{m}, l_{n}\right)$ (via the dispersion relation, lower panel): Therefore, the frequencies $\omega_{m n}=\omega_{m n}\left(k_{m}, l_{n}\right)$ are incommensurable and each is uniquely associated with the spectral amplitudes $\eta_{m n}$ in Equations (165), (166) and (170).

Thus, (168), together with (166) (or (170)), allows us to determine the directional spectrum, provided we use the incommensurable frequencies $\omega_{m n}$. In the traditional analysis of time series, we use commensurable frequencies $\omega_{i}$, which derives from $1 / T$ where $T$ is the length of a measured time series: We do not here follow this tradition. We instead see that each point in a particular power spectrum $P\left(k_{m}, l_{n}\right) \equiv P_{m n}$ is associated with a particular wavenumber pair $\left(k_{m}, l_{n}\right)$, which through (166) is associated with a particular frequency $\omega_{m n}$ and direction $\theta_{m n}$ :

$$
\omega_{m n} \Rightarrow\left(k_{m}, l_{n}\right) \Rightarrow \theta_{m n}
$$


Therefore, we see that we can get an estimate of the directional spectrum from a time series (from (168), provided of course that (166), the dispersion relation, has sufficient accuracy to give us the actual incommensurable frequencies $\omega_{m n}$ we need: Obtaining the directional spectrum from a time series is the focus of another paper. The focus of this subsection is to provide a basis for constructing the appropriate incommensurable frequencies for space/time series analysis. In particular, we address the influence of the BF instability on the dispersion relation.

It may be necessary, under certain circumstances, to extend the order of the dispersion relation, see Whitham [60], page 474:

$$
\omega^{2}=g k \tanh k h\left[1+\left(\frac{9 \tanh ^{4}(k h)-10 \tanh ^{2}(k h)+9}{8 \tanh ^{4}(k h)}\right) k^{2} a^{2}+\ldots\right]
$$

where

$$
\mu_{2}=\frac{1}{2} k \operatorname{coth} k h\left(1+\frac{3}{2 \sinh ^{2}(k h)}\right)
$$

and the Stokes wave is given by

$$
\eta=a \cos (k x-\omega t)+\mu_{2} a^{2} \cos 2(k x-\omega t)+\ldots
$$

Note that the dispersion relation above (170) reduces in shallow water to

$$
\frac{\omega}{c_{0} k}=1-\frac{h^{2} k^{2}}{6}+\frac{9 a^{2}}{16 h^{4} k^{2}}+\ldots
$$

a result already known for the KdV equation (Whitham [60] Equation (13.119), page 473). In deep water, we obtain for (170)

$$
\omega^{2}=g k\left(1+k^{2} a^{2}+\ldots\right)
$$

which is the original Stokes result (Whitham [60] Equation (13.124), page 475).

Thus, an alternative is to use the second order Stokes dispersion relation (170) (for arbitrary water depth $h$ ) in our analysis instead of (166). Of course, we could extend this to arbitrary order and include interactions as well. All of these arguments, for arbitrary depth, are handled very elegantly in the paper by Hasimoto and Ono [48] for the one-dimensional case of the nonlinear Schrödinger equation. The irregular nature of the frequencies in Figure 20 reflects the incommensurability in water wave dispersion.

\subsubsection{Linear Schrödinger Equation in 1+1 Dimensions}

We now need to first look at the solution to the linear Schrödinger equation (LSE):

$$
i\left(\psi_{t}+\omega^{\prime} \psi_{x}\right)+\frac{1}{2} \omega^{\prime \prime} \psi_{x x}=i\left(\psi_{t}+\frac{\omega_{o}}{2 k_{o}} \psi_{x}\right)-\frac{\omega_{o}}{8 k_{o}^{2}} \psi_{x x}=0
$$

where from $\omega^{2}=g k$ we have:

$$
C_{g}=\omega^{\prime}=\frac{\omega_{o}}{2 k_{o}} \quad \mu=\frac{1}{2} \omega^{\prime \prime}=-\frac{\omega_{o}}{4 k_{o}^{2}}
$$

The general coefficients for arbitrary depth occur for $\omega^{2}=g k \tanh (k h)$ and are given by Hasimoto and Ono [48]. We know that the dispersion relation for the carrier wave described by the linear Schrödinger equation is given from a plane wave solution

$$
\psi(x, t)=\psi_{0} e^{i K x-\Omega t}
$$


for which we obtain

$$
\Omega(K)=\omega^{\prime} K+\frac{1}{2} \omega^{\prime \prime} K^{2}=C_{g} K+\frac{1}{2} \omega^{\prime \prime} K^{2}
$$

We should remember that this is an expansion of the deep-water dispersion relation $\omega^{2}=g k$ provided we add back in the carrier to get the surface elevation

$$
\Xi(x, t)=\psi(x, t) e^{i k_{0} x-i \omega_{0} t}=\psi_{0} e^{i K x-\Omega t} e^{i k_{0} x-i \omega_{0} t}=\psi_{0} e^{i\left(k_{0}+K\right) x-\left(\omega_{0}+\Omega\right) t}
$$

Put in the dispersion relation (177) to give

$$
\begin{aligned}
\Xi(x, t) & =\psi_{0} e^{i\left(k_{0}+K\right) x-\left(\omega_{0}+\Omega\right) t}=\psi_{0} e^{i k x-\omega t} \\
k & =k_{0}+K \\
\omega & =\omega_{0}+\Omega
\end{aligned}
$$

Thus, we have finally the dispersion relation for the surface elevation:

$$
\omega=\omega_{0}+\Omega(K)=\omega_{0}+\omega^{\prime} K+\frac{1}{2} \omega^{\prime \prime} K^{2}=\omega_{o}+C_{g} K+\mu K^{2}
$$

By writing this expression about the carrier wavenumber and frequency $k_{0}, \omega_{0},(180)$ can be written in terms of the physically useful frequency-wavenumber pair $\omega, k$ :

$$
\omega=\omega(k)=\omega_{o}+\Omega(K)=\omega_{o}+\omega^{\prime}\left(k-k_{o}\right)+\frac{1}{2} \omega^{\prime \prime}\left(k-k_{0}\right)^{2}=\omega_{o}+C_{g}\left(k-k_{0}\right)+\mu\left(k-k_{o}\right)^{2}
$$

This expression is then the dispersion relation $\omega=\sqrt{g k}$ in disguise, i.e., it has been written as a Taylor series expansion (180)' about $k=k_{0}$. A graph comparing $\omega=\sqrt{g k}$ with the Taylor series expansion (180)' is given in Figure 21 below. We see that the two agree with each other rather well around the peak of the spectrum. (To improve agreement, we would need to extend the order of the LSE Equation (175), something we do in a later paper, where we use full linear dispersion.) We see that a narrow-banded spectrum $F\left(k-k_{0}\right)$ centered about $k_{0}$ has a dispersion relation expanded about zero that is the brown curve, $\omega=\omega\left(k-k_{o}\right)$. The actual dispersion relation $\omega=\sqrt{g k}$ is shown as a blue curve. Why go through all of this approximation for the dispersion relation? Because at linear order we have the LSE which has a Fourier series solution, which is what we seek. Thus, a Taylor series expansion for the dispersion relation gives us the LSE (175). Furthermore, by adding the appropriate nonlinear term, the LSE becomes the nonlinear Schrödinger equation (NLS), which describes the BF instability and the imaginary correction to the dispersion relation (180). With this BF correction, we will be able to analyze time series for breathers and rogue waves.

Another graph of the polynomial dispersion relation (180) is shown in Figure 22. We write this in the following way for clarity:

$$
\omega(k)=\omega_{o}+C_{g}\left(k-k_{o}\right)+\mu\left(k-k_{o}\right)^{2}
$$

In Figure 22, we have evenly spaced wavenumbers (horizontally) and unequally spaced (incommensurable) frequencies corresponding to the horizontal lines. The frequencies are more closely spaced above, with larger spacings below. A more conventional view is given in Figure 23, where we see that for LSE (175) motion the frequencies are denser to the right and less dense to the left of the spectrum. 


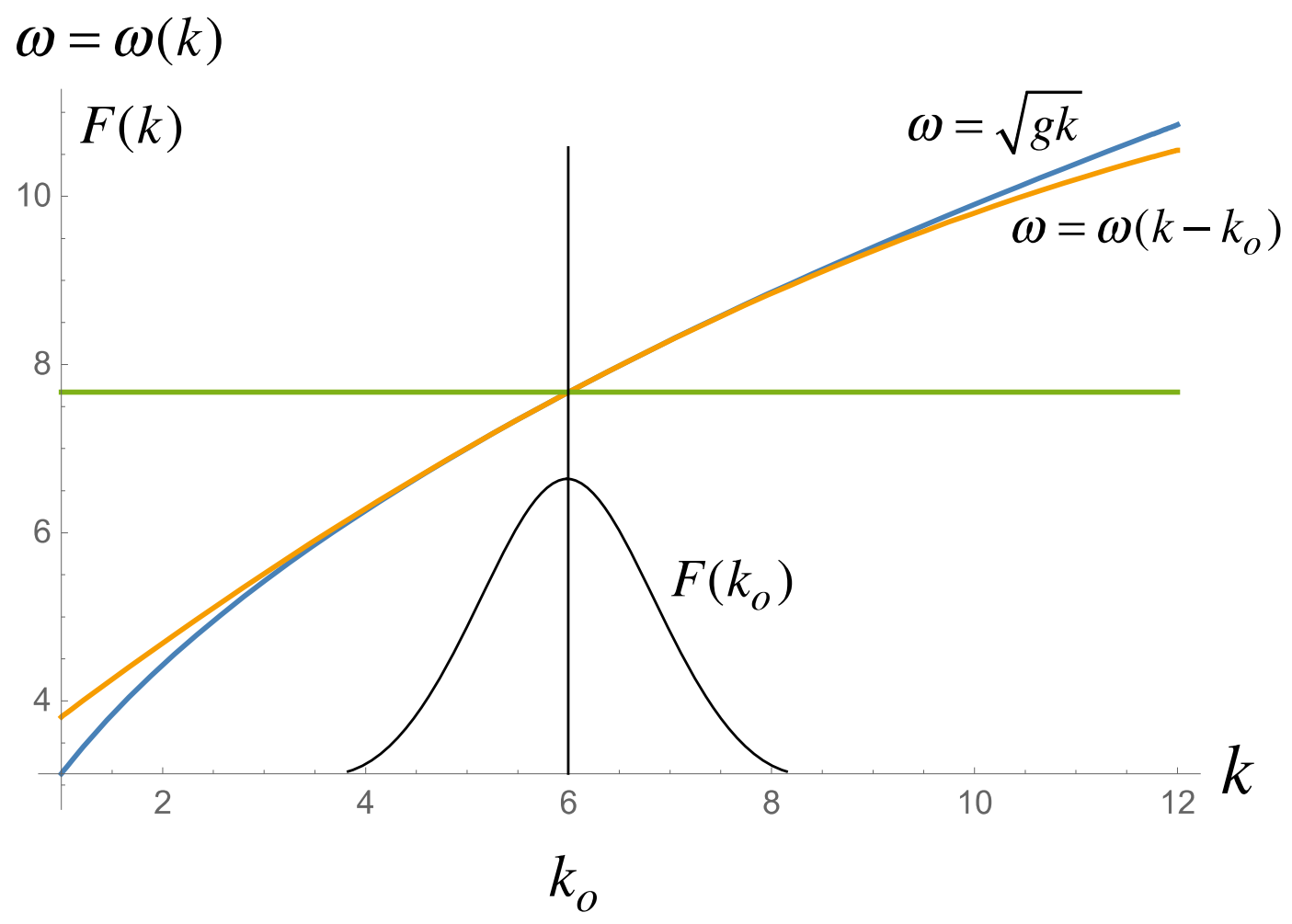

Figure 21. Comparison of linear, deep-water dispersion $\omega=\sqrt{g k}$, (blue) with the dispersion relation of the 1+1 NLS Equation (181) (brown). The two agree rather well with each other near the peak of the spectrum.

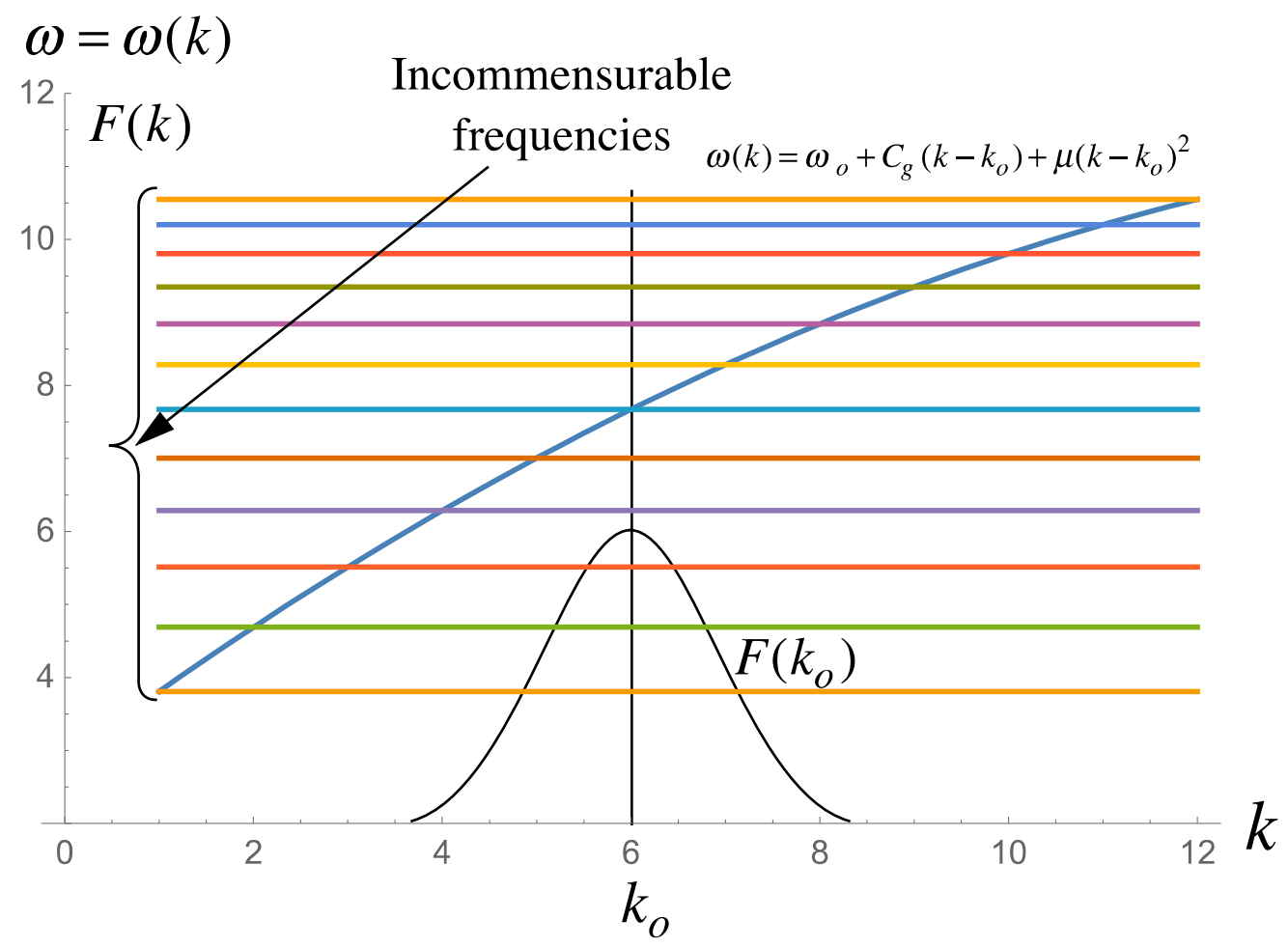

Figure 22. Incommensurable frequencies as a function of wavenumber for the 1+1 NLS Euqation (175). 


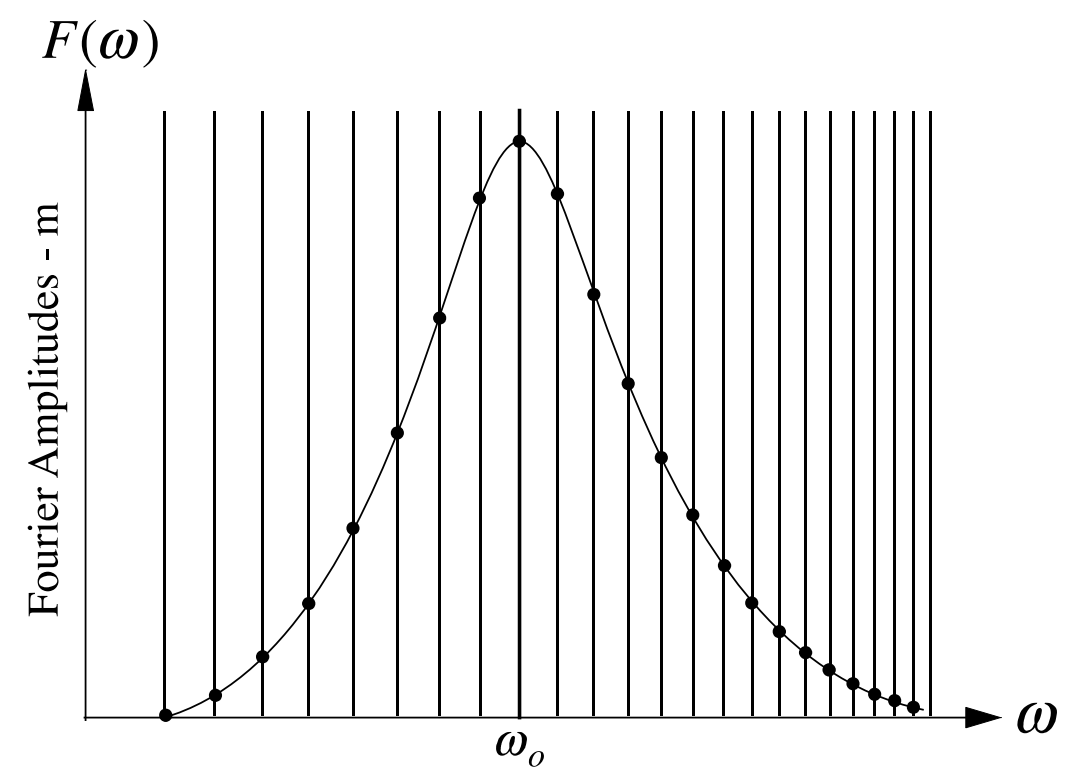

Incommensurable Frequencies - $\mathrm{Hz}$

Figure 23. A Fourier spectrum of the 1+1 LSE equation with incommensurable frequencies given by the dispersion relation (181).

\subsubsection{The Modulational Instability for $1+1$ NLS}

We now seek to find the correction to the dispersion relation (180)' for the nonlinear Schrödinger equation in $1+1$ dimensions ( $1+1$ NLS) (15). The modulational dispersion relation for $1+1$ NLS is given by (Osborne [3]):

$$
\Omega= \pm \mu K \sqrt{K^{2}-\frac{2 v a_{o}^{2}}{\mu}}
$$

Thus, a small modulation of a Stokes wave is unstable if $K^{2}<2 a_{0}^{2} v / \mu$ because the frequency $\Omega$ will then be imaginary. If we turn off the parameter $v\left(\operatorname{set} v=0\right.$ ), we have $\Omega=\mu K^{2}$, a stable condition, already discussed for the linear problem in (180)' above.

Now if there is no nonlinearity, and therefore no BF instability, the nonlinear term (proportional to $v$, see also (15)) in (182) can be left out to obtain:

$$
\Omega \simeq \pm \mu K^{2}
$$

This leads to the expression for the surface elevation

$$
\omega=\omega_{o}+\Omega \simeq \omega_{o} \pm \mu\left(k-k_{o}\right)^{2}
$$

where in deep water

$$
\mu=\frac{1}{2} \omega^{\prime \prime}=-\frac{\omega_{o}}{4 k_{o}^{2}}
$$

In addition, notice that we can neglect the minus sign, in order to get:

$$
\omega=\omega_{o}+\Omega \simeq \omega_{o}+\mu\left(k-k_{o}\right)^{2}
$$


This is the linearized dispersion relation as derived from the modulational instability in the linear limit. With nonlinearity, we have

$$
\omega=\omega_{o}+\Omega=\omega_{0}+\mu K \sqrt{K^{2}-\frac{2 a_{o}^{2} v}{\mu}}
$$

What has been left out? The group speed, for indeed this is the result when we remove the group speed term from NLS by a Galilean transformation, and so we have: Adding back in the group speed leads to the expression:

$$
\omega=\omega_{o}+C_{g} K+\mu K \sqrt{K^{2}-\frac{2 a_{o}^{2} v}{\mu}}
$$

Then, use $K=k-k_{o}$ to get:

$$
\omega=\omega_{o}+\Omega=\omega_{o}+C_{g}\left(k-k_{o}\right)+\mu\left(k-k_{o}\right) \sqrt{\left(k-k_{o}\right)^{2}-\frac{2 a_{o}^{2} v}{\mu}}
$$

Thus, finally, if we again leave out the nonlinear piece with the nonlinear coefficient $v$, we get the linear result

$$
\omega=\omega_{0}+\Omega=\omega_{0}+C_{g}\left(k-k_{o}\right)+\mu\left(k-k_{o}\right)^{2}
$$

Equation (189), finally, is the actual dispersion relation we seek. This gives us the full dispersion for the 1+1 NLS equation, with the modulational instability, in a linear quasiperiodic Fourier series (161)'. Equation (189) is the fundamental frequency that appears in (161)-(164), our equations for water wave modeling.

What Equation (189) says is that, as long as the $v$ term (second term under the square root) is small enough, the frequency is real. However, if the BF parameter is large enough, the square root goes negative (because the $v$ term exceeds the squared wavenumber term in the square root), and we get an imaginary contribution to the dispersion relation. Thus, most generally, we have a complex dispersion relation (with real and imaginary parts). This will happen when we are near the peak of the spectrum, inside the band of instability, where the breathers live (see Osborne et al. [5] for details in the analysis of data).

Of course, we can rewrite (189) in a slightly different form by noting that (182) can be written

$$
\Omega= \pm \mu K^{2} \sqrt{1-\frac{2 v a_{o}^{2}}{\mu K^{2}}}= \pm \mu K^{2} \sqrt{1-I_{B F^{\prime}}^{2}} \quad I_{B F}=\sqrt{\frac{2 v}{\mu}} \frac{a_{o}}{K} \simeq \frac{2 \sqrt{2} k_{o}^{2} a_{o}}{K}
$$

where the second term for the BF parameter $I_{B F}$ is for deep water; the first term is for arbitrary depth. This gives for (189):

$$
\omega=\omega_{o}+\Omega=\omega_{0}+C_{g}\left(k-k_{o}\right)+\mu\left(k-k_{o}\right)^{2} \sqrt{1-\frac{2 v a_{o}^{2}}{\mu\left(k-k_{o}\right)^{2}}}
$$

or

$$
\omega=\omega_{0}+\Omega=\omega_{o}+C_{g}\left(k-k_{o}\right)+\mu\left(k-k_{o}\right)^{2} \sqrt{1-I_{B F}^{2}}
$$

where the spectral BF parameter is

$$
I_{B F}=\sqrt{\frac{2 v}{\mu}} \frac{a_{o}}{\left(k-k_{o}\right)} \simeq \frac{2 \sqrt{2} k_{o}^{2} a_{o}}{\left(k-k_{o}\right)}
$$


This latter expression for $I_{B F}$ means that, as we move away from the peak of the spectrum, we will see the BF parameter decrease (as $k$ increases). $I_{B F}$ formally goes to infinity at the peak of the spectrum. As we move away from the peak of the spectrum $I_{B F}$ decreases and, eventually, falls below 1 , and we will no longer have an unstable condition. The condition $I_{B F}<1$ means that we will have a band of spectrum $\left(f_{o} \pm I_{B F} \Delta f\right.$, where $f_{o}=\omega_{o} / 2 \pi$ and $\Delta f$ is the frequency resolution, see Osborne [1]) around the peak, inside of which we have unstable "breather-type" Fourier modes, and outside of which the waves are stable Stokes waves. This just demonstrates that breathers live near the peak of the spectrum (see Figure 1 and further discussion in Osborne [1], Chapter 29). Recall that we can use the depth dependent coefficients for $C_{g}, \mu, \quad v$ in the above formulas to be consistent with the depth dependent dispersion relation $\omega=g k \tanh k h$ or its nonlinear extension (170). In this way, we can compute (193) and (194) for arbitrary depth.

Finally, we can add the nonlinear Stokes wave correction, to obtain, where we use $\omega_{o}=\omega_{p}$ and $k_{o}=k_{p}$

$$
\omega_{n}=\omega_{p}+\Omega=\omega_{p}+C_{g}\left(k_{n}-k_{p}\right)+\mu\left(k_{n}-k_{p}\right)^{2} \sqrt{1-I_{B F}^{2}}+v a_{o}^{2}
$$

where $n=1,2 \ldots N$ and $N$ is the number of Fourier modes. Note that (195) is equivalent to (193) except for the nonlinear Stokes correction. Equation (195) is to be used in (161)' for time series analysis and numerical modeling. Likewise, the frequencies $\Omega_{j}$ used in Figure 16 are to be computed by the results in this section.

\subsubsection{LSE in $2+1$ Dimensions}

We now need to look at the solution to the $2+1$ linear Schrödinger equation (2+1 LSE).

$$
i\left(\psi_{t}+\omega^{\prime} \psi_{x}\right)+\frac{1}{2} \omega^{\prime \prime} \psi_{x x}-\omega^{\prime \prime} \psi_{y y}=i\left(\psi_{t}+\frac{\omega_{o}}{2 k_{o}} \psi_{x}\right)-\frac{\omega_{o}}{8 k_{o}^{2}} \psi_{x x}+\frac{\omega_{o}}{4 k_{o}^{2}} \psi_{y y}=0
$$

where the prime (') represents the derivative with respect to wavenumber and the linear, deep water dispersion relation is $\omega^{2}=g k$. We then have for deep water:

$$
C_{g}=\omega^{\prime}=\frac{\omega_{0}}{2 k_{o}} \mu=\frac{1}{2} \omega^{\prime \prime}=-\frac{\omega_{o}}{8 k_{o}^{2}} \rho=\omega^{\prime \prime}=-\frac{\omega_{o}}{4 k_{o}^{2}}
$$

Then, the 2+1 LSE has the form:

$$
i\left(\psi_{t}+\omega^{\prime} \psi_{x}\right)+\mu \psi_{x x}-\rho \psi_{y y}=0
$$

Thus, we see that the dispersion relation for the carrier wave described by the linear Schrödinger equation is given from a plane wave solution

$$
\psi(x, y, t)=\psi_{0} e^{i K_{x} x+i K_{y} y-\Omega t}
$$

for which we obtain the modulational frequency

$$
\Omega\left(K_{x}, K_{y}\right)=\omega^{\prime} K_{x}+\frac{1}{2} \omega^{\prime \prime} K_{x}^{2}-\omega^{\prime \prime} K_{y}^{2}=C_{g} K+\frac{1}{2} \omega^{\prime \prime} K_{x}^{2}-\omega^{\prime \prime} K_{y}^{2}
$$

We should remember that this is an expansion in 2D of the deep-water dispersion relation $\omega^{2}=g k$ provided we add back in the carrier $\omega_{0}$. To get the surface elevation,

$$
\Xi(x, y, t)=\psi(x, y, t) e^{i k_{0} x-i \omega_{0} t}=\psi_{0} e^{i K_{x} x+i K_{y} y-\Omega t} e^{i k_{0} x-i \omega_{0} t}=\psi_{0} e^{i\left(k_{0}+K_{x}\right) x+i K_{y} y-\left(\omega_{0}+\Omega^{\prime}\right) t}
$$


Put in the dispersion relation to get the surface elevation:

$$
\begin{aligned}
\Xi(x, y, t) & =\psi_{0} e^{i\left(k_{0}+K_{x}\right) x+i K_{y} y-\left(\omega_{0}+\Omega\right) t}=\psi_{0} e^{i k x+i l y-\omega t} \\
k & =k_{o}+K_{x} \\
l & =K_{y} \\
\omega & =\omega_{o}+\Omega
\end{aligned}
$$

Thus, we have finally the dispersion relation, truncated at the order of the $2+1$ LSE, which generally is a Taylor series expansion of $\omega^{2}=g k \tanh k h$ :

$$
\omega=\omega_{o}+\Omega\left(K_{x}, K_{y}\right)=\omega_{0}+\omega^{\prime} K_{x}+\frac{1}{2} \omega^{\prime \prime} K_{x}^{2}-\omega^{\prime \prime} K_{y}^{2}=\omega_{o}+C_{g} K+\frac{1}{2} \omega^{\prime \prime} K_{x}^{2}-\omega^{\prime \prime} K_{y}^{2}
$$

By making an expansion (about the carrier $k_{0}, \omega_{0}$ ), we have the dispersion relation for the 2+1 LSE (198):

$$
\begin{aligned}
\omega & =\omega_{o}+\Omega(k, l)=\omega_{0}+\omega^{\prime}\left(k-k_{o}\right)+\frac{1}{2} \omega^{\prime \prime}\left(k-k_{o}\right)^{2}-\omega^{\prime \prime} l^{2}= \\
& =\omega_{o}+C_{g}\left(k-k_{0}\right)+\frac{1}{2} \omega^{\prime \prime}\left(k-k_{o}\right)^{2}-\omega^{\prime \prime} l^{2}
\end{aligned}
$$

This latter expression (203) is then the dispersion relation

$$
\omega^{2}=g \kappa=g \sqrt{k^{2}+l^{2}}
$$

in disguise, i.e., it is a Taylor series expansion (203) of the dispersion relation (204), consistent with the 2+1 LSE (198).

7.11.5. The Modulational Instability in 2D

This is the modulational dispersion relation for the $2+1$ NLS equation:

$$
\Omega= \pm \mu \kappa \sqrt{\kappa^{2}-\frac{2 a_{o}^{2} v}{\mu}}, \quad \kappa=\sqrt{K_{x}^{2}-2 K_{y}^{2}}
$$

Thus, a small modulation of a Stokes wave is unstable if $\kappa^{2}<2 a_{0}^{2} v / \mu$ because the frequency $\Omega$ will be imaginary. If we turn off the nonlinearity $v\left(\operatorname{set} v=0\right.$ ), we have $\Omega=\mu \kappa^{2}$, a stable condition. If there is no nonlinearity, and therefore no BF instability, the nonlinear term $v$ must be left out:

$$
\Omega \simeq \pm \mu \kappa^{2}= \pm \mu\left(K_{x}^{2}-2 K_{y}^{2}\right)
$$

This leads to the linear limit for the dispersion relation for the surface elevation

$$
\omega=\omega_{0}+C_{g}\left(k-k_{o}\right)+\frac{1}{2} \omega^{\prime \prime}\left(k-k_{o}\right)^{2}-\omega^{\prime \prime} l^{2}
$$

where

$$
\mu=\frac{1}{2} \omega^{\prime \prime}=-\frac{\omega_{o}}{4 k_{o}^{2}}
$$

Now, add back in the group speed term for $2+1$ NLS and get the full expression

$$
\omega=\omega_{o}+\Omega=\omega_{o}+C_{g} K_{x}+\mu \kappa \sqrt{\kappa^{2}-\frac{2 a_{o}^{2} v}{\mu}}
$$


for

$$
\kappa=\sqrt{K_{x}^{2}-2 K_{y}^{2}}=\sqrt{\left(k-k_{o}\right)^{2}-2 l^{2}}
$$

Then, use $K_{x}=k-k_{0}, K_{y}=l$ to get:

$$
\omega=\omega_{0}+\Omega=\omega_{0}+C_{g} K_{x}+\mu \kappa \sqrt{\kappa^{2}-\frac{2 a_{o}^{2} v}{\mu}}, \quad \kappa=\sqrt{\left(k-k_{o}\right)^{2}-2 l^{2}}
$$

Thus, finally, if we toss the nonlinear piece, $v$, we again get (207):

$$
\omega=\omega_{0}+\Omega=\omega_{0}+C_{g}\left(k-k_{0}\right)+\mu\left[\left(k-k_{0}\right)^{2}-2 l^{2}\right]
$$

Equation (211), finally, is the actual $2+1$ dispersion relation we want for two-dimensional wave motion. This gives us the full dispersion, with the modulational instability, in a linear quasiperiodic Fourier series. This will happen when we are near the peak of the spectrum, inside the band of instability (the cyan region of Figure 4), where the breathers live.

It is important to notice that $\kappa=\left[\left(k-k_{0}\right)^{2}-2 l^{2}\right]^{1 / 2}$, but one might physically expect that we should have a plus sign instead of a minus sign under the square root. The minus sign occurs because there is a minus sign in $2+1$ NLS, i.e., there is a minus sign in front of the second derivative with respect to $y$. Thus, $2+1$ NLS is hyperbolic for water waves, not elliptic as in quantum mechanics.

We can write $2 \mathrm{D}$ dispersion in terms of the BF instability parameter by writing:

$$
\omega=\omega_{o}+\Omega=\omega_{o}+C_{g} K_{x}+\mu \kappa^{2} \sqrt{1-\frac{2 a_{o}^{2} v}{\mu \kappa^{2}}}, \quad \kappa=\sqrt{\left(k-k_{o}\right)^{2}-2 l^{2}}
$$

We then obtain the final result:

$$
\omega=\omega_{0}+\Omega=\omega_{0}+C_{g}\left(k-k_{0}\right)+\mu \kappa^{2} \sqrt{1-I_{B F 2^{\prime}}^{2}} \quad \kappa=\sqrt{\left(k-k_{o}\right)^{2}-2 l^{2}}
$$

where the $2 \mathrm{D}$ spectral $\mathrm{BF}$ parameter is

$$
I_{B F 2}=\sqrt{\frac{2 v}{\mu}} \frac{a_{o}}{\kappa} \simeq \frac{2 \sqrt{2} k_{o}^{2} a_{o}}{\kappa}
$$

This latter expression means that, as we move away from the peak of the spectrum, we will see the BF parameter decrease as $1 / \kappa$. One should be aware, however, that the instability about the peak of the spectrum lies in the cyan colored zone of Figure 4 . At some point, $I_{B F 2}$ will fall below 1 , and we will no longer be unstable. The condition $I_{B F 2}>1$ means that we will have a band of spectrum around the peak, defined by the cyan zone of Figure 4, inside of which we have unstable "breather-type" conditions, and outside of which the waves are stable Stokes waves. Recall that we can use the depth dependent coefficients for $C_{g}, \mu, v$ in the above formulas to be consistent with the depth dependent dispersion relation $\omega=g k \tanh k h$.

Finally, we can add the Stokes wave correction:

$$
\omega=\omega_{o}+\Omega=\omega_{o}+C_{g}\left(k-k_{o}\right)+\mu \kappa^{2} \sqrt{1-I_{B F 2}^{2}}+v a_{o}^{2}, \quad \kappa=\sqrt{\left(k-k_{o}\right)^{2}-2 l^{2}}
$$

where

$$
\Omega=C_{g}\left(k-k_{o}\right)+\mu \kappa^{2} \sqrt{1-I_{B F 2}^{2}}+\Omega^{\prime}, \quad \Omega^{\prime}=v a_{o}^{2}
$$


In the case where we have BF unstable modes, we must have:

$$
\omega=\omega_{o}+\Omega=\omega_{o}+C_{g}\left(k-k_{o}\right)+i \mu \kappa^{2} \sqrt{I_{B F 2}^{2}-1}+v a_{o}^{2}, \quad \kappa=\sqrt{\left(k-k_{o}\right)^{2}-2 l^{2}}
$$

We can then see the real and imaginary parts of the frequency:

$$
\begin{aligned}
\omega= & \omega_{r}+i \omega_{i} \\
\omega_{r} & =\omega_{0}+C_{g}\left(k-k_{0}\right) \\
\omega_{i} & =\mu \kappa^{2} \sqrt{I_{B F 2}^{2}-1}
\end{aligned}
$$

This must give a term in the quasiperiodic Fourier series that looks like

$$
a e^{i \omega t}=a e^{i\left(\omega_{r}+i \omega_{i}\right) t}=a e^{\left( \pm \mu \kappa^{2} \sqrt{\left.I_{B F 2}^{2}-1\right) t}\right.} e^{i\left[\omega_{0}+C_{g}\left(k-k_{0}\right)\right] t}
$$

If we use the real part of the Fourier mode, we have an oscillatory wave:

$$
e^{i \omega t}=a e^{i\left[\omega_{0}+C_{g}\left(k-k_{0}\right)\right] t}
$$

The imaginary part of the Fourier mode will have the exponential growing (decaying) form

$$
a e^{i \omega t}=a e^{\left(\mu \kappa^{2} \sqrt{I_{B F 2}^{2}-1}\right) t}
$$

The directional behavior of (218) (and likewise (216)) and the 2+1 NLS equation have been discussed in depth in Section 4.

This waveform is exponentially increasing (or decreasing) with time, reflecting the BF instability. The expression (222) is quite surprising, because it says that there are exponentially growing and decaying modes of the $2+1$ NLS equation that are not the normal oscillatory ones: Their role is to give a modulationally unstable amplitude for the Stokes wave components in the nonlinear spectrum. Therefore, Stokes waves are not stable in deep water. Thus, the simple fact that we have exponential modes (rather than oscillatory ones) means we have identified an instability in the wave motion. We know there must be another similar mode in the neighborhood, phase locked to this one, coupled in such a way as to form a breather structure (see Section 4 for a discussion of the associated Riemann spectrum and their associated theta functions).

\section{Application to Analysis of SINTEF Wave Tank Data}

This section describes results from experiments conducted at Marintek in the early 2000s (Norwegian Marine Technology Research Institute), a company now known as the SINTEF Group, located in Trondheim, Norway. The technical details of the facility can be found on the Web site https://www.sintef.no/en/all-laboratories/towing-tanks/. The tank, at that time, was under the directorship of Dr. Carl Trygve Stansberg who in this capacity was crucial to the success of the experiments. He was also a participating scientist on the project. The tank is $260 \mathrm{~m}$ long and consists of two sections. The first section after the wave maker is $85 \mathrm{~m}$ long and has a width of $10.5 \mathrm{~m}$ and a depth of $10 \mathrm{~m}$. The second section is $175 \mathrm{~m}$ long, a width of $10.5 \mathrm{~m}$, and a depth of $5.6 \mathrm{~m}$. Various statistical analyses of the data have been conducted and published elsewhere (see Osborne [1] and cited references).

The scope of this Section is (1) to test the SINTEF data for the Benjamin-Feir (BF) instability and (2) determine the nonlinear spectrum of the data. We first seek to determine if the BF instability condition $k h>1.363$ holds. If so, we expect the data to be unstable and any initial modulation at the wave maker will grow exponentially. Therefore, the instability of the Stokes wave would be clear in these experiments. The BF condition implies $k>1.363 / h=0.136 \mathrm{~m}^{-1}$. Now the experiments discussed 
below have periods of $1.3,1.5 \mathrm{~s}$ and these correspond to $k h=23.82$ and $k h=17.88$ both of which are $>$ 1.363 , so the BF instability applies in both experiments, provided we assure that $I_{B F}>1$, which we have done. Our goal is to see what the data say about the physical behavior of the space/time dynamics of the instability in these two cases. According to Benjamin and Feir $[10,40]$, there should be an exponential growth of the amplitude of the modulation for unstable cases. They used linear instability theory to arrive at this conclusion, so that they were unable to describe the long-time behavior of a BF unstable wave train. While this behavior is theoretically well known to manifest breathers during their long-time evolution (Osborne [1] Chapters 12, 18, 24, 29), it should be clear that there is the necessity of demonstrating that breathers should robustly occur in experimental data. The SINTEF data discussed below were among the first experimental demonstration that breather formation is a generic feature of the long-time evolution of deep-water gravity waves.

Figure 24 shows the results of the first experiment (see Osborne [1] Chapter 29 and references), which consisted of a modulated Stokes wave, generated by the split paddle in the long wave flume. As indicated in the figure caption of Figure 24, we generated a sine wave with period of $1.3 \mathrm{sec}$, which was slightly modulated with a period of $19.5 \mathrm{~s}$ at the wave maker. There are $7.6 \mathrm{such}$ modulations in the $150 \mathrm{~s}$ time series, measured at 13 probes from $10 \mathrm{~m}$ to $85 \mathrm{~m}$ from the wave maker. Each probe is numbered on the right-hand side of the graph from the lower-most time series (probe 1 at $10 \mathrm{~m}$ ) out to the upper-most time series (last probe 13 at $85 \mathrm{~m}$ ). The modulation has grown considerably already at Probe 1. We see a train of modulations, each occurring at $19.5 \mathrm{~s}$ intervals. Subsequent probes show that the modulation not only continues its growth, but also begins the BF self-focusing process that leads to a train of localized wave packets by about $70 \mathrm{~m}$ from the wave maker. These wave packets are actual breathers that occur periodically at the $19.5 \mathrm{~s}$ interval of the initial modulation. If we continue the experiment for longer times, the breathers return to their initial small amplitude conditions, then regrow up to the breather packet state again, repeating the so-called Fermi-Pasta-Ulam recurrence cycle. Thus, for a single modulated Stokes wave, we see the initial growth of the small amplitude modulations for early times, the manifestation of breathers over longer time scales and the FPU recurrence over even longer time scales. FPU recurrence breaks down at sufficiently long time periods (Osborne [1]). The breather trains can be physically described by Equations (24.22)-(24.29) in Osborne [1] (see Chapters 24 and 29).

Figure 25 shows the results of another experiment (again further details are given in Osborne [1] and cited references) with random wave trains, generated by SINTEF software, for which the JONSWAP power spectrum with random Fourier phases was used to drive the wave maker. Line segments are used to help the eye follow the evolution of wave packets in the measured data.

At the first probe at $10 \mathrm{~m}$ from the wave maker (the lowest time series in Figure 25), we see considerable packet structure, but the packets are relatively low and broad, primarily because the JONSWAP spectrum driving the wave maker was high and narrow. We compare this signal to the signal measured at probe 8 of Figure 25 that is $70 \mathrm{~m}$ from the wave maker, regraphed in Figure 26. In this latter time series, we see packets that are quite narrow and considerably higher than those at probe 1. One packet has a central wave height of $32.53 \mathrm{~cm}$ whose amplitude is over 5 standard deviations. Another packet has a height of $31.07 \mathrm{~cm}$ with amplitude of 4.8 standard deviations. A third packet has a wave height of $23.75 \mathrm{~cm}$, which occurs at about three standard deviations.

In Figure 25 the behavior of the wave packets at probe 1, only $10 \mathrm{~m}$ from the wave maker, is seen to be relatively broad and low, and is nearly Gaussian in its statistical behavior. The behavior at probe 8 is highly non-Gaussian in the amplitudes and non-Rayleigh in the wave heights. The important point is that by ensuring that the modulational parameter was large for these random wave experiments, we were able to elicit the BF instability in random wave trains. Indeed, the single most important point is that we enhanced the constant $\gamma$ in the JONSWAP spectrum in order to add the non-equilibrium peak on the spectrum, thus making the spectrum high and narrow. The random wave trains, Gaussian and linear by construction at the paddle, were soon influenced by the modulational instability and the 
large narrow packets were in some sense "fissioned" from the long and low initial wave packets as initial conditions.

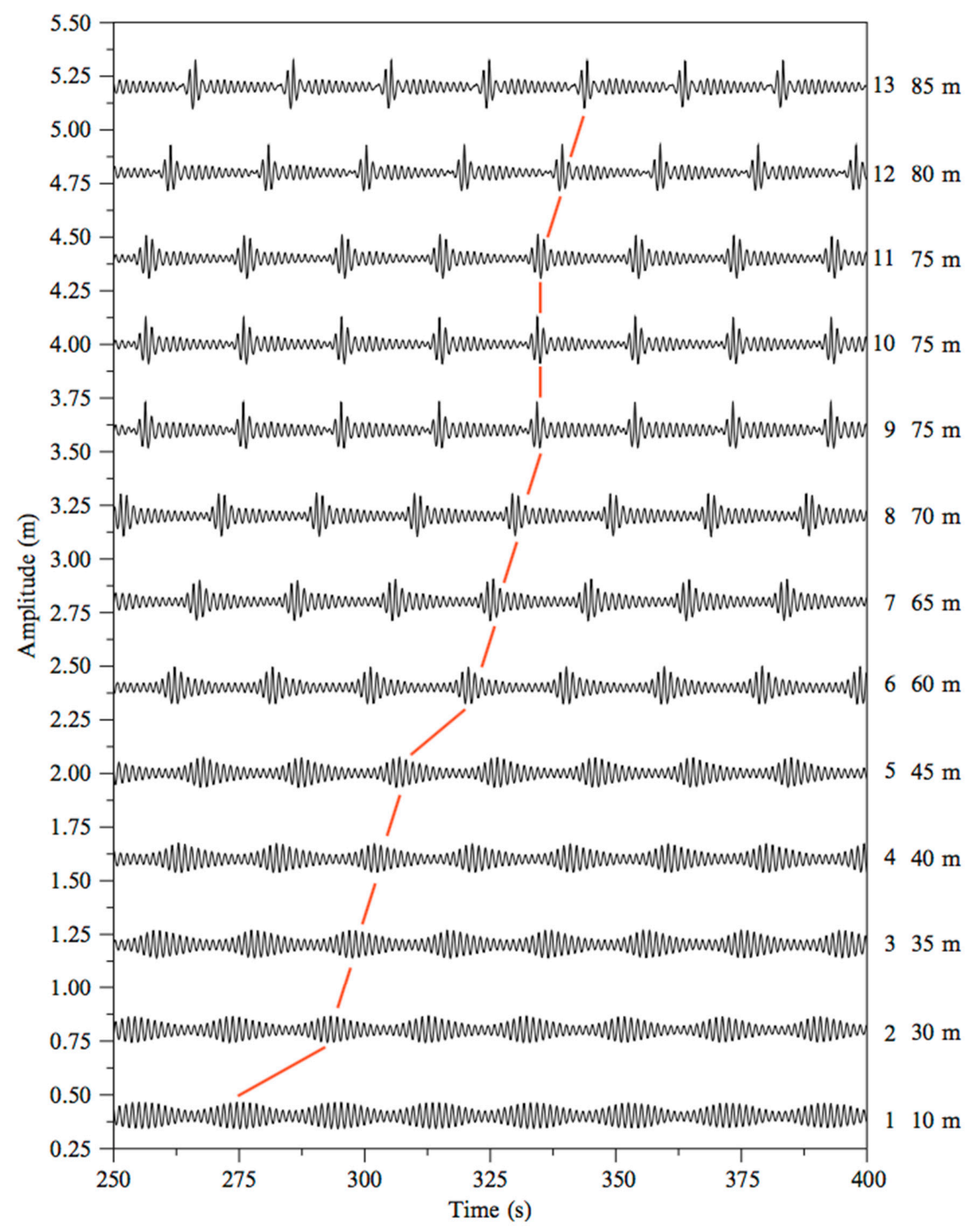

Figure 24. A $150 \mathrm{~s}$ section of an experiment conducted at SINTEF in the long wave flume. Here a sine wave (carrier wave period of $1.3 \mathrm{sec}$ ) is given a small-amplitude modulation (period $19.5 \mathrm{sec}$ ) at the wave maker at $0 \mathrm{~m}$. Here 13 wave probes are shown ordered from the bottom to the top of the figure: See probe numbers and distances from wave maker on the right. About eight carrier modulations are shown. From the figure, we see that the sine wave created by the wave maker is modulationally unstable because the modulation has grown as it propagates from the wave maker to the first probe at $10 \mathrm{~m}$. The modulation continues to grow and focus as the wave train propagates down the tank to a distance of $85 \mathrm{~m}$. The smooth modulations near the wave maker are transformed during their propagation down the tank: Beyond about $70 \mathrm{~m}$, the wave amplitudes consists of seven localized packets that we call breathers. This is one of the best experimental evidences of the long-time evolution of the Benjamin-Feir instability. 


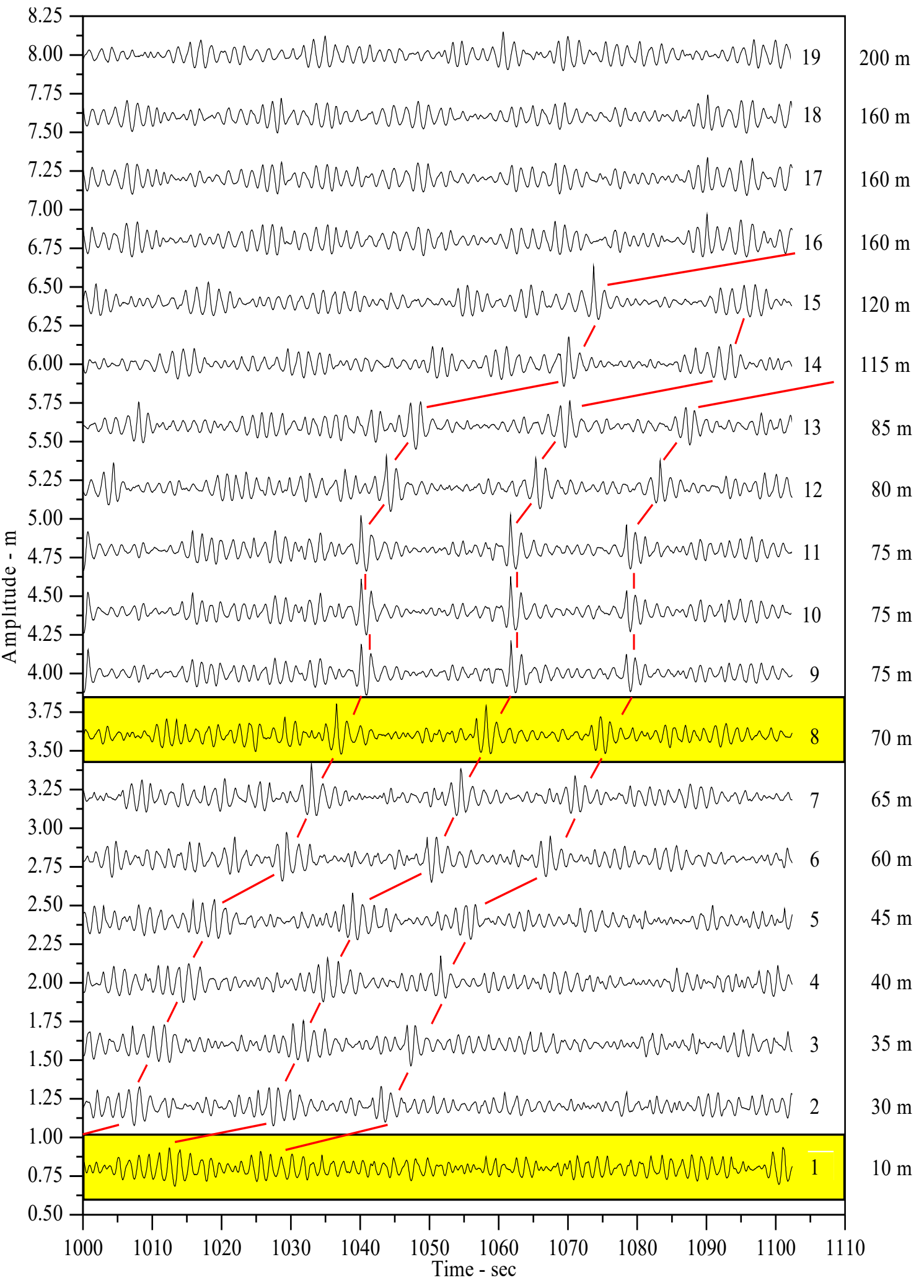

Figure 25. A $100 \mathrm{~s}$ section of a random wave experiment conducted at SINTEF. We used the value $\gamma=6$ for the JONSWAP power spectrum. A total of 19 probes were placed along the tank, see probe numbers and distances down the right-hand side of the figure. The probe at $10 \mathrm{~m}$ has long and low packets corresponding to a high and narrow JONSWAP spectrum. Self-focusing occurs at later probes, leading to the formation of breather packets. 


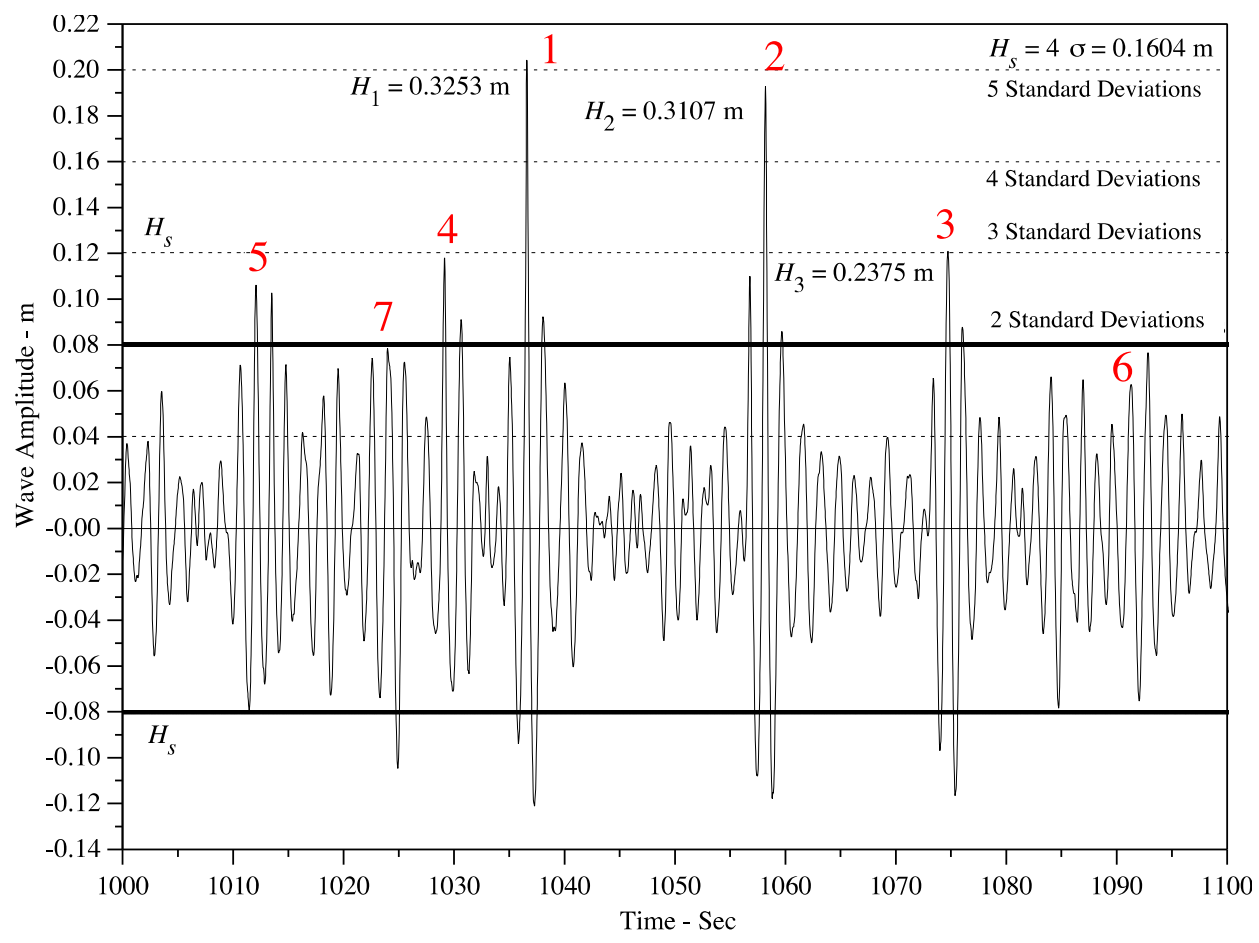

Figure 26. A 4096-point time series from probe 8 at $70 \mathrm{~m}$ from the wave maker in the experiments of Figure 25. Three extreme waves have amplitudes that are greater than three standard deviations. The largest packets (associated with their NLFA components, see Figure 20) are indicated by red numbers. The largest packet is greater than twice the significant wave height.

In Figure 27, we show the results of the nonlinear Fourier analysis on the time series of Figure 26. Note that the horizontal frequency axis is centered at the peak of the spectrum where the frequency is taken to be zero. It is now easy to interpret this spectrum in terms of Figure 1 and the related discussion. Note that the small amplitude components in the left- and right-hand tails of the spectrum are simple sine waves whose amplitudes are indicated by a cross (a single simple eigenvalue) and that are shown connected to the frequency axis by a vertical line, a "spine." Stokes wave components are similarly indicated by a cross, but their spines (see the definition and numerical information in Osborne [1] and Osborne et al. [5]) are not typically straight lines, but instead they are distorted or curved, while still connecting to the frequency axis. The other type of spectral component consists of unstable wave packets or breathers. These latter consist of two points of simple spectrum (denoted by crosses) connected by a spine without touching the frequency axis. The low amplitude breathers are easy to see as the two points of simple spectrum are well separated and connected by a spine, often seen as a "loop." Higher amplitude breathers have the two points of spectrum very close to each other (a mathematician would call them "nearly homoclinic"), so much so that one cannot graph the spine, as it is too short to see.

There are 14 unstable modes or breathers in the spectrum of Figure 27, the larger of which are candidates for extreme waves at some point during their nonlinear evolution. The largest breathers are numbered in red on the time series in Figure 26, corresponding to each numbered large breather component in the spectrum of Figure 27, so that we can see which breather is associated with each spectral component.

Breathers are a kind of "coherent structure" born of the nonlinearities in the water wave equations. In two-dimensional turbulence, the coherent structures are elliptic vortices, while in water waves the coherent structures are breather packets: Mathematically, they are hyperbolic vortices. The fact that there are so many breathers in the SINTEF data suggests that a kind of breather turbulence is dominating 
the water wave dynamics (see Osborne et al. [5] for a study of breather turbulence in Currituck Sound, also discussed below).

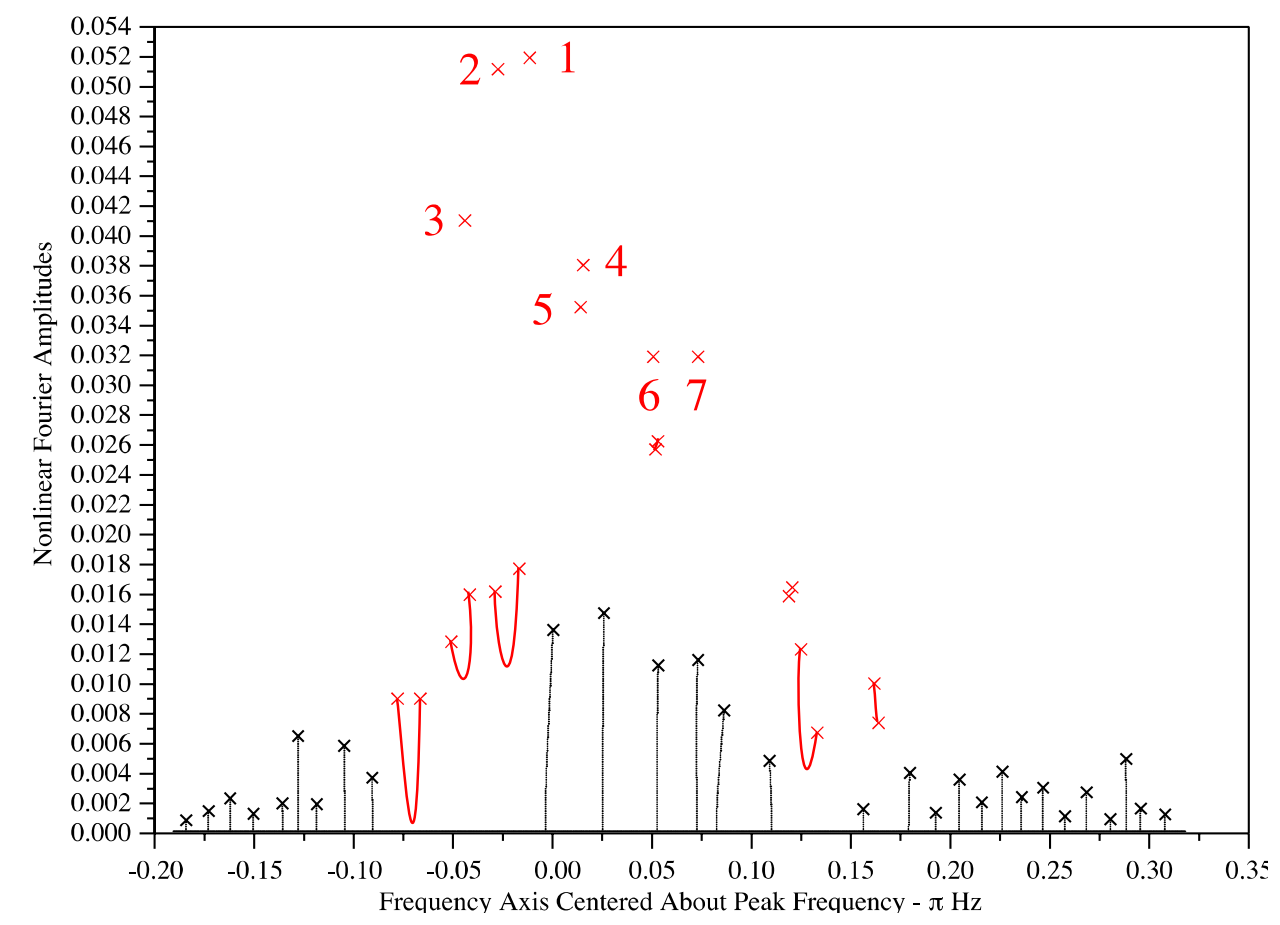

Figure 27. The NLFA spectrum for the time series in Figure 26. Sine wave and Stokes wave components are shown by black crosses connected to the frequency axis by a "spine." See discussion with regard to Figure 1. Breathers are colored red and consist of two points of simple spectrum (red crosses) connected by a spine (a red line). When the two points of simple spectrum are too close, the spine is not visible and only a single cross is seen. This happens for the largest breathers near the peak of the spectrum. The numbers near the largest breathers coincide with those in the measured time series in Figure 26. This emphasizes the one to one nature of the nonlinear spectral components of breathers (this figure) and the breathers themselves (Figure 26) in the time domain.

\section{Application to Analysis of Wave Data in Currituck Sound}

Figures 28-30 show some of the results of the analysis of data taken in Currituck Sound. Details can be found in Osborne et al. and cited references [5]. Here only the basic results are shown in order to illustrate the NLFA method. A measured time series from Currituck Sound (band pass filtered to remove the low frequency component, see details of the analysis in Osborne et al. [5]) is shown in Figure 28. One can see the strong Stokes wave component in the figure: This is seen by the substantial up/down asymmetry in the data, which has been removed in Figure 29, so that the time series is now up/down symmetric, a requirement of the $1+1$ NLS equation. The real modulation of the data is shown as a red line that coincides with the peaks of the waves.

The NLFA spectrum of the Currituck Sound data is shown in Figure 30. We see that the spectrum is dominated by breather states. Virtually all the red dots in the upper part of the spectrum are breathers whose two eigenvalues are so close together that the spines are too short to be visible by eye. The ten largest breathers are numbered in red: These are the packets seen in the time series of Figure 29, also numbered in red.

The smaller spectral points, near the frequency axis, have spines that drop down to the frequency axis: These are sine and Stokes waves. Other components near the frequency axis consist of two points of a simple spectrum connected by a spine. These are very small "baby" breathers. The largest three breathers have maximum heights greater than $2.2 \mathrm{H}_{s}$ and their central waves are therefore classified as rogue waves. There are over 200 large breathers in the measured wave train of Figure 29. Indeed, 
the density of these breathers is so great that the wave field can be thought of as breather turbulence. This is a shocking result as it says that the theoretical paradigm of weakly interacting sine waves is no longer tenable for describing ocean waves. Only a wave theory that includes coherent structures such as Stokes waves and breathers can adequately describe the breather structure of a time series like that in Figure 28. The paper by Osborne et al. [5] thus describes the birth of a new field, which they referred to as coherent wave turbulence or breather turbulence. The power of NLFA is clear in this example.

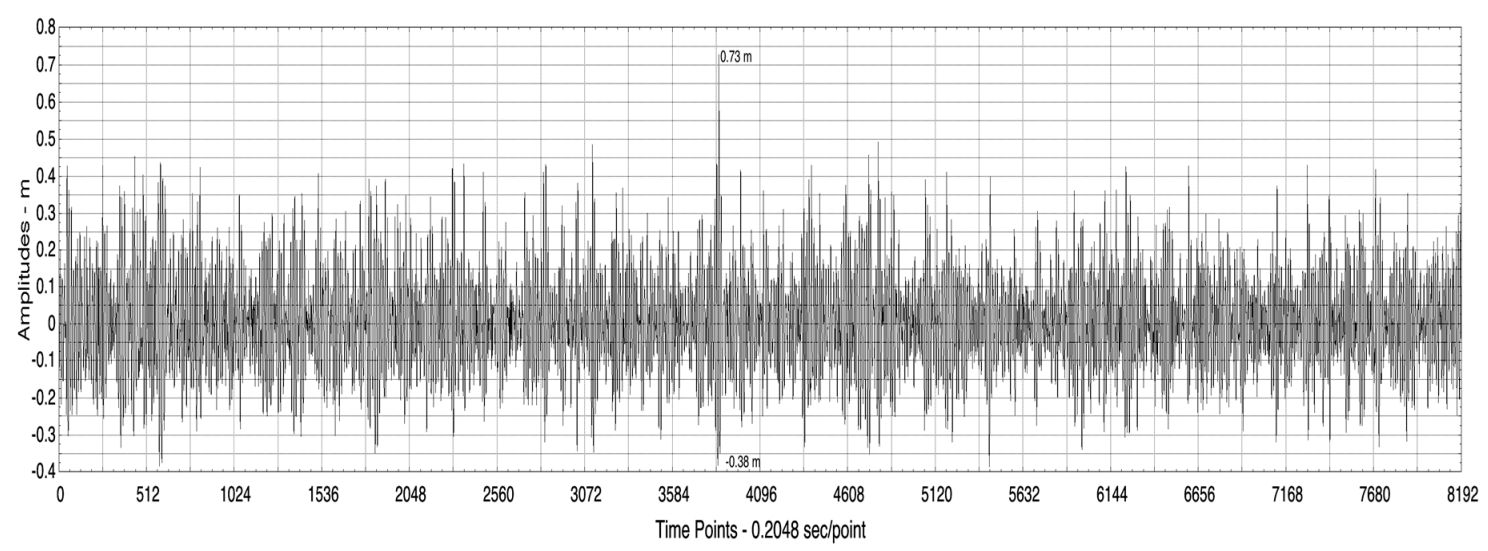

Figure 28. Time series of the Currituck Sound data after the removal of the low frequency part of the spectrum. This series is consistent with the known physics of the $1+1$ NLS equation. The standard deviation of the filtered time series is $13.45 \mathrm{~cm}$ and significant wave height is $53.8 \mathrm{~cm}$. The largest wave height is $111 \mathrm{~cm}$, corresponding to $2.06 \mathrm{H}_{s}$.

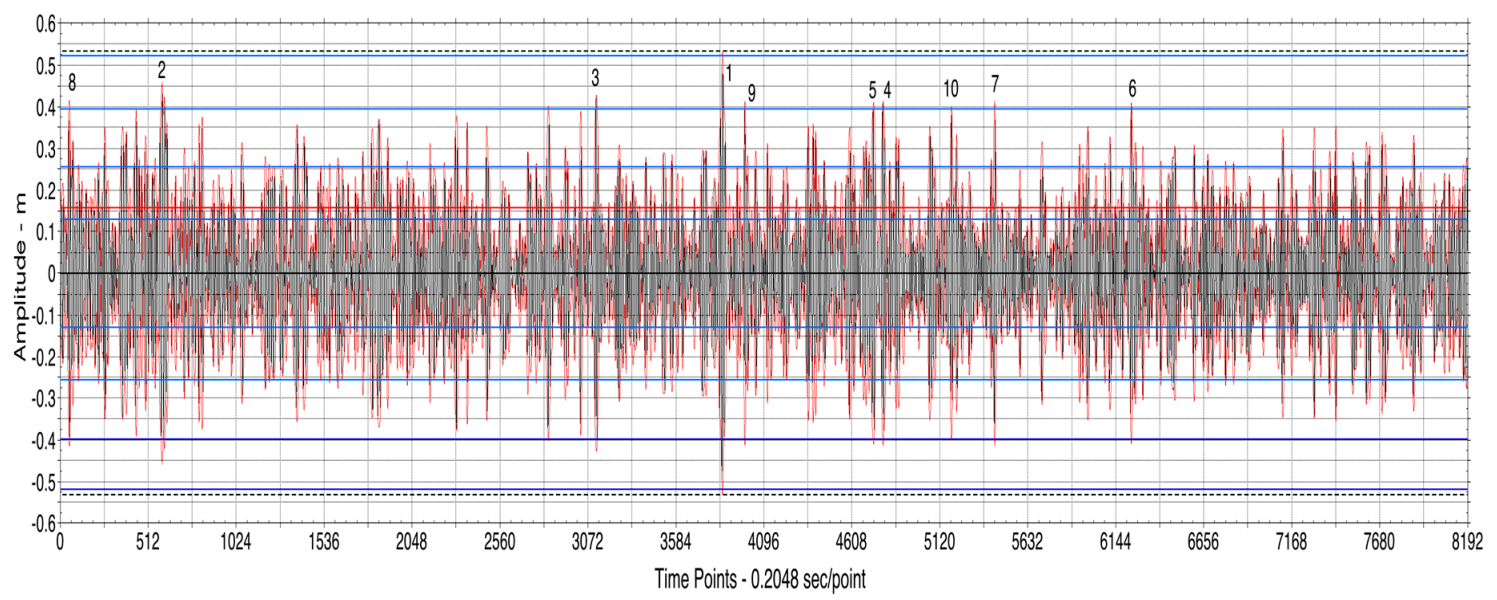

Figure 29. The measured time series of Figure 28 has been processed by the inverse nonlinear Stokes operator (Osborne et al. [5]) to obtain the present time series. As a consequence, the time series is up/down symmetric, the Stokes wave contribution has been removed, a condition consistent with further analysis using NLFA for the 1+1 NLS equation (see Figure 30). Also shown (red) is the modulational envelope $A(0, t)=\left[\eta(0, t)^{2}+\vec{\eta}^{2}\right]^{1 / 2}$ found by the Hilbert transform of the above time series for which the carrier amplitude is $a_{0}=16 \mathrm{~cm}$. The significant wave height of this time series is $52.0 \mathrm{~cm}$. The height of the largest wave (labeled 1) is $2.04 H_{s}$. The numbers labeling the largest ten packets coincide with the largest packets in the NLFA spectrum shown in Figure 30. The largest three packets have central waves that are bigger than $2.2 H_{S}$ and are therefore classifiable as rogue waves. 


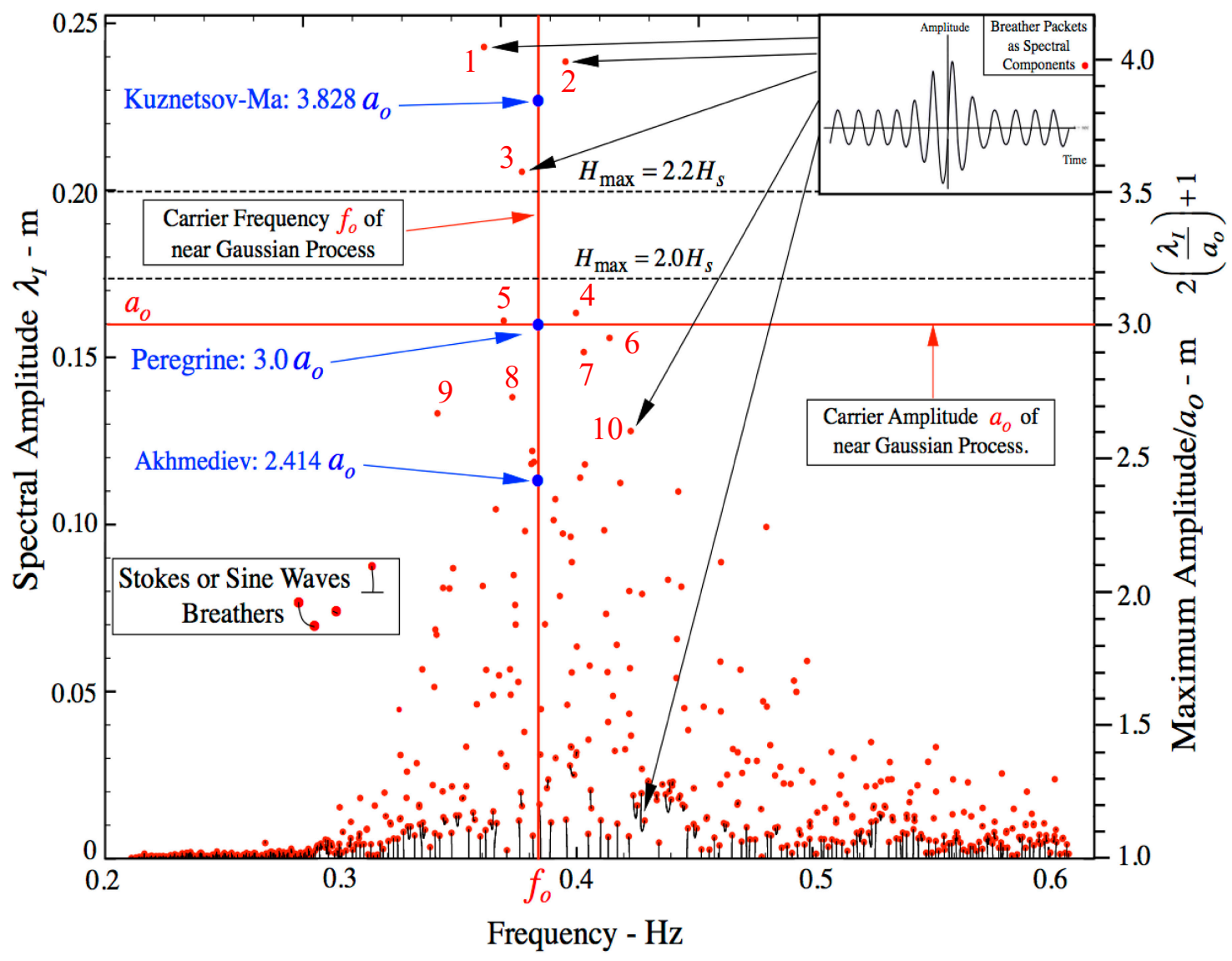

Figure 30. The nonlinear Fourier (NLFA) spectrum for the complex modulation function obtained from the time series in Figure 29. Note that there are two vertical axes. The one on the left is the actual eigenvalue obtained in the computation of the NLFT spectrum and has units of meters. The right-hand vertical axis is dimensionless, and it is effectively the maximum amplitude of a breather normalized by the carrier amplitude. This means that the actual carrier amplitude $a_{0}=16 \mathrm{~cm}$ (left vertical axis, see the horizontal red line $a_{0}$ and see horizontal red line in Figure 29) and the normalized amplitude is 3 (see the right-hand vertical axis). The actual positions of the three theoretical breathers most often cited (Akhmediev, Peregrine, and Ma-Kuznetsov) are shown in blue. Spectral data points above $2.2 \mathrm{H}_{S}$ (there are three of them) are considered to be rogue waves. The red numbers labeling the ten largest breather packets coincide with the ten largest packets of the time series in Figure 29.

\section{Application to Radar Data Assimilation and Modeling, and Real-Time Prediction of Ocean Waves}

This section briefly describes ongoing work for the U. S. Navy on the assimilation of radar data of ocean surface waves and the subsequent prediction of waves for the near future in real-time situations onboard naval vessels. The radar data discussed here were measured by Sutherland and Melville [61-63] and provided to the author by Linwood Vincent.

A typical radar scan is shown in Figure 31. The conversion to actual wave heights is shown in Figure 32 where the methods of Cheng and Chien [64] and Lund et al. [65] are applicable. Measurements of this type and the subsequent data conversion can be made in real time aboard a ship with radar and onboard computing capability. 


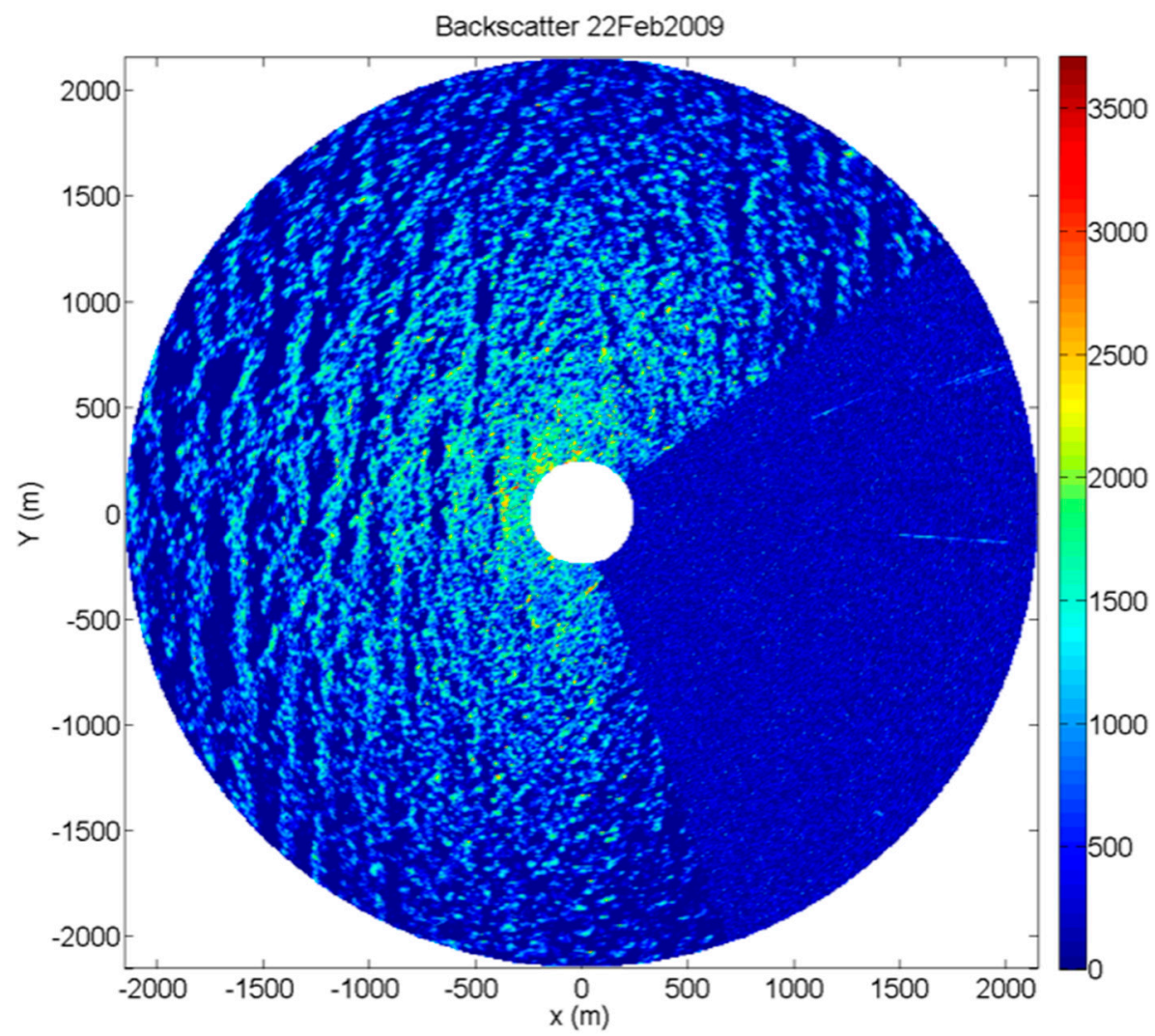

Figure 31. Radar measurements reported by Sutherland and Melville [62,63].

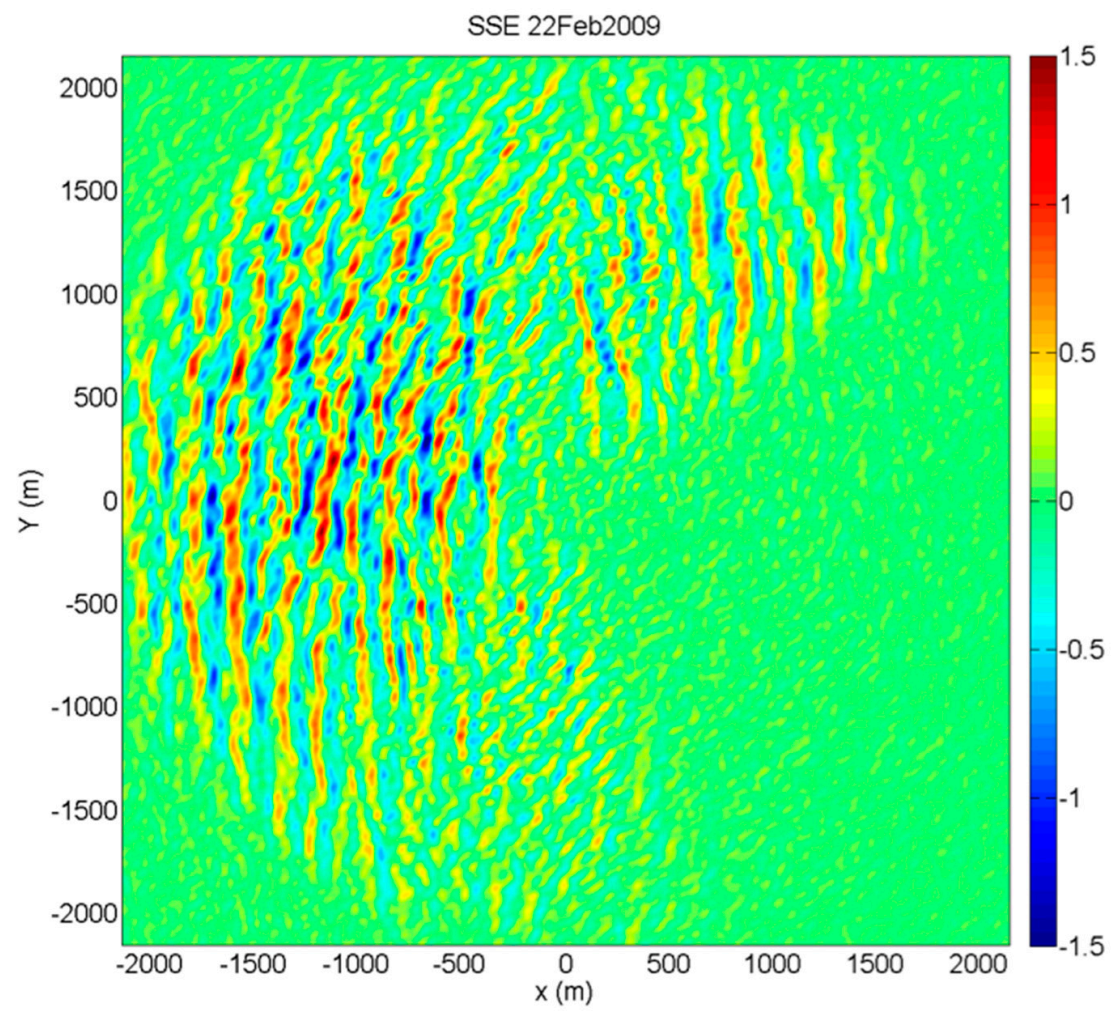

Figure 32. Conversion of radar measurements of Figure 19 to surface waves, see, for example, [Cheng and Chien, 17] [Lund et al., 45]. 
The goal of this program is to provide a real-time, onboard modeling capability that allows for radar measurements to be input to the software such that the data are assimilated to determine the Riemann spectrum (Riemann matrix, frequencies, and phases). The two-dimensional generalization of the method in the flowchart of Figure 15 is used for this purpose. Then, the wave field in the near future is computed by the flow chart given in Figure 16, and the results are shown in Figure 33. The reason that the model presented here is very fast is because quasiperiodic Fourier series (see (65)) can be reduced to an ordinary 2D Fourier series (see (66) and related discussion in Section 5): This allows use of a 2D FFT algorithm to compute the wave motion after the pre calculation of the quasiperiodic Fourier series for the coefficients (this was referred to as a "preprocessor" by Osborne [1]). Both of the theta functions corresponding to the two sets of phases are computed in a single subroutine. Thus, the method of Riemann theta functions used here is quite fast, being only about $20 \%$ slower than the linear model ordinarily computed by the two-dimensional FFT (see also Osborne [1]) for additional mathematical and numerical details for these kinds of models).

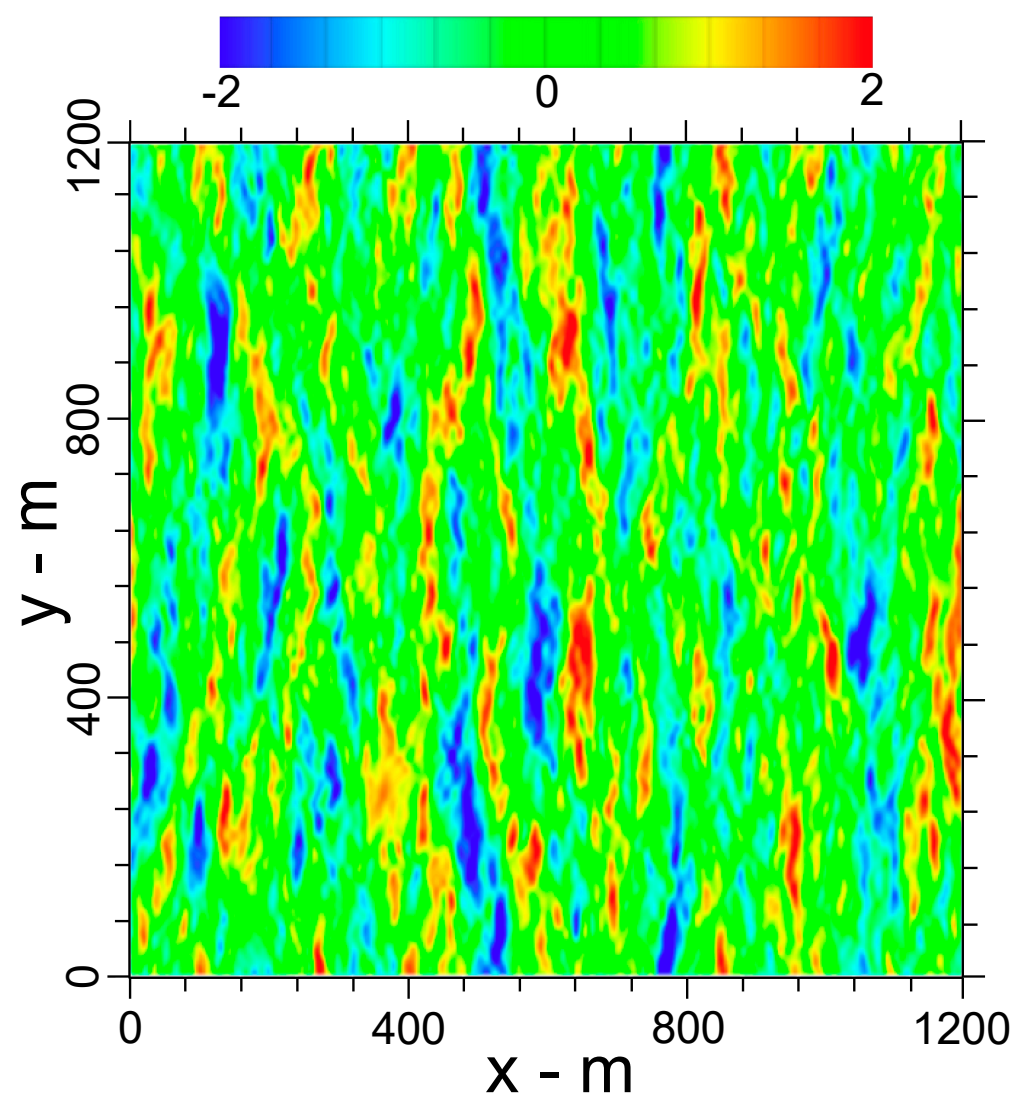

Figure 33. Wave simulation using input radar data and the algorithm in the flow chart in Figure 16 extended to two dimensions. See Osborne [1] for additional numerical methods.

\section{Summary and Discussion}

This paper has focused on the application of methods of nonlinear Fourier analysis through the use of the application of the inverse scattering transform for determining asymptotic solutions for the hierarchy of nonlinear Schrödinger equations with an arbitrary potential. The method, as outlined herein, gives a wave model for the surface wave elevation for wave motion in two dimensions to arbitrarily high order, although herein we have focused primarily on asymptotic solutions of the $2+1$ NLS equation. Extension to arbitrary orders is carried out by improving estimates of the parameters in the Riemann spectrum by theoretical means as discussed here. Another issue not discussed but which has important future applications relates to placing slower time dependence in the NLFA 
coefficients (an approach already suggested by Poincaré in his classical work of 1892). The real-time estimation of the Riemann spectrum (period matrix, frequencies and phases) provides a fast method for assimilating radar data and for predicting the future of ocean waves in real time.

Future applications of NLFA in a number of areas of offshore engineering are large in number. Understanding the details of platform and offshore structure design in the presence of long-lived, extreme amplitude breather packets is an area of importance. Platform dynamics and ringing are other areas of research that need exploration (Gudmestad [66]). Understanding wave forces due to the Morison equation using nonlinear Fourier methods is also an open area of research and could have an impact on characterization of ringing dynamics. Static ship design and dynamics under nonlinear wave forces computed by the NLFA approach is another area to explore. The analysis and reanalysis of laboratory and ocean wave data using NLFA are a frontier area of research. New searches for innovative ways to analyze data in novel situations will provide creative approaches for the study of myriad areas of research in ocean engineering.

It is worthwhile pointing out that this is the first effort to use quasiperiodic Fourier series for the modelling of ocean waves and for 1D space/time series analysis and for 2D spatial field analysis of surface wave data. This has not been an easy task because of the lack of physical and mathematical understanding of the quasiperiodic Fourier series in theoretical and physical methods for the solutions of nonlinear wave equations. This has required modern understanding of the inverse scattering method for periodic/quasiperiodic boundary conditions. A recent leap has been to discover how the solutions of these equations can be written in terms of quasiperiodic Fourier series. Historically, a long road has been followed by dozens of investigators over the two centuries since Fourier's paper in 1922. The present paper is unique because it has developed the new numerical methods to determine how to exploit the wonderful mathematics of harmonic analysis, which has been available for over a century, but only now is being fully utilized. Continued development of this area of research over the next few years is anticipated. Applications to many fields, including internal waves are expected. Additional applications to solid state physics, nonlinear optics, quantum mechanics, and other fields are foreseen.

Funding: We acknowledge Tom Drake of the Office of Naval Research for funding the present research (N00014-16-C-3001). We greatly appreciate ONR funding over the past decades. This research was partially funded by SINTEF Ocean, Norway in the frame of the Workshop "Future Paths and Needs in Wave Modelling." We also acknowledge members of the offshore and shipping industries that have funded this work.

Acknowledgments: The author thanks Alex Babanian, Elzbieta Bittner-Gregerson, Odin Gramstad, Sverre Haver, Sonia Ponce de León, Carl Trygve Stansberg, Alessandro Toffoli, Karlsten Trulsen, and Ian Young for helpful comments and constructive criticisms over the many years of this research.

Conflicts of Interest: The author declares no conflicts of interest.

\section{Appendix A. What Is Fourier or Harmonic Analysis?}

This discussion of Fourier analysis is divided into three different ways of obtaining Fourier series for describing and modeling linear and nonlinear wave motion. The three types of Fourier analysis are ordered in terms of increasing generality and complexity. The first type is the standard periodic Fourier series, which is ordinarily applied numerically as the fast Fourier transform in science and engineering. The second type is the quasiperiodic Fourier series that, up to now, has had little application in oceanography and ocean engineering. The method as applied here derives from the inverse scattering method for solving nonlinear wave equations with periodic/quasiperiodic boundary conditions. The third type is the almost periodic Fourier series, the most beautifully delicate and mathematically sophisticated of the methods, which has been used mainly as a theoretical tool for the study of solutions of nonlinear differential and wave equations. The primary tool applied herein is the quasiperiodic Fourier series, which is used in applications to the analysis of surface wave data in ocean and laboratory data. 


\section{1:. Standard Periodic Fourier Analysis}

The periodic Fourier series of a function $u(x)$ (Fourier [67], Zygmund [68]) is given by

$$
u(x)=\sum_{n=-\infty}^{\infty} u_{n} e^{i k_{n} x}, \quad k_{n}=\frac{2 \pi n}{L}
$$

We have periodic motion for the function $u(x)=u(x+L)$ as well as for the basis $e^{i k_{n} x}$ with period $L$. These are the pure periodic functions in classical analysis. The wavenumbers are non-denumerable (there are infinitely many of them) and commensurable (equally spaced).

Periodic Fourier series solve linear wave equations:

$$
u(x)=\sum_{n=-\infty}^{\infty} u_{n} e^{i k_{n} x-i \omega_{n} t+i \phi_{n}}, \quad k_{n}=\frac{2 \pi n}{L}, \quad \omega_{n}=\omega_{n}\left(k_{n}\right)
$$

Here $\omega_{n}=\omega_{n}\left(k_{n}\right)$ is the nonlinear dispersion relation. The wavenumbers are non-denumerable (there are infinitely many of them) and commensurable (equally spaced). The frequencies $\omega_{n}=\omega_{n}\left(k_{n}\right)$ instead are also non-denumerable (there are infinitely many of them) and incommensurable (not equally spaced because of the form of the nonlinear dispersion relation for water waves).

If one writes the Fourier series (A1) as a discrete Fourier series, it then becomes amenable to numerical computation either directly or with the fast Fourier transform (FFT). Standard periodic Fourier series are very familiar to scientists and engineers who use the FFT to numerically compute wave motion.

\section{2:. Quasiperiodic Fourier Series}

The quasiperiodic Fourier series has the form (Bohl [22]):

$$
u(x)=\sum_{\mathbf{n} \in \mathbb{Z}^{N}} u_{\mathbf{n}} e^{i \mathbf{n} \cdot \mathbf{k} x}
$$

The fact that we have a quasiperiodic function means that we can write symbolically, $u(x) \approx u(x+L)$, which means only approximate equality, not the perfect equality in Section 1 . Both the summation vector $\mathbf{n}=\left(n_{1}, n_{2} \ldots n_{N}\right)$ and wavenumber vector $\mathbf{k}=\left(k_{1}, k_{2} \ldots k_{N}\right)$ have $N$ denumerable components. Therefore, the vector of wavenumbers is denumerable and incommensurable (unequally spaced). Quasiperiodicity in the space variable $x$ happens because the wavenumber components are incommensurable. The compact summation notation above can be written in expanded form in the following way:

$$
u(x)=\sum_{\mathbf{n} \in \mathbb{Z}^{N}} u_{\mathbf{n}} e^{i \mathbf{n} \cdot \mathbf{k} x}=\sum_{n_{1}=-\infty}^{\infty} \sum_{n_{2}=-\infty}^{\infty} \ldots \sum_{n_{N}=-\infty}^{\infty} u_{n_{1} n_{2} \ldots n_{N}} e^{i\left(n_{1} k_{1}+n_{2} k_{2}+\ldots+n_{N} k_{N}\right) x}
$$

Thus, we see that there are $N$ nested sums in a quasiperiodic Fourier series. This is a huge departure from standard Fourier series (A1) and influences the work of the scientist or engineer in at least two ways: (1) His conception about the physics of nonlinear waves (which are now nonlinearly interacting with one another through the nested summations) must be substantially improved and (2) his development of numerical algorithms (which now can be quite onerous) requires ever new and improved approaches. Indeed, one of the main reasons for the book by Osborne [1] is to provide advances in physical understanding of integrable nonlinear wave motion for periodic/quasiperiodic boundary conditions and to improve the computational speed of the consequent quasiperiodic Fourier series. A crucial step in this understanding is the use of Riemann theta functions, themselves quasiperiodic Fourier series, because their numerical computation can be accelerated by many orders 
of magnitude (Osborne, Chapter 22 [1]) and because solutions of integrable wave equations can be constructed directly from theta functions.

Consequently, one can determine quasiperiodic Fourier series of nonlinear wave equations that are integrable by the inverse scattering method (KdV (Osborne [2])), KP (Osborne [4]), NLS (Osborne [3])). This is done by writing the solution of such an equation in the following form:

$$
u(x, t)=\sum_{\mathbf{n} \in \mathbb{Z}^{N}} u_{\mathbf{n}} e^{i \mathbf{n} \cdot \mathbf{k} x-i \mathbf{n} \cdot \boldsymbol{\omega} t+i \mathbf{n} \cdot \boldsymbol{\phi}}
$$

Generally, the wavenumbers, frequencies, and phases are all incommensurable (unequally spaced) and denumerable (vectors of length $N$, the so-called genus) in the solutions of nonlinear integrable wave equations. Of course, determination of the Fourier parameters for a particular Cauchy initial condition is key to the success of the method. To solve nonlinear integrable wave equations by a linear superposition of sine waves (A5) may seem like a miracle, if not impossible, but it is the consequence of the generality of the mathematics of quasiperiodic Fourier series and the integrability of certain nonlinear wave equations of interest for the study of water waves.

Quasiperiodic Fourier series have been used since 1860 in the study of planetary motion (Delaunay [8]) and in the study of nonlinear ordinary differential equations with small degrees of freedom. The fact that there are $N$ denumerable degrees of freedom in a quasiperiodic Fourier series means that they are useful for describing space/time series of $N$ points. At this point, such series are no longer assumed to be periodic, but are instead taken to be quasiperiodic.

\section{3:. $\quad$ Almost Periodic Fourier Series}

The almost periodic Fourier series has the form (see H. Bohr [23], A. S. Besicovitch [24], S. Bochner [25,26], B. Jessen [27], Paley and Weiner [39], and C. Corduneanu [7]):

$$
u(x)=\sum_{n=-\infty}^{\infty} u_{n} e^{i k_{n} x}, \quad k_{n} \neq \frac{2 \pi n}{L}
$$

where the $k_{n}$ are incommensurable and non-denumerable. We thus have an infinite number of wavenumbers in an almost periodic Fourier series. We therefore have solutions of integrable wave equations that have the form:

$$
u(x)=\sum_{n=-\infty}^{\infty} u_{n} e^{i k_{n} x-i \omega_{n} t+i \phi_{n}}, \quad k_{n} \neq \frac{2 \pi n}{L}, \quad \omega_{n}=\omega_{n}\left(k_{n}\right)
$$

Notice that the wavenumbers are generally incommensurable, and so too are the frequencies. Part of the science, art, and mathematics of almost periodic Fourier series is to find these parameters to form the solution of a particular nonlinear wave equation. The book by Corduneanu [7] provides an excellent overview of this approach. As seen in the body of this paper, the quasiperiodic Fourier series for integrable wave equations can, for many practical purposes, be written as almost periodic Fourier series, leading to the conclusion that nonlinear wave motions can once again be represented as the linear superposition of sine waves.

A table of the Fourier Series used in this paper is given below:

Table A1. Characteristics of the Different Methods of Fourier Analysis.

\begin{tabular}{clc}
\hline Fourier Method & \multicolumn{1}{c}{ Wavenumber/Frequency } & Number of Spectral Components \\
\hline Periodic Fourier Series & Commensurable/Incommensurable & Non-denumerable \\
\hline Quasiperiodic Fourier Series & Incommensurable/Incommensurable & Denumerable \\
\hline Almost Periodic Fourier Series & Incommensurable/Incommensurable & Non-denumerable \\
\hline
\end{tabular}


From top to bottom in this table the Fourier series are in order of increasing mathematical difficulty and generality. The least general are the periodic Fourier series and the most general are the almost periodic Fourier series. The quasiperiodic Fourier series lie in between these two.

\section{References}

1. Osborne, A.R. Nonlinear Ocean Waves and the Inverse Scattering Transform; Academic Press: Boston, MA, USA, 2010; 976p.

2. Osborne, A.R. Nonlinear Fourier methods for ocean waves. Procedia IUTAM 2018, 26, 112-123. [CrossRef]

3. Osborne, A.R. Breather turbulence: Exact spectral and stochastic solutions of the nonlinear Schrödinger equation. Fluids 2019, 4, 72. [CrossRef]

4. Osborne, A.R. Theory of Nonlinear Fourier Analysis: The Construction of Quasiperiodic Fourier Series for Nonlinear Wave Motion. In Proceedings of the ASME 2020 39th International Conference on Ocean, Offshore and Arctic Engineering OMAE2020-8536, Ft Lauderdale, FL, USA, 28 June-3 July 2020.

5. Osborne, A.R.; Resio, D.T.; Costa, A.; Ponce de León, S.; Chrivì, E. Highly nonlinear wind waves in Currituck Sound: Dense breather turbulence in random ocean waves. Ocean Dyn. 2018, 69, 187-219. [CrossRef]

6. Samoilenko, A.M. Elements of the Mathematical Theory of Multi-Frequency Oscillations; Kluwer Academic Publishers: Dordrecht, The Netherlands, 1991.

7. Corduneanu, C. Almost Periodic Oscillations and Waves; Springer: New York, NY, USA, 2009.

8. Delaunay, C. Théorie du Mouvement de la Lune; T. T. Malet-Bachelier: Paris, France, 1860.

9. Poincaré, H. Periodic and Asymptotic Solutions; American Institute of Physics: University Park, MD, USA, 1993; Volume 1.

10. Poincaré, H. Approximations by Series; American Institute of Physics: University Park, MD, USA, 1993 ; Volume 2.

11. Poincaré, H. Integral Invariants and Asymptotic Properties of Certain Solutions; American Institute of Physics: University Park, MD, USA, 1993; Volume 3.

12. Kolmogorov, A.N. On the Preservation of Conditionally Periodic Motions Under a Small Change of the Hamiltonian. Dokl. Akad. Nauk SSSR 1954, 98, 527-530.

13. Arnol'd, V.I. Small Denominators I: On the Mappings of a Circle onto Itself. Izv. Akad. Nauk Ser. Mat. 1961, 25, 21-86.

14. Arnol'd, V.I. Small Denominators: A Proof of A.N. Kolmogorov's Theorem on Conservation of Conditionally Periodic Motions Under a Small Change of the Hamiltonian. Uspekhi Mat. Nauk 1963, 18, 13-40.

15. Arnol'd, V.I. Small Denominators and the Stability Problem in Classical and Celestial Mechanics. Uspekhi Mat. Nauk 1963, 18, 91-192. [CrossRef]

16. Baker, H.F. An Introduction to the Theory of Multiply Periodic Functions; Cambridge University Press: Cambridge, UK, 1907.

17. Mumford, D. Tata Lectures on Theta I; Birkhäuser: Boston, MA, USA, 1982.

18. Mumford, D. Tata Lectures on Theta II; Birkhäuser: Boston, MA, USA, 1984.

19. Kotljarov, V.P.; Its, A.R. Explicit formulas for solutions of the Schrödinger nonlinear equation. Doklady. Akad. Nauk. Ukr SSSR. 1976, 11, 965-968. (In Ukranian)

20. Previato, E. Hyperelliptic Quasiperiodic and Soliton Solutions of the Nonlinear Schrödinger Equation. Duke Math. J. 1985, 52, 320-377. [CrossRef]

21. Tracy, E.R.; Chen, H.H. Nonlinear Self-modulation: An Exactly Solvable Model. Phys. Rev. A 1988, 37, 815-839. [CrossRef]

22. Bohl, P. Ueber die Darstellung von Funktionen einer Variabeln Durch Trigonometrische Reihen mit Mehreren einer Variablen Proportionalen Argumenten. Ph.D. Thesis, Universitat zu Dorpat, Dorpat, Estonia, 1893; 31p.

23. Bohr, H. Almost Periodic Functions; Chelsea Publishing Company: New York, NY, USA, 1947.

24. Besicovitch, A.S. Almost Periodic Functions; Cambridge University Press: Cambridge, UK, 1932.

25. Bochner, S. Beitraege zur Theorie der Fastperiodischen Funktionen, I. Teil: Funktionen Einer Variablen. Math. Ann. Bd. 1927, 96, 119-147. [CrossRef]

26. Bochner, S. Beitraege zur Theorie der Fastperiodischen Funktionen, II. Teil: Funktionen Mehrere Variablen. Math. Ann. Bd. 1927, 96, 383-409. [CrossRef]

27. Jessen, B. Bidrag Til Integralteorien for Funktioner af Uendelig Mange Variable; Habilitationsschrift: Kopenhagen, Denmark, 1930. 
28. Belokolos, E.D.; Bobenko, A.I.; Enol'skii, V.Z.; Its, A.R.; Matveev, V.B. Algebro-Geometric Approach to Nonlinear Integrable Equations; Springer: New York, NY, USA, 1994.

29. Eliasson, L.H.; Kuksin, S.B.; Marmi, S.; Yoccoz, J.-C. Dynamical Systems and Small Divisors; Springer: Berlin, Germany, 2002.

30. Dysthe, K.B. Note on a modification to the nonlinear Schrödinger equation for application to deep water waves. Proc. R. Soc. Lond. A 1979, 369, 105-114.

31. Trulsen, K.; Dysthe, K.B. A modified nonlinear Schrödinger equation for broader bandwidth gravity waves on deep water. Wave Motion 1996, 24, 281-289. [CrossRef]

32. Zakharov, V.E. Stability of periodic waves of finite amplitude on the surface of a deep fluid. J. Appl. Mech. Tech. Phys. USSR 1968, 2, 190. [CrossRef]

33. Zakharov, V.E.; Kuznetsov, E.A. Multi-scale expansions in the theory of systems integrable by the inverse scattering transform. In Solitons and Coherent Structures; Campbell, D.K., Newell, A.C., Schrieffer, R.J., Segur, H., Eds.; North-Holland: Amsterdam, The Netherlands, 1986; pp. 455-463.

34. Kimmoun, O.; Hsu, H.C.; Kibler, B.; Chabchoub, A. Nonconservative higher-order hydrodynamics modulation instability. Phys. Rev. 2017, 96, 022219. [CrossRef]

35. Barber, N.F.; Ursell, F.; Darbyshire, J.; Tucker, M.J. A frequency analyser used in the study of ocean waves. Nature Lond. 1946, 158, 329-332. [CrossRef]

36. Longuet-Higgins, M.S.; Barber, N.F. Four Theoretical Notes on the Estimation of Sea Conditions; Report N 1-N4 103.30/W; Admiralty Research Laboratory: Teddington, UK, 1946; (unpublished).

37. Longuet-Higgins, M.S. The directional spectrum of ocean waves and processes of wave generation. Proc. R. Soc. Lond. A 1962, 265, 286-315.

38. Longuet-Higgins, M.S. On the Nonlinear Transfer of Energy in the Peak of a Gravity-wave Spectrum: A Simplified Model. Proc. R. Soc. Long. A 1976, 347, 311-328.

39. Paley, R.C.; Weiner, N. Fourier Transform in the Complex Domain; American Mathematical Society Colloquium Publications: New York, NY, USA, 1934; Volume XIX.

40. Benjamin, T.B.; Feir, J.E. The disintegration of wave trains on deep water. Part 1. J. Fluid Mech. 1967, 27, 417430. [CrossRef]

41. Longuet-Higgins, M.S. The statistical analysis of a random, moving surface. Phil. Trans. Roy. Soc. Lond. 1957, 249, 321-387.

42. Doob, J.L. Stochastic Processes; John Wiley: New York, NY, USA, 1952.

43. Papoulis, A. Probability, Random Variables and Stochastic Processes; McGraw-Hill: New York, NY, USA, 1965.

44. Khintchine, A. Korrelationstheorie der stationaeren stochastischen Prozesse. Math. Ann. 1934, 109, 604-615. [CrossRef]

45. Rice, S.O. The mathematical analysis of random noise. Bell Syst. Tech. J. 1944, 23, 282-332. Bell Syst. Tech. J. 1945, 24, 46-156. [CrossRef]

46. Saffman, P.G.; Yuen, H.C. Stability of a plane soliton to infinitesimal two-dimensional perturbations. Phys. Fluids 1978, 8, 1450-1451. [CrossRef]

47. Yuen, H.C.; Lake, B.M. Nonlinear dynamics of deep-water gravity waves. Adv. Appl. Mech. 1982, 2, 67-229.

48. Hasimoto, H.; Ono, H. Nonlinear Modulation of Gravity Waves. J. Phys. Soc. Jpn. 1972, 33, 805-811. [CrossRef]

49. Mei, C.C. The Applied Dynamics of Ocean Surface Waves; John Wiley and Sons: New York, NY, USA, 1983.

50. Hasselmann, K. On the non-linear transfer in a gravity wave spectrum, Part 3. Evaluation of energy flux and sea-swell interactions for a Neumann spectrum. J. Fluid Mech. 1963, 15, 385-398. [CrossRef]

51. Phillips, O.M. On the generation of waves by turbulent wind. J. Fluid Mech. 1957, 2, 417-445. [CrossRef]

52. Young, I.R.; Verhagen, L.A.; Banner, M.L. A note on the bimodal directional spreading of fetch-limited wind waves. J. Geophys. Res. 1995, 100, 773-778. [CrossRef]

53. Ewans, K.C. Observations of the directional spectrum of fetch-limited waves. J. Phys. Oceanogr. 1998, 28, 495-512. [CrossRef]

54. Hwang, P.A.; Wang, D.W.; Walsh, E.J.; Krabill, W.B.; Swift, R.N. Airborne Measurements of the Wavenumber Spectra of Ocean Surface Waves. Part II: Direction Distribution. J. Phys. Ocean. 2000, 30, 2768-2787. [CrossRef]

55. Long, C.E.; Resio, D.T. Wind wave spectral observations in Currituck Sound, North Carolina. J. Geophys. Res. Ocean. 2007, 112, 1-21. [CrossRef] 
56. Komen, G.J.; Cavaleri, L.; Donelan, M.; Hasselmann, K.; Hasselmann, S.; Janssen, P.A.E.M. Dynamics and Modelling of Ocean Waves; Cambridge University Press: Cambridge, UK, 1994.

57. Chabchoub, A.; Mozumi, K.; Hoffmann, N.; Babanin, A.V.; Toffoli, A.; Steer, J.N.; van den Bremer, T.S.; Akhmediev, N.; Onorato, M.; Waseda, T. Directional soliton and breather beams. Proc. Nat. Acad. Sci. USA 2019, 116, 9759-9763. [CrossRef] [PubMed]

58. Matsutani, S. Hyperelliptic Solutions of KdV and KP equations: Reevaluation of Baker's Study on Hyperelliptic Sigma Functions. J. Phys. A Gen. Phys. 2000, 34, 4721. [CrossRef]

59. Kharif, C.; Pelinovsky, E.; Slunyaev, A. Rogue Waves in the Ocean; Springer: Berlin, Germany, 2008.

60. Whitham, G.B. Linear and Nonlinear Waves; John Wiley: New York, NY, USA, 1974.

61. Sutherland, P.; Melville, W.K. Field measurements and scaling of ocean surface wave-breaking statistics. Geophys. Res. Lett. 2015, 40, 3074-3079. [CrossRef]

62. Sutherland, P.; Melville, W.K. Measuring turbulent kinetic energy dissipation at a wavy sea surface. J. Atmos. Ocean. Technol. 2015, 32, 1498-1514. [CrossRef]

63. Sutherland, P.; Melville, W.K. Field measurement of surface and near-surface turbulence in the presence of breaking waves. J. Phys. Ocean. 2015, 45, 943-965. [CrossRef]

64. Cheng, H.-Y.; Chien, H. Implementation of S-band marine radar for surface wave measurement under precipitation. Remote Sens. Environ. 2017, 188, 85-94. [CrossRef]

65. Lund, B.; Collins, C.O.; Graber, H.C.; Terrill, E.; Herbers, T.H.C. Marine radar ocean wave retrieval's dependency on range and azimuth. Ocean Dyn. 2014, 64, 999-1018. [CrossRef]

66. Gudmestad, O.T. Modelling of waves for the Design of Offshore Structures. J. Mar. Sci. Eng. 2020, 8, 293. [CrossRef]

67. Fourier, J.J. Théorie Analytique de la Chaleur; Firmin Didot Père et Fils: Paris, France, 1822.

68. Zygmund, A. Trigonometric Series; Cambridge University Press: Cambridge, UK, 1932.

Publisher's Note: MDPI stays neutral with regard to jurisdictional claims in published maps and institutional affiliations. 